Florida International University FIU Digital Commons

$11-8-2016$

\title{
H I Structure and Kinematics of the Interstellar Medium in the LITTLE THINGS Galaxies
}

Nau R. Pokhrel

Florida International University, nauraj1@gmail.com

DOI: $10.25148 /$ etd.FIDC001205

Follow this and additional works at: https://digitalcommons.fiu.edu/etd

Part of the External Galaxies Commons, and the Stars, Interstellar Medium and the Galaxy Commons

\section{Recommended Citation}

Pokhrel, Nau R., "H I Structure and Kinematics of the Interstellar Medium in the LITTLE THINGS Galaxies" (2016). FIU Electronic Theses and Dissertations. 3028.

https://digitalcommons.fiu.edu/etd/3028

This work is brought to you for free and open access by the University Graduate School at FIU Digital Commons. It has been accepted for inclusion in FIU Electronic Theses and Dissertations by an authorized administrator of FIU Digital Commons. For more information, please contact dcc@fiu.edu. 


\section{FLORIDA INTERNATIONAL UNIVERSITY \\ Miami, Florida}

\section{H I STRUCTURE AND KINEMATICS OF THE INTERSTELLAR MEDIUM IN THE LITTLE THINGS GALAXIES}

A dissertation submitted in partial fulfillment of the

requirements of the degree of

DOCTOR OF PHILOSOPHY

in

PHYSICS

by

Nau Raj Pokhrel

2016 


\section{To: Dean Michael R. Heithaus}

College of Arts, Sciences and Education

This dissertation, written by Nau Raj Pokhrel, and entitled H i Structure and Kinematics of the Interstellar Medium in the LITTLE THINGS Galaxies, having been approved in respect to style and intellectual content, is referred to you for judgment.

We have read this dissertation and recommend that it be approved.

Grenville Draper

Walter Van Hamme

James Webb

Caroline Simpson, Major Professor

Date of Defense: November 8, 2016

The dissertation of Nau Raj Pokhrel is approved.

Dean Michael R. Heithaus College of Arts, Sciences and Education

Andrés G. Gil Vice President for Research and Economic Development and Dean of the University Graduate School

Florida International University, 2016 


\section{ACKNOWLEDGMENTS}

I would like to extend my sincere appreciation and gratitude to my advisor Dr. Caroline Simpson whose continuous inspiration, technical as well as scientific support and cooperation always motivated me in the entire research work. I got opportunity not only to learn the about research, but also many valuable suggestion in different aspects. To Dr. Simpson, I owe more then words for her immense caress and constant guidance throughout the time of entire work. I would like to express my sincere thanks my dissertation committee members, Dr. James Webb, Dr. Walter Van Hamme and Dr. Grenville Draper for the continuous help and support throughout the research. I would like to acknowledge Dr. Elias Brinks, University of Hertfordshire, UK, for his valuable suggestions and support for this work.

I am grateful to Department of Physics, FIU for accepting me as graduate student, and teaching assistant, and graduate program directors, Dr. Brian Raue and Dr. Jorge Rodriguez for their academic help and support. I am grateful to all professors and staff members of Physics Department for their support and help. I am really thankful to my senior friend and colleague Dr. Trisha Ashley and Dr. Gopal Bhatta for their help and advice in the beginning years of the research. My special thank to senior researcher and friend Dr. Ioannis Bagetakos for his constant help during the work.

I would like to thank LITTLE THINGS team for suggestions and support, NRAO for telescope time and public data access, and NSF AST-0707468 for financial support.

I am really grateful to my dear parents and my late grandmother for their continuous inspiration, encouragement and support in every difficult steps. At last but not the least, I am thankful to my wife Elina for her support throughout the dissertation years. 


\section{ABSTRACT OF THE DISSERTATION \\ H I STRUCTURE AND KINEMATICS OF THE INTERSTELLAR MEDIUM IN THE LITTLE THINGS GALAXIES}

by

Nau Raj Pokhrel

Florida International University, 2016

Miami, Florida

Professor Caroline Simpson, Major Professor

We present a catalog of the neutral atomic hydrogen structures (H I holes) and the analysis of their properties in nearby $(\leq 10.3 \mathrm{Mpc})$ gas-rich dwarf galaxies of the LITTLE THINGS (Local Irregulars That Trace Luminosity Extremes, The H I Nearby Galaxy Survey) group. We used high sensitivity $\left(\leq 1.1 \mathrm{mJy}^{\text {beam }}{ }^{-1}\right.$ channel $\left.^{-1}\right)$, high velocity resolution $\left(1.3 \mathrm{~km} \mathrm{~s}^{-1}\right.$ to $\left.2.6 \mathrm{~km} \mathrm{~s}^{-1}\right)$ and high linear resolution (average $\sim 110 \mathrm{pc}$; angular resolution range $\left.\sim 6^{\prime \prime}\right) \mathrm{H} \mathrm{I}$ data of 37 dwarf irregulars and four blue compact dwarf galaxies. We cataloged $\mathrm{H}$ I holes in the entire sample and studied the of the properties of holes. We also investigated the effect of $\mathrm{H}$ I porosity on star formation, and the correlation of the star formation rate (SFR) calculated from $\mathrm{H}$ I holes with standard star formation tracers $\mathrm{H} \alpha$ and FUV. We detected $306 \mathrm{H}$ I holes in LITTLE THINGS galaxies. We confirmed $22 \mathrm{kpc}$-sized holes, the largest and the smallest hole diameters are about $2.3 \mathrm{kpc}$ and $38 \mathrm{pc}$ (resolution limit) respectively. The expansion velocities of the holes range from $5 \mathrm{~km} \mathrm{~s}^{-1}$ (upper limit) to $30 \mathrm{~km} \mathrm{~s}^{-1}$, and the rotational velocities range from $6 \mathrm{~km} \mathrm{~s}^{-1}$ to $77 \mathrm{~km} \mathrm{~s}^{-1}$. The H I disk radii of the galaxies range from about $0.5 \mathrm{kpc}$ to $6.7 \mathrm{kpc}$. The kinetic ages of the holes range from about 1 to $127 \mathrm{Myr}$, and the estimated scale heights are varying from $61 \mathrm{pc}$ to $653 \mathrm{pc}$. The percentage distribution of the holes outside and inside the V-band break radius is nearly uniform, $49 \%$ and $51 \%$ respectively. In LITTLE THINGS galaxies, we found no obvious correlation between the surface and volume porosities, and SFR. 
However, two highest and two lowest porosity galaxies have no star formation at present. The holes are consistent with the SFR estimated from the energy required to create a hole and the star formation rates measured from $\mathrm{H} \alpha$ and FUV, indicating that the holes are consistent with a star formation origin. 


\section{TABLE OF CONTENTS}

1 Introduction . . . . . . . . . . . . . . . . . 1

1.1 Galaxies . . . . . . . . . . . . . . . . . . . . . 1

1.2 Dwarf Galaxies . . . . . . . . . . . . . . . . . . . . . 2

1.3 Dust and Gas in the ISM . . . . . . . . . . . . . . . . . 3

1.4 Neutral Hydrogen Observation . . . . . . . . . . . . . . . . 5

1.5 History of H I Structure Studies . . . . . . . . . . . . . . 6

1.6 The Role of Stellar Feedback in H i Structure Formation . . . . . . 7

1.7 Radio Interferometry . . . . . . . . . . . . . . . . . . . . . 8

1.8 Natural and Robust Weighting . . . . . . . . . . . . . . . . . 10

1.9 Moment Maps . . . . . . . . . . . . . . . . . . . . . . . . . . . . . 10

1.10 LITTLE THINGS and the Outline of This Project . . . . . . . 11

2 Identification of H I Structures . . . . . . . . . . . . . . . . . 13

2.1 Observation and Data Reduction . . . . . . . . . . . . . 13

2.2 Hole Detection Method . . . . . . . . . . . . . . . . . . . . . . . . 14

3 The Catalog . . . . . . . . . . . . . . . . . . . . . . . 23

3.1 Basic and Calculated Properties . . . . . . . . . . . . . . . 23

3.2 LITTLE THINGS Galaxies and Their Properties . . . . . . . . . 28

3.3 Analysis of the Properties . . . . . . . . . . . . . 106

4 Analysis . . . . . . . . . . . . . . . . . . . . . . . . 118

4.1 Porosity . . . . . . . . . . . . . . . . . . . . . . . 118

4.2 Star Formation Rate and Star Formation History . . . . . . . . . 122

5 Conclusions and Future Work . . . . . . . . . . . . . . 126

5.1 Conclusions . . . . . . . . . . . . . . . . . . 126

5.2 Future Work . . . . . . . . . . . . . . . . . . . . . . . . . . 128

\section{Bibliography}

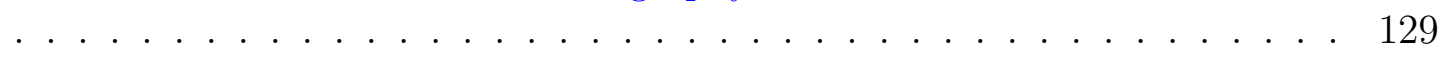

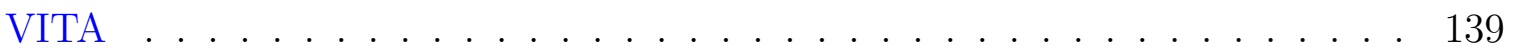




\section{LIST OF TABLES}

TABLE

PAGE

2.1 Basic Galaxy Information . . . . . . . . . . . . . . . . 15

3.1 Basic Properties of the Holes in the LITTLE THINGS Galaxies . . . 24

3.2 Hole Properties of CVnIdwA . . . . . . . . . . . . . . . . . 30

3.3 Hole Properties of DDO $43 \ldots \ldots \ldots \ldots$

3.4 Hole Properties of DDO $46 \ldots \ldots \ldots \ldots \ldots$

3.5 Hole Properties of DDO $47 \ldots \ldots \ldots \ldots$

3.6 Hole Properties of DDO $50 \ldots \ldots \ldots \ldots$

3.7 Hole Properties of DDO $52 \ldots \ldots \ldots \ldots$

3.8 Hole Properties of DDO $53 \ldots \ldots \ldots$

3.9 Hole Properties of DDO $63 \ldots \ldots \ldots \ldots \ldots$

3.10 Hole Properties of DDO $69 \ldots \ldots \ldots \ldots$

3.11 Hole Properties of DDO $70 \ldots \ldots \ldots \ldots$

3.12 Hole Properties of DDO $75 \ldots \ldots \ldots$

3.13 Hole Properties of DDO $87 \ldots \ldots \ldots \ldots$

3.14 Hole Properties of DDO $101 \ldots \ldots \ldots$

3.15 Hole Properties of DDO $126 \ldots \ldots \ldots \ldots \ldots$

3.16 Hole Properties of DDO $133 \ldots \ldots \ldots \ldots$

3.17 Hole Properties of DDO $154 \ldots \ldots \ldots$

3.18 Hole Properties of DDO $155 \ldots \ldots \ldots$

3.19 Hole Properties of DDO $165 \ldots \ldots$. . . . . . . . . . . . 64

3.20 Hole Properties of DDO $167 \ldots \ldots \ldots$

3.21 Hole Properties of DDO $168 \ldots \ldots \ldots$ 
3.22 Hole Properties of DDO $187 \ldots \ldots \ldots$

3.23 Hole Properties of DDO $210 \ldots \ldots \ldots$

3.24 Hole Properties of DDO $216 \ldots \ldots \ldots \ldots$

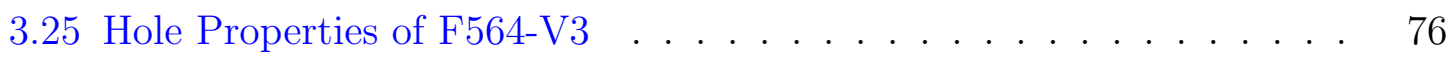

3.26 Hole Properties of Haro 29 . . . . . . . . . . . . . . . . . 78

3.27 Hole Properties of Haro $36 \ldots \ldots$

3.28 Hole Properties of IC $10 \ldots \ldots \ldots$

3.29 Hole Properties of IC $1613 \ldots \ldots \ldots$

3.30 Hole Properties of LGS $3 \ldots \ldots \ldots \ldots$

3.31 Hole Properties of M81dwA . . . . . . . . . . . . . . 88

3.32 Hole Properties of Mrk $178 \ldots \ldots \ldots$

3.33 Hole Properties of NGC 1569 . . . . . . . . . . . . . . . 92

3.34 Hole Properties of NGC 2366 . . . . . . . . . . . . . . . 94

3.35 Hole Properties of NGC $3738 \ldots \ldots \ldots$

3.36 Hole Properties of NGC $4214 \ldots \ldots \ldots$

3.37 Hole Properties of SagDIG . . . . . . . . . . . . . . . . . . 101

3.38 Hole Properties of UGC 8508 . . . . . . . . . . . . . . . . . 103

3.39 Hole Properties of WLM _ . . . . . . . . . . . . . . . . 105

4.1 Porosity and Star Formation Rate . . . . . . . . . . . . . 120 


\section{LIST OF FIGURES}

FIGURE

PAGE

2.1 Position-velocity diagram ( $p v$ slice; upper right) and intensity profile (bottom) of a hole of NGC 4214 . . . . . . . . . . . . . . . . .

$2.2 p v$ diagrams of three types of holes (These features are from NGC 4214). Top: Type 1 hole (completely blown out); Middle: Type 2 hole (partially blown out); and Bottom: Type 3 hole (intact). . . . . . . .

3.1 H I hole in the ISM of CVnIdwA (marked with ellipse). Blue ellipse indicates that it is Type 1 hole. . . . . . . . . . . . . . .

3.2 $\mathrm{H}$ I holes in the ISM of DDO 43 (marked with ellipses). Numbers inside the ellipse indicate the hole number from the catalog. Colors show the types of the holes: Blue ellipses are for Type 1, black are for Type 2, and red are for Type 3 holes. . . . . . . . . . . . . . . . .

3.3 H I holes in the ISM of DDO 46 (marked with ellipses). Numbers inside the ellipse indicate the hole number from the catalog. Colors show the types of the holes: Blue ellipses are for Type 1, black are for Type 2, and red are for Type 3 holes. . . . . . . . . . . . . . . . .

3.4 $\mathrm{H}$ I holes in the ISM of DDO 47 (marked with ellipses). Numbers inside the ellipse indicate the hole number from the catalog. Colors show the types of the holes: Blue ellipses are for Type 1, black are for Type 2, and red are for Type 3 holes. . . . . . . . . . . . . . . .

3.5 $\mathrm{H}$ I holes in the ISM of DDO 50 (marked with ellipses). Numbers inside the ellipse indicate the hole number from the catalog. Colors show the types of the holes: Blue ellipses are for Type 1, black are for Type 2, and red are for Type 3 holes. . . . . . . . . . . . . . . . . .

3.6 $\mathrm{H}$ I holes in the ISM of DDO 52 (marked with ellipses). Numbers inside the ellipse indicate the hole number from the catalog. Colors show the types of the holes: Blue ellipses are for Type 1, black are for Type 2, and red are for Type 3 holes. . . . . . . . . . . . . . . .

3.7 $\mathrm{H}$ I holes in the ISM of DDO 53 (marked with ellipses). Numbers inside the ellipse indicate the hole number from the catalog. Colors show the types of the holes: Blue ellipses are for Type 1, and red are for Type 3 holes. . . . . . . . . . . . . . . . . . . . . . 
3.8 H I holes in the ISM of DDO 63 (marked with ellipses). Numbers inside the ellipse indicate the hole number from the catalog. Colors show the types of the holes: Blue ellipses are for Type 1, black are for Type 2, and red are for Type 3 holes. . . . . . . . . . . . . . . . .

3.9 H I holes in the ISM of DDO 69 (marked with ellipses). Numbers inside the ellipse indicate the hole number from the catalog. Colors show the types of the holes: Blue ellipse is for Type 1, and black is for Type 2 hole. . . . . . . . . . . . . . . . . . . .

3.10 $\mathrm{H}$ I holes in the ISM of DDO 70 (marked with ellipses). Numbers inside the ellipse indicate the hole number from the catalog. Colors show the types of the holes: Blue ellipses are for Type 1, and black are for Type 2 holes. . . . . . . . . . . . . . . . . . . .

3.11 H I holes in the ISM of DDO 75 (marked with ellipses). Numbers inside the ellipse indicate the hole number from the catalog. Colors show the types of the holes: Blue ellipses are for Type 1 holes. . . . . . . . .

$3.12 \mathrm{H}$ I holes in the ISM of DDO 87 (marked with ellipses). Numbers inside the ellipse indicate the hole number from the catalog. Colors show the types of the holes: Blue ellipses are for Type 1, black are for Type 2, and red are for Type 3 holes. . . . . . . . . . . . . . . . . .

$3.13 \mathrm{H}$ I holes in the ISM of DDO 101 (marked with ellipses). Numbers inside the ellipse indicate the hole number from the catalog. Colors show the types of the holes: Black ellipse is for Type 2, and red is for Type 3 hole. . . . . . . . . . . . . . . . . . . . .

3.14 H I holes in the ISM of DDO 126 (marked with ellipses). Numbers inside the ellipse indicate the hole number from the catalog. Colors show the types of the holes: Blue ellipses are for Type 1, and red are for Type 3 holes. . . . . . . . . . . . . . . . . . . . . .

$3.15 \mathrm{H}$ I holes in the ISM of DDO 133 (marked with ellipses). Numbers inside the ellipse indicate the hole number from the catalog. Colors show the types of the holes: Blue ellipses are for Type 1 holes. . . . .

$3.16 \mathrm{H}$ I holes in the ISM of DDO 154 (marked with ellipses). Numbers inside the ellipse indicate the hole number from the catalog. Colors show the types of the holes: Blue ellipses are for Type 1, black are for Type 2, and red are for Type 3 holes. . . . . . . . . . . . . 
$3.17 \mathrm{H}$ I holes in the ISM of DDO 155 (marked with ellipses). Numbers inside the ellipse indicate the hole number from the catalog. Colors show the types of the holes: Blue ellipses are for Type 1 holes. . . . .

$3.18 \mathrm{H}$ I holes in the ISM of DDO 165 (marked with ellipses). Numbers inside the ellipse indicate the hole number from the catalog. Colors show the types of the holes: Blue ellipse is for Type 1, black is for Type 2, and red is for Type 3 hole. . . . . . . . . . . . . . . .

$3.19 \mathrm{H}$ I holes in the ISM of DDO 167 (marked with ellipses). Numbers inside the ellipse indicate the hole number from the catalog. Colors show the types of the holes: Blue ellipses are for Type 1, and red are for Type 3 holes. . . . . . . . . . . . . . . . . . . . . . .

$3.20 \mathrm{H}$ I holes in the ISM of DDO 168 (marked with ellipses). Numbers inside the ellipse indicate the hole number from the catalog. Colors show the types of the holes: Blue ellipse is for Type 1, and red is for Type 3 hole. . . . . . . . . . . . . . . . . . . .

$3.21 \mathrm{H}$ I holes in the ISM of DDO 187 (marked with ellipses). Numbers inside the ellipse indicate the hole number from the catalog. Colors show the types of the holes: Blue ellipses are for Type 1, and red are for Type 3 holes. . . . . . . . . . . . . . . . . . . . .

$3.22 \mathrm{H}$ I hole in the ISM of DDO 210 (marked with ellipse). Number inside the ellipse indicates the hole number from the catalog. Blue ellipse is for Type 1 hole. . . . . . . . . . . . . . . . . . . . .

$3.23 \mathrm{H}$ I holes in the ISM of DDO 216 (marked with ellipses). Numbers inside the ellipse indicate the hole number from the catalog. Colors show the types of the holes: Red ellipses are for Type 3 holes. . . . .

3.24 H I holes in the ISM of F564-V3 (marked with ellipses). Numbers inside the ellipse indicate the hole number from the catalog. Colors show the types of the holes: Black ellipses are for Type 2, and red are for Type 3 holes. . . . . . . . . . . . . . . . . . . . . . .

3.25 H I holes in the ISM of Haro 29 (marked with ellipses). Numbers inside the ellipse indicate the hole number from the catalog. Red ellipses are for Type 3 holes. . . . . . . . . . . . . . . . . . . . . . .

$3.26 \mathrm{H}$ I hole in the ISM of Haro 36 (marked with ellipse). Number inside the ellipse indicates the hole number from the catalog. Red ellipse is for Type 3 hole. . . . . . . . . . . . . . . . . . 
3.27 H I holes in the ISM of IC 10 (marked with ellipses). Numbers inside the ellipse indicate the hole number from the catalog. Colors show the types of the holes: Blue ellipses are for Type 1 holes. . . . . . . . .

$3.28 \mathrm{H}$ I holes in the ISM of IC 1613 (marked with ellipses). Numbers inside the ellipse indicate the hole number from the catalog. Colors show the types of the holes: Blue ellipses are for Type 1, black are for Type 2, and red are for Type 3 holes. . . . . . . . . . . . . . . . . .

3.29 H I holes in the ISM of LGS 3 (marked with ellipses). Numbers inside the ellipse indicate the hole number from the catalog. Colors show the types of the holes: Blue ellipses are for Type 1, and red are for Type 3 holes. . . . . . . . . . . . . . . . . . . .

3.30 $\mathrm{H}$ I holes in the ISM of M81dwA (marked with ellipses). Numbers inside the ellipse indicate the hole number from the catalog. Colors show the types of the holes: Blue ellipses are for Type 1, black are for Type 2, and red are for Type 3 holes. . . . . . . . . . . . . .

3.31 H I hole in the ISM of Mrk 178 (marked with ellipse). Number inside the ellipse indicates the hole number from the catalog. Black ellipse is for Type 2 hole. . . . . . . . . . . . . . . . . . . . . . .

3.32 H I holes in the ISM of NGC 1569 (marked with ellipses). Numbers inside the ellipse indicate the hole number from the catalog. Colors show the types of the holes: Blue ellipses are for Type 1, black are for Type 2, and red are for Type 3 holes. . . . . . . . . . . . . .

3.33 H I holes in the ISM of NGC 2366 (marked with ellipses). Numbers inside the ellipse indicate the hole number from the catalog. Colors show the types of the holes: Blue ellipses are for Type 1, black are for Type 2, and red are for Type 3 holes. . . . . . . . . . . . . . .

3.34 H I holes in the ISM of NGC 3738 (marked with ellipses). Numbers inside the ellipse indicate the hole number from the catalog. Colors show the types of the holes: Blue ellipses are for Type 1 holes. . . . .

$3.35 \mathrm{H}$ I holes in the ISM of NGC 4214 (marked with ellipses). Numbers inside the ellipse indicate the hole number from the catalog. Colors show the types of the holes: Blue ellipses are for Type 1, black are for Type 2, and red are for Type 3 holes. . . . . . . . . . . . . . . .

3.36 H I hole in the ISM of SagDIG (marked with ellipse). Number inside the ellipse indicates the hole number from the catalog. Blue ellipse is for Type 1 hole. 
3.37 H I holes in the ISM of UGC 8508 (marked with ellipses). Numbers inside the ellipse indicate the hole number from the catalog. Colors show the types of the holes: Blue ellipses are for Type 1, and red are for Type 3 holes. . . . . . . . . . . . . . . . . . . . . . . . . . . 102

$3.38 \mathrm{H}$ I holes in the ISM of WLM (marked with ellipses). Numbers inside the ellipse indicate the hole number from the catalog. Blue ellipses are for Type 1 holes. . . . . . . . . . . . . . . . . . . . . . . . . 104

3.39 Relative radial distribution of H I holes. . . . . . . . . . . . . . 107

3.40 Relative radial number distribution of $\mathrm{H}$ I holes for the entire sample. 107

3.41 Relative number distribution of the size of the $\mathrm{H}$ I holes. . . . . . . 108

3.42 Relative number distribution of the size for the $\mathrm{H}$ I holes of entire sample.109

3.43 Distribution of H I holes: diameter vs. normalized V-band scale length. 110

3.44 Relative number distribution of the expansion velocity of Type 2 and Type 3 holes. . . . . . . . . . . . . . . . . . . . . . . . 111

3.45 Relative number distribution of the expansion velocity of Type 2 and Type 3 holes for the entire sample. . . . . . . . . . . . . . . . . 112

3.46 Distribution of H I holes: Expansion velocities with respect to the location. Blue colored points are the upper limits of expansion velocities for Type 1 holes, black and red are the expansion velocities of Type 2 and Type 3 holes respectively. . . . . . . . . . . . . . . . . . . 112

3.47 Distribution of the kinetic ages of H I holes. . . . . . . . . . 113

3.48 Distribution of $\mathrm{H}$ I holes per unit area inside and outside of the break radius. . . . . . . . . . . . . . . . . . . . . . . 114

3.49 Surface brightness profiles and radial color distributions for DDO 75. The dotted black line is the best exponential fit to the V-band data (Herrmann et al. 2013). . . . . . . . . . . . . . . . . . . . . . 114

3.50 Relative number distribution of the estimated energy required to form Hi holes. . . . . . . . . . . . . . . . . . . . . . . 115

3.51 Estimated energy required to form $\mathrm{H}$ I holes of the entire sample with respect to their radial distributions. . . . . . . . . . . . . . . 116 
3.52 Estimated energy required to form $\mathrm{H}$ I holes vs. their normalized radial locations. . . . . . . . . . . . . . . . . . . . 117

4.1 Porosity vs. star formation rate: a) surface porosity vs. the SFR calculated from $\mathrm{H} \alpha$ emission, b) volume porosity vs. the SFR calculated from $\mathrm{H} \alpha$ emission, c) surface porosity vs. the SFR calculated from FUV emission, and d) volume porosity vs. the SFR calculated from FUV emission. In Figures a and b, there is no star formation in the bottom four galaxies; SFR $=-7$ is assigned just for plotting purposes. 121

4.2 Kinetic age of the $\mathrm{H}$ I holes vs. the number of supernovae required to form them. . . . . . . . . . . . . . . . . . . . . . . . 123

4.3 The star formation rate estimated from the energy required to form the $\mathrm{H}$ I holes vs. the stellar star formation rates $\mathrm{H} \alpha$ (left) and FUV (right). The solid line is the best fit linear regression line. The shaded region shows the confidence limits (95\%) for the expected value. . . 


\section{CHAPTER 1}

\section{Introduction}

\section{Chapter Overview}

We present a brief introduction of dwarf galaxies and their interstellar medium (ISM). We also provide a short history of neutral hydrogen ( $\mathrm{HI}$ ) holes and shells, and summarize interferometry, moment maps, and the outline of this dissertation.

\subsection{Galaxies}

Since the discovery of galaxies by Edwin Hubble [1], there has been a lot of progress in the study of galaxy formation and evolution. Nowadays, many nearby and distant galaxies are being studied in all possible electromagnetic spectrum ranges. The study of galaxies to the present day has provided a lot of information about their origin and the evolution of the universe.

The ISM of galaxies is inhomogeneous and comprises about $10 \%$ of their total baryonic mass on average [2]. In the present research, we are interested in studying the interstellar H I gas and structures in the ISM of nearby dwarf galaxies to examine their relationship with star formation and stellar feedback. We can study high resolution maps, observe the structures in the ISM, and locate the position of star clusters and star-forming regions. Studying the ISM and star formation-gas interaction is important because it helps to understand the evolution of galaxies. 


\subsection{Dwarf Galaxies}

Dwarf galaxies are the most abundant and dynamically the simplest galaxy systems in the universe, having a wide range of morphology and physical properties. Dwarfs are also low mass, ranging in mass from $10^{6}$ to $10^{1} 0 M_{\odot}$, which is about $10^{3}$ to $10^{5}$ times less than the ellipticals or spirals $[3,4]$. They have shallower gravitational potential and thicker disks than the spirals. They are small in size (1 to $10 \mathrm{kpc}$ in diameter) and have low luminosities ranging from $-5 \gtrsim M_{\mathrm{B}} \gtrsim-18$, because of their low stellar masses $[5,6]$. These galaxies rotate with low rotational speed $\left(10\right.$ to $\left.100 \mathrm{~km} \mathrm{~s}^{-1}\right)$ and possess solid body rotation.

\subsubsection{Why Study Dwarfs?}

Dwarf galaxies are believed to be the earliest-forming galaxies and the building blocks for the large systems $[7,8]$. By studying dwarf galaxies, we can get a better picture of the evolution of galaxies. [9].

Among dwarfs, we are interested in dwarf irregulars because of their primitive structure, and their role in galaxy formation. Although dwarf irregular galaxies have little dust and low metallicity even in their central regions, they are rich in neutral atomic hydrogen gas in comparison to spirals [10,11], and hence are the ideal laboratories to study the star-gas interaction in star formation, the evolution of galaxies, and hence the evolution of the early universe [3,12-18].

The critical gas density of any galaxy is the column density of galactic disk gas above which the disk becomes unstable and can collapse to form stars [19]. In irregular galaxies, the ratio of the observed gas density to the critical gas density is two to three times less than that of spirals in the inner region as well as beyond

the outer radius where star formation apparently ends [11]. Studies of the ionized 
hydrogen gas (H II) in dwarfs shows that dwarf galaxies have star-forming regions as large as those in spirals and they are forming stars not only in the densest inner region, but also in the far outer parts $[11,20]$. It is important to understand how the H I gas collapses and forms stars in such simple systems to understand the evolution of galaxies. Moreover, the gas accretion mechanism [21,22], and the role of ionized gas and re-ionization on star formation can be understood from dwarfs [23-27].

\subsection{Dust and Gas in the ISM}

The interstellar medium (ISM) contributes roughly $10 \%$ to $15 \%$ of the total baryonic mass of galaxies. A huge portion $(\approx 99 \%)$ of the total baryonic mass of the ISM contains gas. The remaining $1 \%$ are the dust grains [2].

\subsubsection{Dust}

Dust particles are usually found in dark molecular clouds formed by stellar radiation or supernovae explosions. They mainly consist of oxygen, magnesium, silicon, iron and their compounds like silicates, oxides, ice particles, and much of the carbonbased molecules. Although dust is about $0.1 \%$ of total baryonic mass and about $1 \%$ in comparison to gas mass, it absorbs a large fraction $(30-50 \%)$ of stellar radiation [2]. Dust plays a significant role in forming molecular hydrogen and other complex molecules through catalysis $[2,28]$. Moreover, dusty molecular clouds promote star formation.

\subsubsection{Gas}

The existence of gas clouds in the ISM was first suggested by Johannes Hartmann in 1904 from the study of emission and absorption spectra of binary stars such as $\delta$ 
Orionis [29]. He found that the Doppler shift of some spectra were not as expected, but could be explained by the existence of gas clouds between the stars and the observation point. Those lines were emitted from ionized gas clouds due to stellar radiation. From the analysis of $\mathrm{V}$ band and UV absorption spectra, nearly all the elements below the atomic number of Zinc $(30)$ have been observed. Hydrogen $(\approx 70 \%)$ and helium $(\approx 30 \%)$ dominate their composition, which is similar to stellar compositions [2]. The distribution of gases in the ISM is inhomogeneous and dynamic. The composition of ions, atoms and molecules, the pressure, temperature and density of different regions in the ISM are constantly changing due to factors such as stellar radiation, supernovae explosions, and thermal and gravitational instabilities.

The gas in the ISM may be either in a neutral (H I) or ionized (H II) state. Neutral ISM may also have subgroups such as molecular medium, cold neutral (atomic) medium, and hot (atomic) medium [30]. The molecular medium is a cold $(<100 \mathrm{~K})$ neutral medium which contains molecular hydrogen, and is very difficult to observe because of its very weak radiation. Therefore, a carbon monoxide tracer $(\lambda=2.6$ $\mathrm{mm}$ ) is usually used to detect the molecular gas (the $\mathrm{H}_{2}$ to $\mathrm{CO}$ ratio is about $10^{4}$ to $\left.10^{5}\right)$. The volume filling factor of $\mathrm{H}_{2}$ is less than a percent with an average volume density of about $10^{3}$ atoms $\mathrm{cm}^{-3}[2]$. The mass and the size of typical clouds are about $10^{5} M_{\odot}$ and 40 pc respectively [31].

The cold neutral medium contains atomic hydrogen. A regular cold H I cloud is about $10 \mathrm{pc}$ in size and has mass $\approx 50 M_{\odot}$. On average, the temperature of a cold $\mathrm{H}$ I region is about $100 \mathrm{~K}$ and the volume filling factor is $\approx 2-4 \%$ with a volume density of $\approx 10$ to 100 atoms $\mathrm{cm}^{-3}[2,32]$. The warm neutral medium also contains atomic hydrogen and comprises about half of the total $\mathrm{H}$ I with a volume filling factor $\geq 30 \%$, but the density of the gas is low with $\approx 0.3$ atoms $\mathrm{cm}^{-3}$ and a temperature 
about $6000 \mathrm{~K}[31,33]$. Both $\mathrm{H}$ I gas regions emit $21 \mathrm{~cm}$ hydrogen radiation and can be observed with radio telescopes.

Similarly, the ionized ISM can be studied in both warm and hot ionized phases [30]. The warm ionized medium is believed to be formed mostly from the stellar radiation of $\mathrm{O}$ and $\mathrm{B}$ stars, and in some cases, from the collision of the gas. It is associated with $\mathrm{H}$ II regions and can be traced by $\mathrm{H} \alpha$ emission. Its volume filling factor is $\approx 15 \%$ with density about 10 to $10^{6}$ electrons $\mathrm{cm}^{-3}$ and temperature about $8000 \mathrm{~K}$ [32]. The hot ionized ISM is usually formed from supernovae explosions, and can be detected by UV and X-ray observation. Its volume density is about $10^{-3}$ electrons $\mathrm{cm}^{-3}$ with filling factor nearly $50 \%$ and temperature range $\approx 2 \times 10^{5} \mathrm{~K}[2]$.

\subsection{Neutral Hydrogen Observation}

Neutral hydrogen consists of an electron and the nucleus with a proton. When there is a hyperfine transition of the ground state of neutral hydrogen, i.e., when the electron spin and the nuclear spin of the hydrogen atom change from the parallel to antiparallel state, electromagnetic radiation of frequency $1420.4 \mathrm{MHz}(\lambda=21.1 \mathrm{~cm})$ and energy $\approx$ $10^{-5} \mathrm{eV}$ is emitted, which is known as $21 \mathrm{~cm}$ radiation. This theory was first proposed by Hendrik van de Hulst in 1944, and was verified observationally by different scientific teams such as Ewen and Purcell [34], Muller and Oort [35], and others.

However, the history of $\mathrm{H}$ I observations began about a decade before, with Karl Jansky. He first detected radio waves from the space in 1932 [36,37]. The firstever radio sky map was developed by Grote Reber in 1940 [38]. Since then, many discoveries have been made in the field of radio astronomy.

There is a very low probability $\left(2.87 \times 10^{-15} \mathrm{~s}^{-1}\right)$ of a neutral hydrogen atom going through a spin-flip transition, i.e. its half life is $\approx 1.11 \times 10^{7} \mathrm{yr}$ [39]. However, H I observation is made possible because of the large amount of hydrogen in the ISM 
of galaxies. In most galaxies, the H I disk is much larger than the optical disk. The H I observations are very useful to study the gas content, morphology and kinematics of galaxies. The $\mathrm{H}$ is also interrelated with star formation and hence the dynamics and evolution of galaxies.

\subsection{History of H I Structure Studies}

Neutral hydrogen structures were first found by Westerlund and Mathewson [40] in the ISM of the Large Magellanic Cloud, followed by Hindman [41] in the Small Magellanic Cloud, who suggested that the cause of such structures might be "super supernovae.' Following the survey by Weaver and Williams [42], Heiles [43] discovered expanding $\mathrm{H}$ I holes in the Milky Way as expected in the theoretical explanation by Cox and Smith [44], and McKee and Ostriker [45]. Later, Heiles [46, 47] and $\mathrm{Hu}[48]$ prepared catalogs of expanding H I holes in the Milky Way. Later studies by Brinks and Bajaja [49], Puche et al. [50], Kim et al. [51], Walter and Brinks [52], Chu [53], Warren et al. [54] and many others detected or/and analyzed the possible origins and properties of H I shells, holes, or bubble-like structures. Some galaxies have an ISM that is dominated by such structures, which might have been formed by a single or a combination of various processes such as stellar feedback, i.e., stellar winds and supernovae (SNe) explosions [18,55-61], from turbulence in the ISM, i.e., the motion of high velocity gas [58,62-65], a high-energy gamma-ray burst [66-68], or thermal and gravitational instabilities [69]. Relaño et al. [70] proposed that H i holes are originated from H II regions. The explanation of multiple supernovae explosions was supported by Weisz et al. [18] while studying the Hi holes in Holmberg II. The numerical simulation of Dib and Burkert [69] suggests that the H I holes in dwarf irregulars can form as a combined result of turbulence, thermal instabilities, and gravitational instabilities whereas the simulation of Vorobyov and Basu [71] shows 
that stellar feedback (multiple SNe or a hypernova explosion associated with gamma ray burst, and the vertical impact of an high velocity cloud) describes the formation of $\mathrm{H}$ I holes with more accuracy than the other processes [54].

\subsection{The Role of Stellar Feedback in H I Structure Formation}

The energy estimate required to form $\mathrm{H}$ I holes ranges from $10^{50}$ to $10^{54} \mathrm{erg}$, which is derived from the measured expansion velocities $\left(v_{\exp }\right)$. Although observations show that holes are smaller than predicted by the estimation of adiabatic evolution, stellar feedback is still considered to play an important role in $\mathrm{H}$ I structure formation because

this theory is based on shock-heated $\left(\geq 10^{6} \mathrm{~K}\right)$ pressure. It has some uncertainty such as growth rate discrepancy which may be due to the inhomogeneity of the ISM, energy transfer from the holes to cosmic rays, overestimation of input energy, and/or underestimation of ambient density/pressure [72-74].

The comparison of $21 \mathrm{~cm} \mathrm{H}$ I observations with those at other wavelengths provides a better understanding of hole formation, gas dynamics, and the relation between structures in the HI and stellar feedback. Some works of the comparison of $\mathrm{HI}$ and $\mathrm{H} \alpha$ images are in Kennicutt et al. [75], Martin [76], Walter et al. [77], Pidopryhora et al. [78]; and wavelet analyses are in Frick et al. [79], Huhges et al. [80], Tabatabaei et al. [81] and Dumas et al. [82]. Currently, infrared images (older stellar populations) from the Spitzer Space Telescope and ultraviolet images (younger starforming regions) from the Galaxy Evolution Explorer (GALEX) satellite telescope are the most widely used to compare with H I maps.

In the comparison of images at different wavelengths, we expect that the smaller and the younger holes might also have $\mathrm{H} \alpha$ emission from active star formation regions, whereas the shells of the older and the larger holes might be filled with $\mathrm{O}$ and B stars emitting UV radiation. For more metal-rich galaxies (such as spirals), we also expect 
$\mathrm{CO}$ emission from the outer rims of the expanding holes where molecular clouds are being compressed $[2,83]$.

\subsection{Radio Interferometry}

For radio-wavelength observations we need large telescopes to achieve high resolution. As the wavelength of the radiation increases, we need larger and larger telescopes because the resolution angle is inversely related to the diameter of the collecting surface. As an example, to get $1^{\prime \prime}$ angular resolution at a wavelength of $1 \mathrm{~m}$, we need an antenna about $200 \mathrm{~km}$ in diameter! Therefore, interferometers are used for longer wavelength observations. In radio interferometry, large antennae are used to receive and superimpose the waves so that the system works as single telescope with a diameter equivalent to the length of the longest baseline (distance between any two antennae pair). The interferometry reduces many engineering problems involved in constructing a very large single dish for high resolution. Our data are from the Very Large Array (VLA) interferometer, a National Radio Astronomy Observatory facility ${ }^{1}$. We present some brief information about the VLA.

The VLA interferometer has 27 antennae, each $25 \mathrm{~m}$ in diameter, placed in Y-shaped configuration in New Mexico. The array is usually placed in one of four principal configurations, categorized as A, B, C and D, with gradual decreasing order in resolution and increasing order in sensitivity. For H I observations, the maximum possible baseline lengths of A, B, C and D arrays are $36 \mathrm{~km}, 11 \mathrm{~km}, 4 \mathrm{~km}$ and $1 \mathrm{~km}$ respectively.

The lowest possible resolution limit of the radio telescope is given by beam size or beam width (measured in arcsec). Usually, resolution is calculated in terms of half power beam width $(\mathrm{HPBW} \approx \lambda / \mathrm{d})$. It is the angular gap in which the power of the

\footnotetext{
${ }^{1}$ The National Radio Astronomy Observatory is a facility of the National Science Foundation operated under cooperative agreement by Associated Universities, Inc.
} 
main lobe decreases by half $(-3 \mathrm{~dB})$ or the radiation decreases by $50 \%$, where $d$ is the diameter of the telescope dish and $\lambda=21 \mathrm{~cm}$ for $\mathrm{H}$ I radiation. The nominal beam width of A configuration is $1.3^{\prime \prime}$ with $36^{\prime \prime}$ maximum detectable structure scales whereas B array can detect size scales up to $120^{\prime \prime}$ with beam width of $4.3^{\prime \prime}$. The largest detectable size for $\mathrm{C}$ and $\mathrm{D}$ arrays is $970^{\prime \prime}$ with beam width of $14^{\prime \prime}$ and $46^{\prime \prime}$ respectively. Therefore, the D configuration of has the lowest resolution but the highest sensitivity limit.

For a particular source, its sky brightness distribution is given by the response $(R)$ of the interferometer, where $R$ is calculated as:

$$
R(u, v)=\iint S(x, y) e^{-2 \pi i(u x+v y)} d a .
$$

Here, $a=a(x, y)$ denotes the plane of the image in orthogonal coordinates, $(u, v)$ denotes the aperture domain in Fourier space, and $S$ is the intensity distribution of the image. We can obtain the intensity distribution by calculating the inverse Fourier Transform of brightness distribution:

$$
S_{\mathrm{D}}(x, y)=\iint R(u, v) e^{2 \pi i(u x+v y)} d u d v
$$

but the equation converts the phase information into the "dirty" image $S_{\mathrm{D}}$. The dirty image is separated into a true image and the synthesized dirty beam by using the convolution theorem

$$
S_{\mathrm{D}}(x, y)=S(x, y) B(x, y)
$$

where $S(x, y)$ is the flux or the desired image distribution, and

$$
B(x, y)=\iint S(u, v) e^{2 \pi i(u x+v y)} d u d v
$$


is the Fourier transform of the sampling function, i.e., the dirty beam. From $S(x, y)$, data cubes are created in position-velocity space. Data cubes are three dimensional representations of the flux mapped as the $\mathrm{H}$ I distribution in individual velocity channels. Usually, right ascension and declination are taken as the $x$ and $y$ axes and velocity as the third dimension.

\subsection{Natural and Robust Weighting}

By adjusting the beam size, i.e., adjusting the weighting of data from different baseline lengths, H I data can be mapped at different sensitivities and resolutions. Natural and robust weighting methods were used to produce images for this project.

Natural weighting is used to produce images with higher sensitivity. However, such images have poor resolution because the (more numerous) data from shorter baselines (lower resolution) are more heavily weighted than the (scanter) data from longer baselines.

Robust weighting allows the user to adjust the weighting of data from different baseline lengths. By more heavily weighting data from longer baselines, images with higher resolution but lower sensitivity (due to increased noise) are produced.

\subsection{Moment Maps}

Moment maps are made by integrating the $\mathrm{H}$ I data cubes along the velocity channels. Moment 0 maps are the flux density maps, which show the $\mathrm{H}$ I distribution in the two dimensional plane of the sky, and are given by

$$
\operatorname{Mom}_{0}(x, y)=\int S(x, y, v) d v=S(x, y) .
$$


The integrated flux density and the major and minor axes $\left(\theta_{\operatorname{maj}}, \theta_{\min }\right)$ of the synthesized beam are used to calculate brightness temperature $\left(T_{\mathrm{B}}\right)$ as:

$$
T_{\mathrm{B}}(K)=\frac{S \lambda^{2}\left(\mathrm{Jy} \mathrm{beam}^{-1} \mathrm{~cm}^{2}\right)}{2.7 \theta_{\text {maj }} \theta_{\text {min }}(\mathrm{sq} \cdot \operatorname{arcmin})}
$$

where $1 \mathrm{Jy}=10^{-26} \mathrm{~W} \mathrm{~m}^{-2} \mathrm{~Hz} . T_{\mathrm{B}}$ is directly related to the $\mathrm{H}$ I column density:

$$
\left.N_{\mathrm{HI}}(x, y)(\operatorname{atoms~cm})^{-2}\right)=1.82 \times 10^{18} \int T_{\mathrm{B}}(x, y, v) d v .
$$

From the flux density and the distance to the source $(D)$, we can calculate the H I mass content $\left(M_{\mathrm{H}_{\mathrm{I}}}\right)$ in the galaxy as:

$$
\frac{M_{\mathrm{HI}}}{M_{\odot}}=\left(2.35 \times 10^{5}\right)\left(\frac{D^{2}}{\mathrm{Mpc}^{2}}\right) \int_{v}\left(\frac{S(v) d v}{\mathrm{Jy} \mathrm{km} \mathrm{s}^{-1}}\right) .
$$

Moment 1 maps are the velocity maps, i.e. the flux-weighted mean velocity distribution given by

$$
\operatorname{Mom}_{1}(x, y)=\frac{\int S(x, y, v) v d v}{M_{0}(x, y)}
$$

and the Moment 2 maps are the velocity dispersion maps given by

$$
\operatorname{Mom}_{2}(x, y)=\sqrt{\frac{\int S(x, y, v)\left(v-M o m_{1}(x, y)\right)^{2} d v}{\operatorname{Mom}_{0}(x, y)}} .
$$

\subsection{LITTLE THINGS and the Outline of This Project}

The research described in this dissertation is a part of the LITTLE THINGS ${ }^{2}$ (Local Irregulars That Trace Luminosity Extremes, The H I Nearby Galaxy Survey) project. LITTLE THINGS studies local dwarf irregular galaxies to understand the general idea

\footnotetext{
${ }^{2}$ https://science.nrao.edu/science/surveys/littlethings
} 
of star formation. In particular, it examines the cause of triggering and the regulation of star formation in simple systems where established theories of star formation do not work. The project also studies the outer disks of the galaxies where the gas is highly dispersed and gravitationally stable but star formation is ongoing. In addition, the project also studies starburst in blue compact dwarfs.

The purpose of this dissertation research is to study holes in the ISM, porosity, and their relation to star formation in nearby dwarf galaxies. In this project, we study the kinematics of $\mathrm{H}$ I holes/shells in dwarf galaxies, investigate the effect of porosity on star formation, and examine whether the observed H I sructures are likely to be related to star-formation activity. The LITTLE THINGS H I observations are explained in Chapter 2. More detail about the observation and calibration of the $\mathrm{H} \mathrm{I}$ data is described in Hunter et al. [84].

The next step is to study the observed and calculated properties of the holes which we present in Chapter 3. In the same Chapter, the inter-relation among the properties of the holes and subsequent analysis is presented. We are looking for, 'Is there any relation between the H I structures and the star formation?' which is explained in Chapter 4. Conclusions and future work wrap up the dissertation. 


\section{CHAPTER 2}

\section{Identification of H I Structures}

\subsection{Observation and Data Reduction}

The LITTLE THINGS sample contains 41 gas-rich, non-interacting, relatively isolated nearby dwarf galaxies with a wide range of properties. Among them, 37 are dwarf irregulars (dIrrs) and the rest are blue compact dwarfs (BCDs), as listed in Table 2.1. The average distance of LITTLE THINGS galaxies is $3.7 \mathrm{Mpc}$, with the nearest and the farthest galaxies, NGC 6822 and DDO 52, at distances of $0.5 \mathrm{Mpc}$ and 10.3 Mpc respectively. DDO 63 and NGC 3738 are two face-on galaxies in the sample. The project studies the high sensitivity $\left(\leq 1.1 \mathrm{mJy}^{\mathrm{beam}}{ }^{-1}\right.$ channel $\left.^{-1}\right)$, high angular resolution $\left(\approx 6^{\prime \prime}\right)$, and high velocity resolution $\mathrm{H}$ I data of the sample. The H I data for 20 galaxies are taken from the VLA archives, with $2.6 \mathrm{~km} \mathrm{~s}^{-1}$ channel separation; the separation is $1.3 \mathrm{~km} \mathrm{~s}^{-1}$ for the new data obtained from the VLA in the B, C and D array configurations by the LITTLE THINGS group. The angular resolution limit of $6^{\prime \prime}$ corresponds to linear resolutions ranging from $\approx 26$ to $300 \mathrm{pc}$ with 110 pc at the average space distance at $3.7 \mathrm{Mpc}$. The V band disk scale length $R_{\mathrm{D}}$ ranges from $0.17 \mathrm{kpc}$ for DDO 210 to $2.26 \mathrm{kpc}$ for DDO 165 .

\subsubsection{AIPS}

The $\mathrm{NRAO}^{1}$ Astronomical Image Processing System (AIPS) is a large (over a million lines of code) interactive software package which has many subprograms to calibrate, map, edit and analyze radio interferometric data. It was designed in 1978 mainly for VLA data, and has about 400 application tasks.

\footnotetext{
${ }^{1}$ The National Radio Astronomy Observatory is a facility of the National Science Foundation operated under cooperative agreement by Associated Universities, Inc.
} 
Astronomical Image Processing System was used to calibrate and map the observed data. Specifically, LITTLE THINGS used standardized algorithmic 'recipes' for calibration. Multi-Scale (M-S) CLEAN was used for mapping. In the first mapping step, natural-weighted and robust-weighted H I data cubes were made using the task IMAGR in AIPS. Here, two of the dimensions of the cube are the spatial coordinates and velocity is the third dimension. In the second step, with the help of task XMOM, each data cube was integrated along the velocity axis to make two-dimensional integrated H I flux density (Moment 0) maps, H I velocity field (Moment 1) maps, and H I velocity dispersion (Moment 2) maps. The data reduction and conversion to the images are described in detail in Hunter et al. [84]. Natural-weighted flux density maps and data cubes are used to find the structures (holes, shells etc.) in the sample, to study low density gas around those structures, and their relation with star formation. If they are not detailed enough, robust-weighted maps and data cubes are helpful in such cases. We also used tasks BLANK and ISPEC to calculate the total area covered by the $\mathrm{H}$ I mass out to $5 \times 10^{19}$ atoms $\mathrm{cm}^{-2}$.

Data of other wavelengths are used to get information about the population of stars of different ages. The FUV data is from GALEX, $\mathrm{H} \alpha$ images are from Spitzer, UBV and $\mathrm{H} \alpha$ data and images are from Hunter and Elmegreen $[85,86]$.

\subsection{Hole Detection Method}

Both automated detection algorithms and visual inspection methods are used by astronomers to detect $\mathrm{H}$ I holes in galaxies. Thilker et al. [87] used expanding models to detect holes in NCG 2403 whereas Mashchenko et al. [88] used 3D hydrodynamical simulations to discover holes in the same galaxy. Daigle et al. [89] used a neural network algorithm based on velocity spectra to identify such structures. Later on, Ehlerova and Palous [90,91] discovered holes in the Milky Way by using a hole- 
Table 2.1: Basic Galaxy Information

\begin{tabular}{|c|c|c|c|c|c|c|}
\hline Galaxies & $\begin{array}{c}\text { RA (J2000) } \\
\text { (hh mm ss.s) }\end{array}$ & $\begin{array}{c}\text { Dec (J2000) } \\
\text { (dd mm ss) }\end{array}$ & $\begin{array}{l}\text { Distance }^{\mathrm{a}} \\
(\mathrm{Mpc})\end{array}$ & $\begin{array}{r}\mathrm{PA}^{\mathrm{a}} \\
(\mathrm{deg})\end{array}$ & $\begin{array}{l}\text { Inclination }{ }^{\mathrm{a}} \\
\text { (deg) }\end{array}$ & $\begin{array}{r}R_{\mathrm{D}}{ }^{\mathrm{b}} \\
(\mathrm{kpc})\end{array}$ \\
\hline CVnIdwA & $\begin{array}{lll}12 & 38 & 40.2\end{array}$ & $+3245 \quad 40$ & 3.6 & 80 & 41.0 & 0.57 \\
\hline DDO 43 & $\begin{array}{lll}07 & 28 & 17.8\end{array}$ & $+40 \quad 46 \quad 13$ & 7.8 & 6.5 & 48.5 & 0.41 \\
\hline DDO 46 & $\begin{array}{lll}07 & 41 & 26.6\end{array}$ & +400639 & 6.1 & 84 & 28.6 & 1.14 \\
\hline DDO 47 & $\begin{array}{lll}07 & 41 & 55.3\end{array}$ & $+1648 \quad 08$ & 5.2 & -70 & 64.4 & 1.36 \\
\hline DDO 50 & $\begin{array}{lll}08 & 19 & 08.7\end{array}$ & +704325 & 3.4 & 18 & 46.7 & 1.10 \\
\hline DDO 52 & $\begin{array}{lll}08 & 28 & 28.5\end{array}$ & +415121 & 10.3 & 5 & 51.1 & 1.32 \\
\hline DDO 53 & $\begin{array}{lll}08 & 34 & 08.0\end{array}$ & $\begin{array}{lll}+66 & 10 & 37\end{array}$ & 3.6 & 81 & 64.4 & 0.72 \\
\hline DDO 63 & $\begin{array}{lll}09 & 40 & 30.4\end{array}$ & $+71 \quad 11 \quad 02$ & 3.9 & 0 & 0 & 0.68 \\
\hline DDO 69 & $\begin{array}{lll}09 & 59 & 25.0\end{array}$ & $+30 \quad 44 \quad 42$ & 0.8 & -64 & 60.3 & 0.19 \\
\hline DDO 70 & $\begin{array}{lll}10 & 00 & 00.9\end{array}$ & +051950 & 1.3 & 88 & 57.8 & 0.48 \\
\hline DDO 75 & $\begin{array}{lll}10 & 10 & 59.2\end{array}$ & -044156 & 1.3 & 41 & 33.5 & 0.22 \\
\hline DDO 87 & $\begin{array}{lll}10 & 49 & 34.7\end{array}$ & +653146 & 7.7 & 76.5 & 58.6 & 1.31 \\
\hline DDO 101 & $1155 \quad 39.4$ & +313108 & 6.4 & -69 & 49.4 & 0.93 \\
\hline DDO 126 & $12 \quad 27 \quad 06.5$ & +370823 & 4.9 & -41 & 67.7 & 0.87 \\
\hline DDO 133 & $12 \quad 3255.4$ & +313214 & 3.5 & -6 & 49.4 & 1.24 \\
\hline DDO 154 & $12 \quad 54 \quad 06.2$ & +270902 & 3.7 & 46 & 65.2 & 0.59 \\
\hline DDO 155 & $1258 \quad 39.8$ & $+14 \quad 1310$ & 2.2 & 51 & 47.6 & 0.15 \\
\hline DDO 165 & $\begin{array}{lll}13 & 06 & 25.3\end{array}$ & $+6742 \quad 25$ & 4.6 & 89 & 61.9 & 2.26 \\
\hline DDO 167 & $\begin{array}{lll}13 & 13 & 22.9\end{array}$ & $\begin{array}{lll}+46 & 19 & 11\end{array}$ & 4.2 & -23 & 52.8 & 0.33 \\
\hline DDO 168 & $\begin{array}{lll}13 & 14 & 27.2\end{array}$ & +455546 & 4.3 & -24.5 & 54.5 & 0.83 \\
\hline DDO 187 & $\begin{array}{lll}14 & 15 & 56.7\end{array}$ & $\begin{array}{lll}+23 & 0319\end{array}$ & 2.2 & 37 & 39.0 & 0.18 \\
\hline DDO 210 & $\begin{array}{lll}20 & 46 & 52.0\end{array}$ & $-12 \quad 50 \quad 50$ & 0.9 & -85 & 66.9 & 0.17 \\
\hline DDO 216 & $\begin{array}{lll}23 & 28 & 35.0\end{array}$ & $+14 \quad 4430$ & 1.1 & -58 & 69.4 & 0.54 \\
\hline F564-V3 & $\begin{array}{lll}09 & 02 & 53.9\end{array}$ & $+2004 \quad 29$ & 8.7 & 7 & 35.8 & 0.53 \\
\hline Haro 29 & $\begin{array}{lll}12 & 26 & 16.7\end{array}$ & $+48 \quad 2938$ & 5.8 & 85 & 58.6 & 0.29 \\
\hline Haro 36 & $\begin{array}{lll}12 & 46 & 56.3\end{array}$ & +513648 & 9.3 & 2 & 37.9 & 0.68 \\
\hline IC 10 & $\begin{array}{lll}00 & 20 & 21.9\end{array}$ & $\begin{array}{l}+591739 \\
\end{array}$ & 0.7 & -38 & 41.0 & 0.40 \\
\hline IC 1613 & $\begin{array}{lll}01 & 04 & 49.2\end{array}$ & $+02 \quad 07 \quad 48$ & 0.7 & 71 & 37.9 & 0.58 \\
\hline LGS 3 & $\begin{array}{lll}01 & 03 & 55.2\end{array}$ & +215239 & 0.7 & -3.5 & 64.4 & 0.23 \\
\hline M81dwA & $\begin{array}{lll}08 & 23 & 57.2\end{array}$ & $\begin{array}{lll}+71 & 01 & 51\end{array}$ & 3.5 & 86 & 45.8 & 0.25 \\
\hline Mrk 178 & 113329.0 & +491424 & 3.9 & -50 & 68.6 & 0.33 \\
\hline NGC 1156 & $\begin{array}{lll}02 & 59 & 41.9\end{array}$ & $+25 \quad 14 \quad 21$ & 7.4 & 42 & $\ldots$ & 0.77 \\
\hline NGC 1569 & $\begin{array}{lll}04 & 30 & 49.8\end{array}$ & +645051 & 3.4 & -59 & 61.1 & 0.39 \\
\hline NGC 2366 & $\begin{array}{lll}07 & 28 & 48.8\end{array}$ & $\begin{array}{lll}+69 & 12 & 22\end{array}$ & 3.4 & 32.5 & 72.1 & 1.35 \\
\hline NGC 3738 & $\begin{array}{lll}11 & 35 & 49.0\end{array}$ & $+5431 \quad 23$ & 4.9 & 0 & 0 & 0.78 \\
\hline NGC 4163 & $\begin{array}{lll}12 & 12 & 09.2\end{array}$ & $+36 \quad 1013$ & 2.9 & 18 & 53.7 & 0.27 \\
\hline NGC 4214 & $\begin{array}{lll}12 & 15 & 39.2\end{array}$ & +361938 & 3.0 & 16 & 25.8 & 0.75 \\
\hline NGC 6822 & $1944 \quad 57.9$ & $\begin{array}{lll}-14 & 48 & 11\end{array}$ & 0.5 & 24 & $\cdots$ & 0.57 \\
\hline SagDIG & $\begin{array}{lll}19 & 30 & 00.6\end{array}$ & $\begin{array}{lll}-17 & 40 & 56\end{array}$ & 1.1 & 87.5 & 62.7 & 0.23 \\
\hline UGC 8508 & $\begin{array}{lll}13 & 30 & 44.9\end{array}$ & +545429 & 2.6 & -60 & 61.9 & 0.26 \\
\hline WLM & $\begin{array}{lll}00 & 01 & 59.2\end{array}$ & $\begin{array}{lll}-15 & 27 & 41\end{array}$ & 1.0 & -2 & 70.3 & 0.57 \\
\hline VIIZw 403 & $\begin{array}{lll}11 & 27 & 58.2\end{array}$ & +785939 & 4.4 & -10 & 66.0 & 0.52 \\
\hline
\end{tabular}

${ }^{a}$ Hunter et al. 2012

${ }^{b}$ Hunter \& Elmegreen 2004 
searching algorithm on data from Leiden-Dwingeloo and Leiden-Argentine-Bonn surveys. These automated algorithms are efficient when the parameters are identical and the structures are regular, symmetric and complete. However, such structures in galaxies have various shapes, and the data sensitivity and resolution for each sample also varies. This variation would require individual automated models for each galaxy, but this would reduce the consistency of the method. Therefore, the visual observation method gives a more consistent outcome for the discovery of holes in spite of its certain subjective biases [33].

We agree with Bagetakos et al. [33] that automated methods don't provide a satisfactory outcome when searching for such irregular structures. Therefore, we preferred visual observation over automated algorithms and searched for holes in the LITTLE THINGS sample as in Bagetakos et al. [33]. The H i structure in five galaxies of our sample were studied by Bagetakos et al. [33]. These galaxies are observed independently at first to make a 'standard' visual inspection technique. Then maintaining consistency with them, the search for H i holes was completed. We used the KARMA ${ }^{2}$ visualization software package to identify $\mathrm{H}$ I holes in the galaxies.

\subsubsection{KARMA}

The KARMA visualization software was developed for the Australia Telescope National Facility (ATNF) by Richard E. Gooch [92] to study radio images. The software performs many standard tasks like signal and image processing with the help of a structured library and API (KarmaLib) and a lot of modules. A number of interactive visualization programs like KVIS, KPVSLICE, KSHELL, KPOLAR etc. are available in KARMA. These programs use the ImageDisplay widget for a nice interface. We

\footnotetext{
${ }^{2}$ http://www.atnf.csiro.au/computing/software/karma/
} 
extensively used tasks KVIS and KPVSLICE to display our data, to characterize the holes in each region of the galaxies, and to analyze their various properties.

\section{- KVIS}

KVIS is very effective program to display and explore multiple images and three dimensional data cubes. We can view images in variety of (pseudo) color patterns and can use a histogram panel to adjust their intensity profiles. Moreover, we can overlay multiple images and their different intensity level contours, which is largely useful to compare different data-sets.

Data cubes are usually displayed channel-by-channel in increasing or decreasing order of velocity, i.e. right ascension and declination as axes for two dimensional images and velocity as the third axis. The axes can also be rotated to get better three dimensional image views of the $\mathrm{H}$ I disk.

\section{- KPVSLICE}

KPVSLICE is mostly used to display and analyze the position-velocity $(p v)$ diagrams along interactive slices through three-dimensional data cubes. Positionvelocity diagrams are two-dimensional flux density distribution maps which relate the rotational velocity and the angular position along the slices.

When we draw slices on the integrated flux density or velocity field map, we can see the $p v$ diagram simultaneously in another window. The slice can be moved and rotated freely and the corresponding position-velocity diagram will appear in the other window. The slice can be moved around interactively to compare the structures in different parts of the galaxy. The slice of the whole galaxy along its major axis gives an estimation of its rotation curve. Furthermore, KPVSLICE is very helpful to categorize the types of H I structures and to find 
Type 2 and Type 3 holes, as well as the size, position angle, expansion velocity and other parameters of the hole.

\subsubsection{Identification of Holes and Observed Properties}

In the first step, with KVIS, we searched for holes visually in the natural-weighted integrated H I maps of the entire galaxy sample and delineated them with ellipses. The coordinates of the center of the hole (in right ascension and declination, J2000) are taken as the pixel having the minimum flux density inside the ellipse closest to the visual center. For consistency, we used the same color and intensity scale throughout the sample. To approximate the flux density of those regions before the holes were created, we selected an area approximately twice the area of the hole and averaged the total flux. The estimated uncertainty of the flux is from the uncertainty of the center of the hole which is of order $10 \%$. For the quality measure of the holes, the most distinct holes with a distinct density gradient are given 3 points, down to 0 points for the most indistinct holes.

In the second step, we used the robust-weighted integrated H I maps and repeated the same process as in the first. Because of the high resolution of the data, much more structure is visible but not every structure is a hole, which makes proper identification of holes more difficult, so the highest quality measure is assigned as 2 . We did detect some holes in the robust-weighted maps which were not seen in the natural-weighted maps due to the lower resolution (as an example, holes in F564-V3). Half of the beam size (of order $3^{\prime \prime}$ ) is taken as the uncertainty of the position center of the holes in each case.

In the third step, we viewed each velocity channel map on rapid slide show (a 'movie') of the natural-weighted cube files and overlayed the ellipses drawn from the first and second steps. Only holes present in three or more consecutive channels 
were considered valid. The center of the holes were further examined with the help of channel maps to confirm that these are actual holes and not just low density regions. Another reason to view each channel map was to search for possibly 'hidden' intact holes. Those fully-contained holes can be detected well in the position-velocity diagrams and the channel maps. We quantified the quality of holes in this part as: Holes found in zero to two consecutive channels got no points. Holes seen in three to four consecutive channels got 1 point. If they are in five to seven consecutive channels they were given 2 points, and for more than seven channels we classified them as distinct holes with 3 points.

In the final step, we worked with task KPVSLICE to further quantify and analyze interactive $p v$ slices and intensity profiles through each hole in the data cubes of the sample galaxies. Intensity profiles are the flux density distribution along a slice through the center of a hole as shown in Figure 2.1. Since the slices are freely definable and the line of cut can be rotated by any angle at the center of the ellipse drawn around the holes, we can identify the major/minor axes of the ellipse, and can measure them to determine the full-width half-maximum (FWHM) of the intensity profile. The measurements are verified simultaneously in both the $p v$ diagrams and the intensity profiles. The uncertainty on the axes is of order $3^{\prime \prime}$ which is estimated as the half of the robust beam size. We also measured the position angle (PA) of the major axis from north through east. We estimated an uncertainty of order $20^{\circ}$ with multiple independent measurements. The ratio of the minor-to-major axis is the axial ratio of the hole with uncertainty of order $25 \%$ on the basis of repeated independent measurements.

The heliocentric (systemic) velocity $\left(v_{\mathrm{Hel}}\right)$ of the hole was taken as the velocity channel where the contrast between the hole and its edge is highest (i.e., where the 
clearest feature is seen) in the $p v$ diagram. The velocity resolution $\left(1.3\right.$ or $2.6 \mathrm{~km} \mathrm{~s}^{-1}$ ) is taken as an error estimation.

All the holes are classified into three groups as in Figure 2.2 using the interactive $p v$ diagram and intensity profile following Brinks \& Bajaja [49], and Bagetakos et al. [33]. Type 1 holes are completely 'blown out' holes where there is no gas visible on either the low or the high velocity side of the hole (Figure 2.1). Type 2 holes are partially blown out. They have visible gas deviating from the surrounding gas towards higher or lower velocities on one side, whereas the other side is characterized by lack of gas as shown in the $p v$ diagram. Type 3 holes are intact, where both sides can be seen in the $p v$ diagram. Examples are shown in Figure 2.2. It is expected that the expansion of Type 3 holes leads to the formation of Type 2 holes. Because Type 1 holes are generally larger than Type 2 and Type 3 [33], they are thought to be blown out and therefore are farther along the evolution process.

The expansion velocity $\left(v_{\exp }\right)$ of a hole is found by measuring the deviation of the velocity of the gas as seen in a $p v$ diagram. The expansion velocity can't be measured for completely broken (Type 1) holes. The average velocity dispersion of a nearby, quiescent region of the galaxy is taken as the upper limit of $v_{\exp }$ for these holes. For Type 2 holes, we calculated $v_{\exp }$ as the difference between the $v_{\mathrm{Hel}}$ and the gas velocity at the bump (where the gas is deviating in comparison to the surroundings). For the intact holes, we used the average difference between $v_{\mathrm{Hel}}$ and the velocities of the gas in the approaching and receding sides of the hole. The uncertainty of the calculation is again the velocity resolution $\left(1.3\right.$ or $\left.2.6 \mathrm{~km} \mathrm{~s}^{-1}\right)$.

We further assigned quality points to the holes according to the following criteria. If the intensity profile interactively displays the center of the hole as stationary across channels then the hole is given one point. A big depression in the intensity profile provides another one point. Additionally, 'sharp' edges in the $p v$ diagrams of 

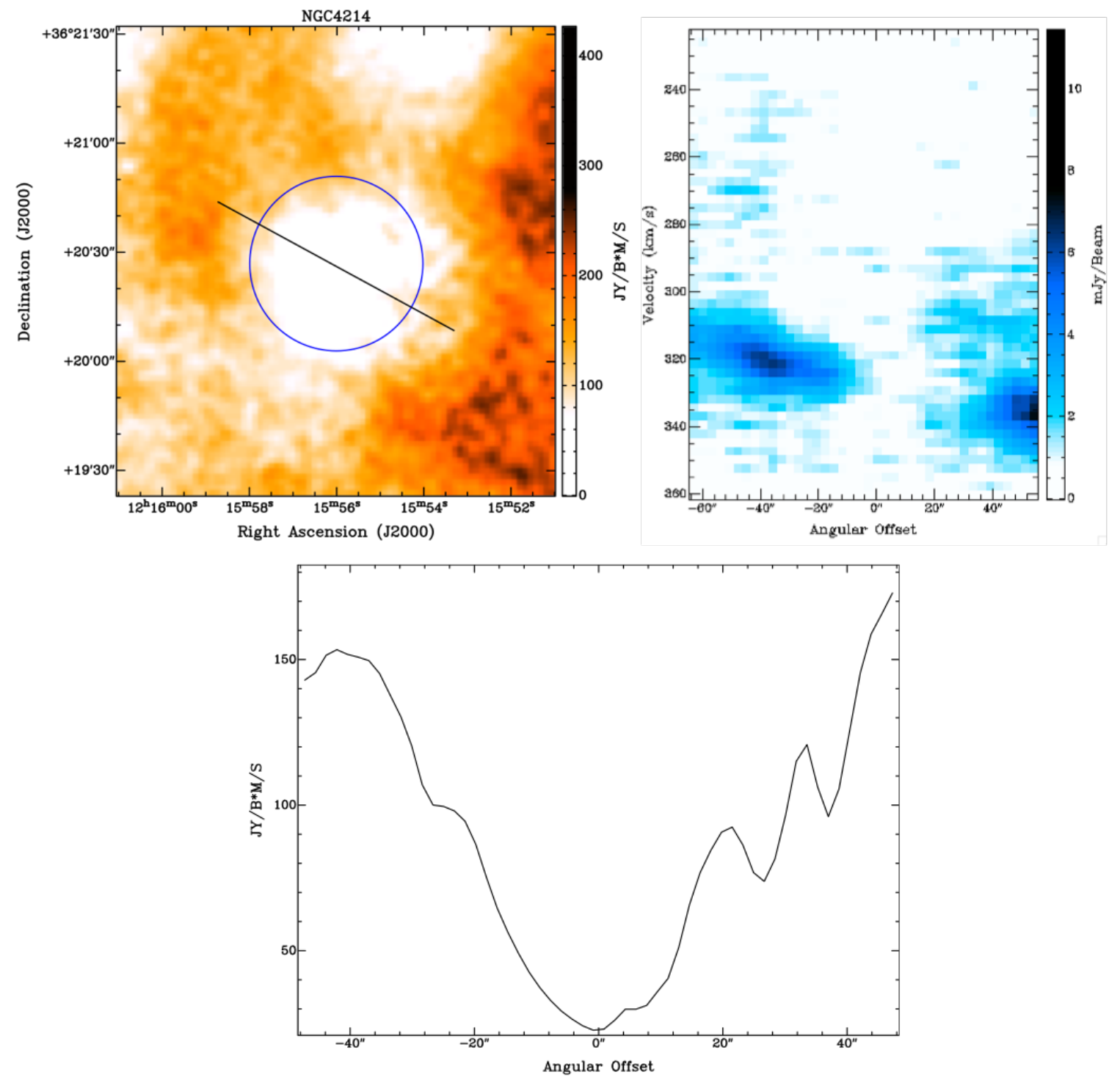

Figure 2.1: Position-velocity diagram ( $p v$ slice; upper right) and intensity profile (bottom) of a hole of NGC 4214.

Type 1 holes provide one point, a velocity deviation ('bump') in the intact side of a Type 2 hole provides one point, and if there is a bump of at least 2 channel width, it gets two points.

Finally, we added all the quality points for selection. Following the criteria of Bagetakos et al. [33], holes with a quality value less or equal to five are discarded. So, from the original list of 1181 possible holes only 306 holes are taken into account. 

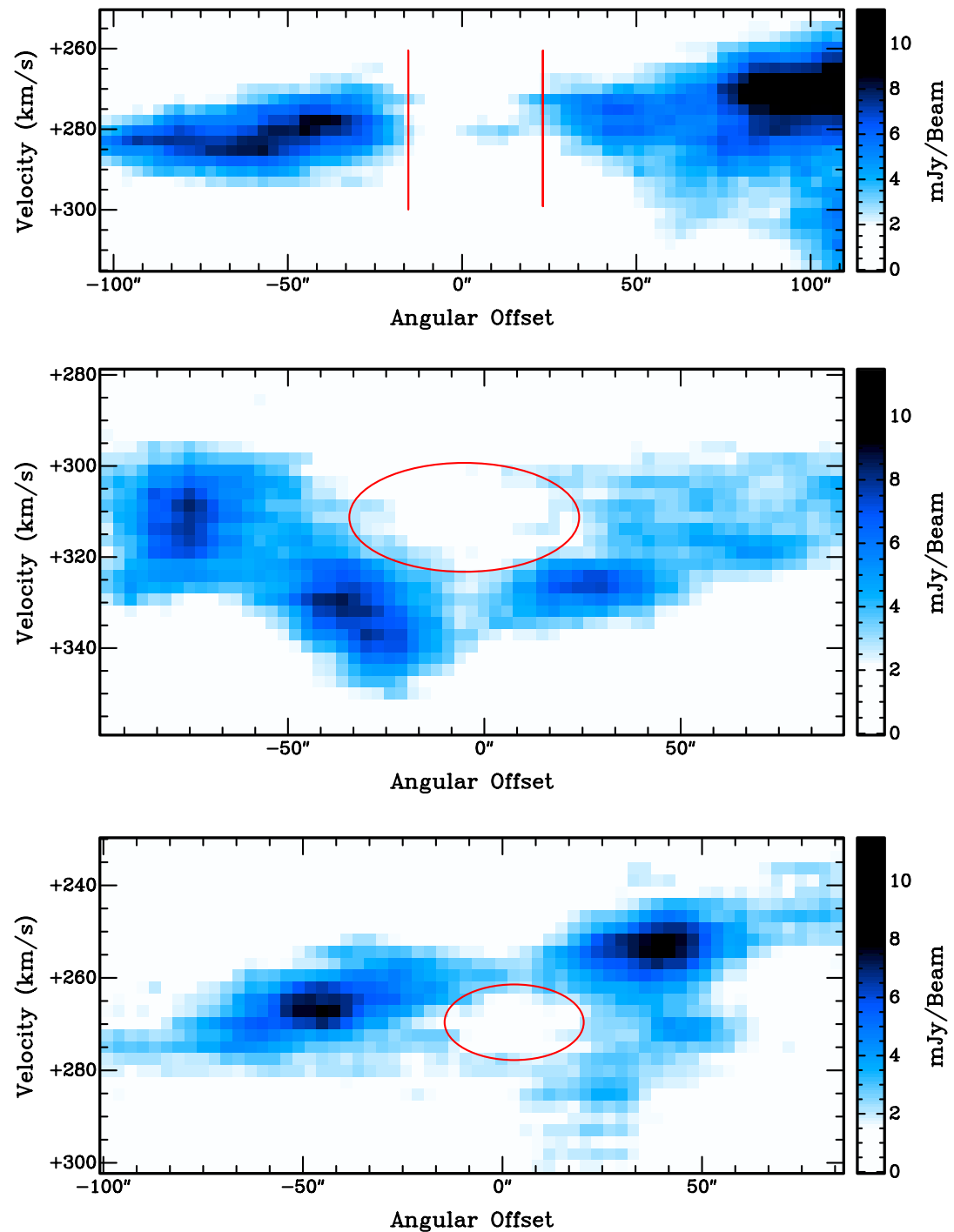

Figure 2.2: $p v$ diagrams of three types of holes (These features are from NGC 4214). Top: Type 1 hole (completely blown out); Middle: Type 2 hole (partially blown out); and Bottom: Type 3 hole (intact). 


\section{CHAPTER 3}

\section{The Catalog}

\subsection{Basic and Calculated Properties}

Table 3.1 lists the basic properties of the galaxies and holes we cataloged for each galaxy. The number of holes per galaxy in our sample ranges from 0 (NGC 4163, VII Zw 403) to 41 (DDO 50), with hole diameters (d) from 38 pc to $2.3 \mathrm{kpc}$ and expansion velocities between 5 and $30 \mathrm{~km} \mathrm{~s}^{-1}$ (recall that expansion velocities for Type 1 holes were not measurable, and so we take the average velocity dispersion in a quiet part of the galaxy as the expansion velocity limit). Nine galaxies contain only Type 1 holes (broken out-neither side visible in the $p v$ diagram) while four galaxies contain only Type 3 holes (intact-both sides visible in the $p v$ diagram). We also list the derived properties for each galaxy in Table 3.1, including the size (radius) of the H I gas disk, $R_{\max }$ defined as where the rotation curve ends or stops rising (flattens out) as seen in th $p v$ diagram taken along the galaxy's major axis. The smallest gas disk we measure is for DDO 187 with $R_{\max }=0.5 \mathrm{kpc}$; the largest is for DDO 50 with $R_{\max }=6.7 \mathrm{kpc}$. The rotation velocities, defined as the velocity at $R_{\max }$, range from $6 \mathrm{~km} \mathrm{~s}^{-1}$ for SagDIG to $77 \mathrm{~km} \mathrm{~s}^{-1}$ for NGC 4214.

The basic properties of the H I holes were used for the following fundamental calculations of the derived properties.

- The kinetic age $\left(t_{\text {kin }}\right)$ of a hole is calculated by assuming a constant expansion rate $v_{\exp }$ throughout its life time as:

$$
t_{\text {kin }}=0.978 \frac{d / 2}{v_{\exp }}
$$


Table 3.1: Basic Properties of the Holes in the LITTLE THINGS Galaxies

\begin{tabular}{|c|c|c|c|c|c|c|c|c|c|c|}
\hline Galaxies & $\begin{array}{l}\text { No. of } \\
\text { Holes }\end{array}$ & $\begin{array}{c}\text { Type } 1 \\
(\%)\end{array}$ & $\begin{array}{c}\text { Type } 2 \\
(\%)\end{array}$ & $\begin{array}{c}\text { Type } 3 \\
(\%)\end{array}$ & $\begin{array}{c}v_{\exp } \\
\left(\mathrm{km} \mathrm{s}^{-1}\right)\end{array}$ & $\begin{array}{c}d \\
(\mathrm{pc})\end{array}$ & $\begin{array}{l}R_{\max } \\
(\mathrm{kpc})\end{array}$ & $\begin{array}{c}v_{\text {rot }} \\
\left(\mathrm{km} \mathrm{s}^{-1}\right)\end{array}$ & $\begin{array}{c}v_{\text {dispersion }} \\
\left(\mathrm{km} \mathrm{s}^{-1}\right)\end{array}$ & $\begin{array}{c}Z_{0} \\
(\mathrm{pc})\end{array}$ \\
\hline CVnIdwA & 1 & 100 & 0 & 0 & 8 & 248 & 1.4 & 19 & 8 & 240 \\
\hline DDO 43 & 15 & 7 & 53 & 40 & $7-16$ & $386-797$ & 4.7 & 33 & 8 & 469 \\
\hline DDO 46 & 18 & 17 & 28 & 55 & $6-15$ & $217-743$ & 3.0 & 74 & 9 & 151 \\
\hline DDO 47 & 19 & 53 & 26 & 21 & $8-15$ & $428-1623$ & 6.6 & 40 & 9 & 608 \\
\hline DDO 50 & 41 & 61 & 20 & 19 & $8-18$ & $181-1889$ & 6.7 & 39 & 9 & 631 \\
\hline DDO 52 & 17 & 30 & 35 & 35 & $8-15$ & $382-959$ & 4.9 & 68 & 8 & 235 \\
\hline DDO 53 & 7 & 14 & 0 & 86 & $8-15$ & $126-340$ & 2.4 & 19 & 9 & 468 \\
\hline DDO 63 & 7 & 29 & 29 & 43 & $8-15$ & $208-950$ & 4.0 & $\ldots$ & 8 & $\ldots$ \\
\hline DDO 69 & 4 & 50 & 50 & 0 & $7-12$ & $38-331$ & 1.0 & 13 & 7 & 226 \\
\hline DDO 70 & 9 & 89 & 11 & 0 & $9-13$ & $171-791$ & 2.3 & 31 & 9 & 275 \\
\hline DDO 75 & 4 & 100 & 0 & 0 & 9 & $269-1195$ & 3.7 & 42 & 9 & 326 \\
\hline DDO 87 & 18 & 28 & 39 & 33 & $6-15$ & $356-1821$ & 7.1 & 45 & 7 & 456 \\
\hline DDO 101 & 2 & 0 & 50 & 50 & $10-16$ & $335-355$ & 1.8 & 72 & 9 & 92 \\
\hline DDO 126 & 4 & 75 & 0 & 25 & $9-12$ & $465-996$ & 2.9 & 36 & 9 & 294 \\
\hline DDO 133 & 5 & 100 & 0 & 0 & 10 & $494-856$ & 2.4 & 43 & 10 & 229 \\
\hline DDO 154 & 9 & 56 & 22 & 22 & $5-10$ & $183-644$ & 7.1 & 48 & 8 & 484 \\
\hline DDO 155 & 3 & 100 & 0 & 0 & 10 & $225-288$ & 0.7 & 20 & 10 & 141 \\
\hline DDO 165 & 3 & 33 & 34 & 33 & $12-13$ & $347-1797$ & 3.0 & 32 & 12 & 455 \\
\hline DDO 167 & 3 & 67 & 0 & 33 & $8-9$ & $269-391$ & 1.0 & 14 & 8 & 237 \\
\hline DDO 168 & 2 & 50 & 0 & 50 & $8-10$ & $501-751$ & 1.9 & 30 & 8 & 206 \\
\hline DDO 187 & 3 & 0 & 33 & 67 & $11-14$ & $115-173$ & 0.5 & 32 & 10 & 64 \\
\hline DDO 210 & 1 & 100 & 0 & 0 & 6 & 79 & 0.5 & 11 & 6 & 113 \\
\hline DDO 216 & 3 & 0 & 0 & 100 & $8-9$ & $147-320$ & 0.7 & 17 & 7 & 117 \\
\hline F564-V3 & 8 & 0 & 38 & 62 & $8-15$ & $588-1208$ & 2.4 & 50 & 7 & 136 \\
\hline Haro 29 & 2 & 0 & 0 & 100 & $11-13$ & $405-439$ & 2.3 & 38 & 9 & 222 \\
\hline Haro 36 & 1 & 0 & 0 & 100 & 18 & 1137 & 2.4 & 76 & 17 & 220 \\
\hline IC 10 & 20 & 70 & 25 & 5 & $13-26$ & $46-250$ & 1.5 & 52 & 16 & 189 \\
\hline IC 1613 & 11 & 100 & 0 & 0 & 6 & $143-821$ & 2.3 & 25 & 6 & 223 \\
\hline LGS 3 & 7 & 57 & 0 & 43 & $5-10$ & $27-57$ & 0.4 & 13 & 5 & 61 \\
\hline M81dwA & 10 & 10 & 10 & 80 & $7-10$ & $183-1176$ & 1.8 & 15 & 7 & 342 \\
\hline Mrk 178 & 1 & 0 & 0 & 100 & 16 & 357 & 0.9 & 14 & 10 & 253 \\
\hline NGC 1569 & 5 & 60 & 20 & 20 & $22-30$ & $228-351$ & 1.8 & 74 & 22 & 219 \\
\hline NGC 2366 & 11 & 36 & 46 & 18 & $6-15$ & $189-632$ & 6.2 & 69 & 11 & 400 \\
\hline NGC 3738 & 3 & 100 & 0 & 0 & 18 & $340-399$ & 1.4 & $\ldots$ & 18 & $\ldots$ \\
\hline NGC 4163 & 0 & 0 & 0 & 0 & $\ldots$ & $\ldots$ & 0.8 & 20 & 9 & 139 \\
\hline NGC 4214 & 21 & 71 & 19 & 10 & $9-20$ & $262-2334$ & 6.3 & 77 & 9 & 301 \\
\hline SagDIG & 1 & 100 & 0 & 0 & 9 & 666 & 1.0 & 6 & 9 & 653 \\
\hline UGC 8508 & 3 & 33 & 0 & 67 & 8 & $117-262$ & 1.4 & 32 & 8 & 144 \\
\hline WLM & 4 & 100 & 0 & 0 & 10 & $102-268$ & 0.9 & 38 & 10 & 96 \\
\hline VIIZw 403 & 0 & 0 & 0 & 0 & $\ldots$ & $\ldots$ & 1.7 & 40 & 10 & 174 \\
\hline
\end{tabular}


where $d=2 \sqrt{b_{\text {maj }} b_{\text {min }}}$ is the diameter of the hole and $b_{\text {maj }}$ and $b_{\text {min }}$ are the major and minor axes of the hole. The kinetic age, the diameter and the expansion velocity are measured in Myr, pc and $\mathrm{km} \mathrm{s}^{-1}$ respectively.

- The effective thickness $(l)$ of neutral hydrogen disk was calculated as

$$
l(r)(\mathrm{pc})=\frac{Z_{0} \sqrt{2 \pi}}{\cos i}
$$

where $i$ is the inclination of the galaxy and the scale height $\left(Z_{0}\right)$ of galaxies is given by

$$
Z_{0}(\mathrm{pc})=\frac{v_{\text {dispersion }}}{\sqrt{4 \pi G \rho(r)}}
$$

with

$$
\rho(r)=\frac{3}{4} \frac{M_{\mathrm{dyn}}}{\pi R^{3}{ }_{\max }} ; M_{\mathrm{dyn}}=\frac{v_{\mathrm{rot}}^{2} R_{\max }}{G}
$$

and the rotational velocity

$$
v_{\mathrm{rot}}=\left(\frac{\left(\left|v_{\mathrm{rot}(\max )}-v_{\mathrm{sys}}\right|+\left|v_{\mathrm{rot}(\min )}-v_{\mathrm{sys}}\right|\right) / 2}{\sin i}\right)
$$

We averaged out the velocity dispersion value in quiescent parts of the galaxy using the natural-weighted second moment maps to get an average velocity dispersion, and $M_{\text {dyn }}$ is the dynamical mass of the galaxy. The systemic velocity $\left(v_{\text {sys }}\right)$ is the velocity at the center of the sample galaxy, and is a measure of a galaxy's overall motion relative to us. The highest and the lowest values of rotational velicities, i.e., $v_{\operatorname{rot}(\max )}$ and $v_{\operatorname{rot}(\min )}$ are measured from the naturalweighted integrated $\mathrm{H}$ I intensity map and $p v$ diagram of the data cube of the 
sample galaxy. We estimated $v_{\operatorname{rot}(\max )}$ and $v_{\operatorname{rot}(\min )}$ using a $p v$ diagram along the major axis, and measured at the points where the velocity rotation curve becomes flat at its maximum and minimum values, and the distances from the center of the galaxy to those points are taken as $R_{\max }$ and $R_{\min }$ respectively.

- From 1.6, we can write the brightness temperature

$$
T_{\mathrm{B}}(\mathrm{K})=\frac{\mathrm{S}}{1.66 \times 10^{-3} \mathrm{~B}_{\mathrm{maj}} \mathrm{B}_{\text {min }}}
$$

where $B_{\text {maj }}$ and $B_{\text {min }}$, measured in arcsec, are the major and minor axes of the beam respectively. $S$ is the mean flux density in $\mathrm{mJy}_{\text {beam }}{ }^{-1}$ around the hole. From 1.7, the H I column density is given as:

$$
N_{\mathrm{H} \text { I }}\left(\mathrm{cm}^{-2}\right)=1.82 \times 10^{18} T_{\mathrm{B}}
$$

Equations 3.2, 3.6 and 3.7 are used to calculate the mid-plane H I volume density

$$
n_{\mathrm{H} \text { I }}\left(\mathrm{cm}^{-3}\right)=\frac{N_{\mathrm{H} \text { I }}}{3.08 \times 10^{18} l(r)} .
$$

- The mass of neutral hydrogen gas which is sufficient to fill up the holes was estimated as

$$
M_{\mathrm{HI}}\left(M_{\odot}\right)=0.0245 n_{\mathrm{HI}} V
$$

with the volume $(V)$ given by

$$
\begin{aligned}
& V\left(\mathrm{pc}^{3}\right)=\frac{4}{3} \pi\left(\frac{d}{2}\right)^{3} \text { for spherical holes and } \\
& V\left(\mathrm{pc}^{3}\right)=(2 \pi)^{3 / 2} Z_{0}\left(\frac{d}{2}\right)^{2} \text { for distorted holes. }
\end{aligned}
$$


- We calculated the galectocentric distance to the hole $(R)$ as

$$
R(\mathrm{pc})=D\left[\left(x^{\prime \prime}\right)^{2}+\left(y^{\prime \prime}\right)^{2}\right]^{\frac{1}{2}}
$$

where $D(\mathrm{pc})$ is the distance of the galaxies from the Sun, and $x^{\prime \prime}$ and $y^{\prime \prime}$ are given by

$$
\begin{aligned}
x^{\prime \prime} & =x \sin \theta+y \cos \theta, \\
y^{\prime \prime} & =\frac{x \cos \theta-x \cos \theta}{\cos i}, \\
x & =\left(\alpha-\alpha_{0}\right) \cos \delta_{0}, \text { and } \\
y & =\delta-\delta_{0} .
\end{aligned}
$$

Here, $(\alpha, \delta)$ and $\left(\alpha_{0}, \delta_{0}\right)$ are the coordinates of the center of the hole and the center of the galaxy respectively, and $\theta$ is the position angle of the galaxy's major axis.

- The estimation of the energy required to form a hole by stellar winds and/or SNe is calculated by two different methods. In the first method, we followed Chevalier (1974) [93]. This gives an estimation of the energy from a single supernova explosion and uses the current expansion velocity $\left(v_{\exp }\right)$, the diameter of the hole $(d)$ and the volume density of gas $\left(n_{0}\right)$. Since the amounts of other components like $\mathrm{He}$ and $\mathrm{H}_{2}$ are negligible, $n_{0}$ is replaced by the volume density $\left(n_{\mathrm{HI}}\right)$ of neutral hydrogen.

$$
E_{\mathrm{Ch}}(\mathrm{erg})=5.3 \times 10^{43} n_{0}^{1.12}\left(\mathrm{~cm}^{-3}\right)\left(\frac{d(\mathrm{pc})}{2}\right)^{3.12} v_{\exp }^{1.4}\left(\mathrm{~km} \mathrm{~s}^{-1}\right)
$$


The second estimation is based on McCray and Kafatos (1977) [57] which considers multiple explosions of supernovae for the creation of a hole. It is given as:

$$
E_{\mathrm{Mc}}(\mathrm{erg})=n_{0}\left(\frac{d(\mathrm{pc})}{194}\right)^{2}\left(\frac{v_{\mathrm{exp}}}{5.7}\right)^{3} \times 10^{51}
$$

\subsection{LITTLE THINGS Galaxies and Their Properties}

A description of all the LITTLE THINGS galaxies, H I structures and their properties are presented in the following sections. 


\subsubsection{CVnIdwA}

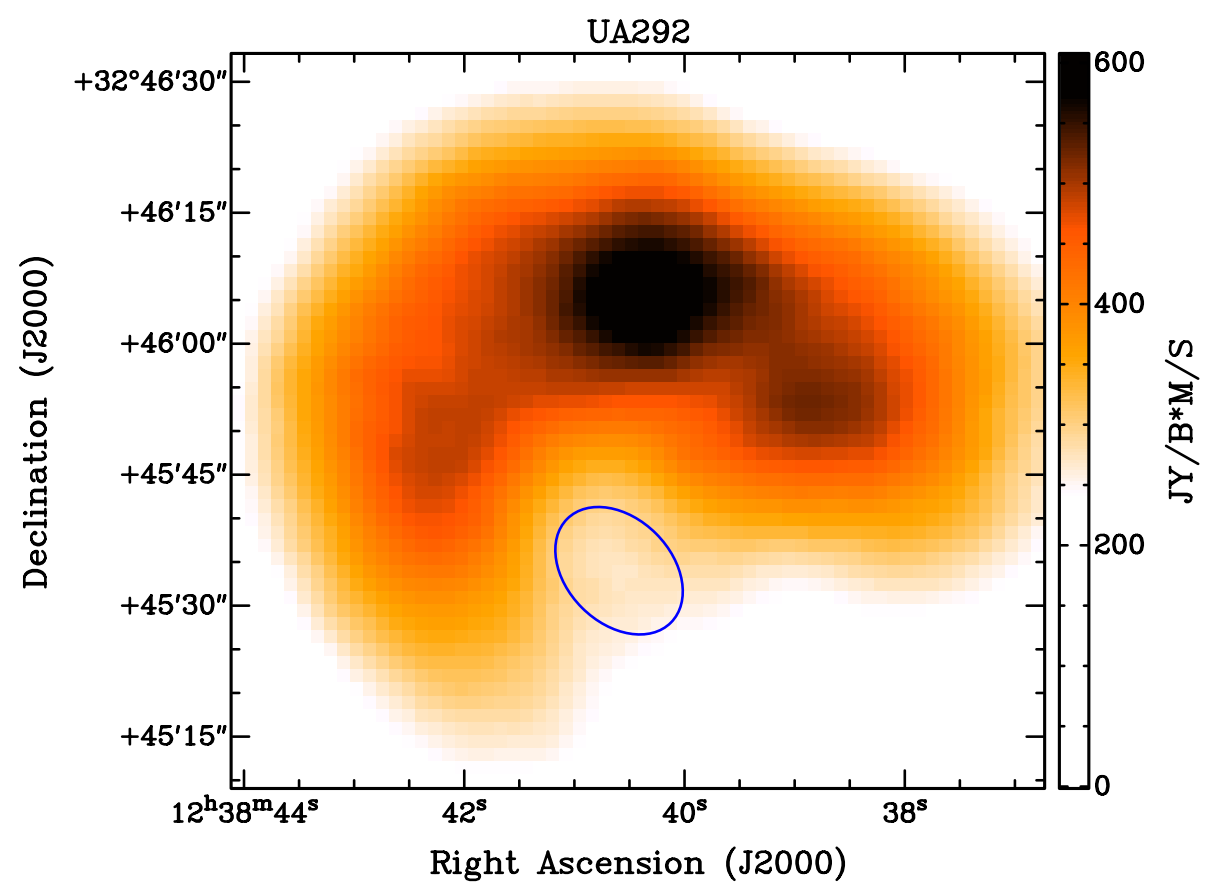

Figure 3.1: H I hole in the ISM of CVnIdwA (marked with ellipse). Blue ellipse indicates that it is Type 1 hole.

Canes Venatici I dwarf A (CVnIdwA) or UGCA 292 is an extremely metal-poor dwarf galaxy [94] in the Canes Venatici constellation. The galaxy is situated about 3.6 Mpc away from the Milky Way with V-band magnitude -12.4, and has only one hole: a Type 1 hole of diameter $\approx 288$ pc. The estimated H I disk length and the scale height of the galaxy are about $1.4 \mathrm{kpc}$ and $240 \mathrm{pc}$ respectively. 


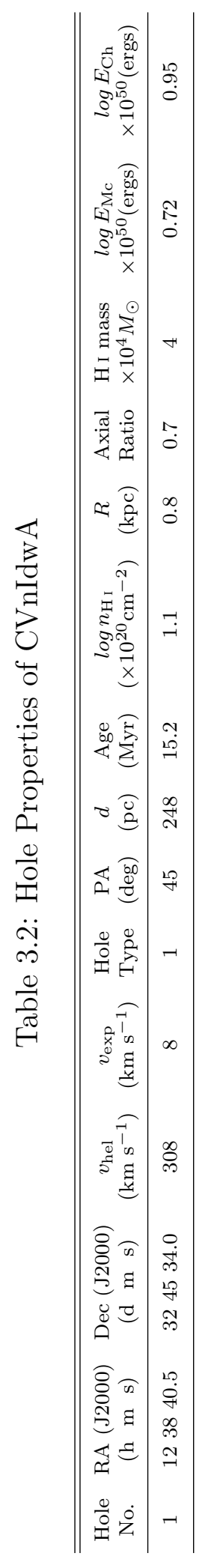




\subsubsection{DDO 43}

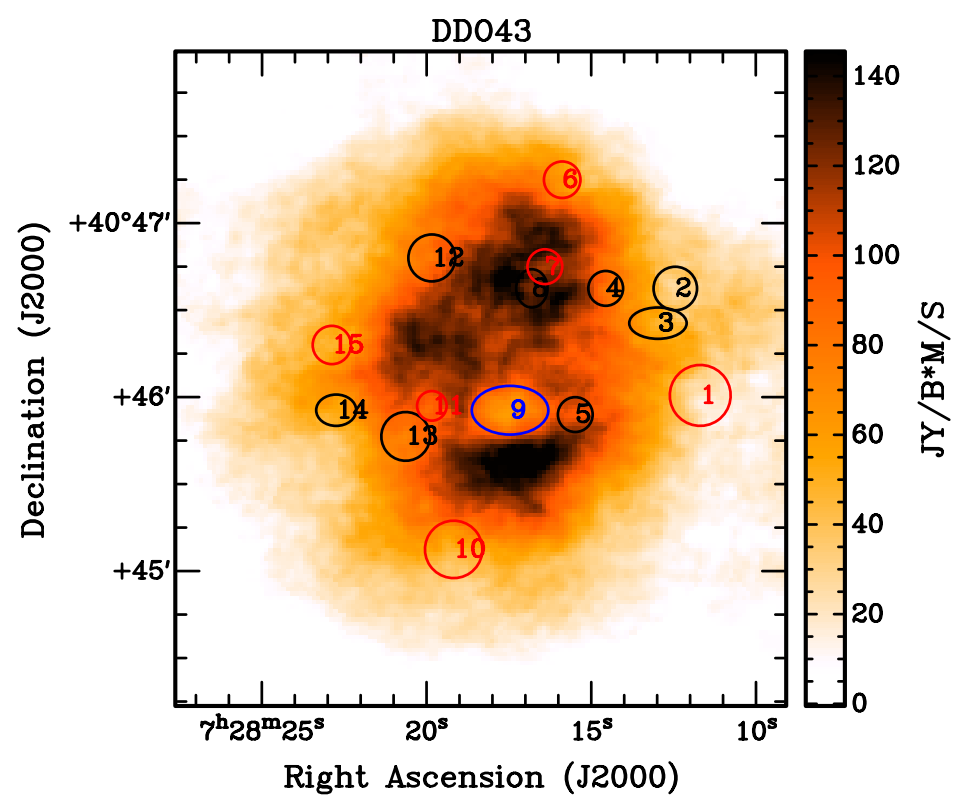

Figure 3.2: H I holes in the ISM of DDO 43 (marked with ellipses). Numbers inside the ellipse indicate the hole number from the catalog. Colors show the types of the holes: Blue ellipses are for Type 1, black are for Type 2, and red are for Type 3 holes.

DDO 43 (PGC 21073, UGC 3860) is located 7.8 Mpc away in the Ursa Major constellation. Although it is a member of the NGC 2841 Group, it is relatively isolated from the other members. Our estimation of the scale height is about 469 pc. Simpson et al. in 2005 [95] identified four apparent H I holes in the ISM of this galaxy. We detected 15 holes with most of them being Type 2 and Type 3 . We found only one Type 1 hole which is the largest hole (also the largest hole in Simpson et al. [95]) in the galaxy. 


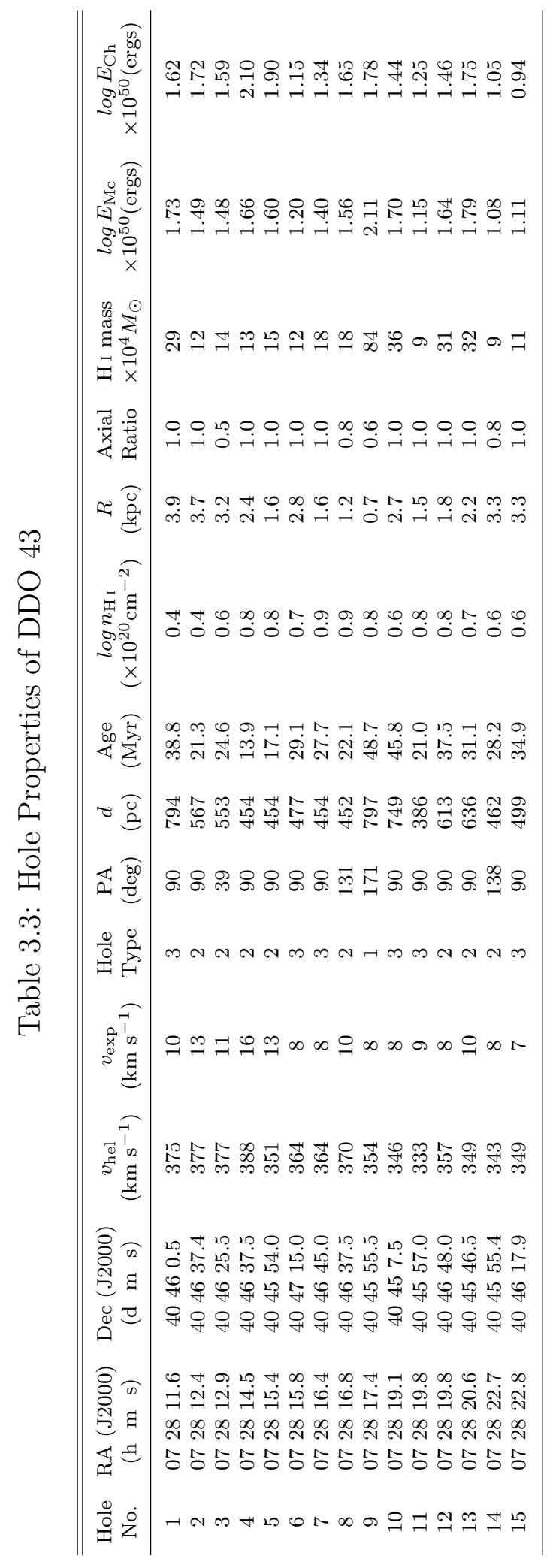




\subsubsection{DDO 46}

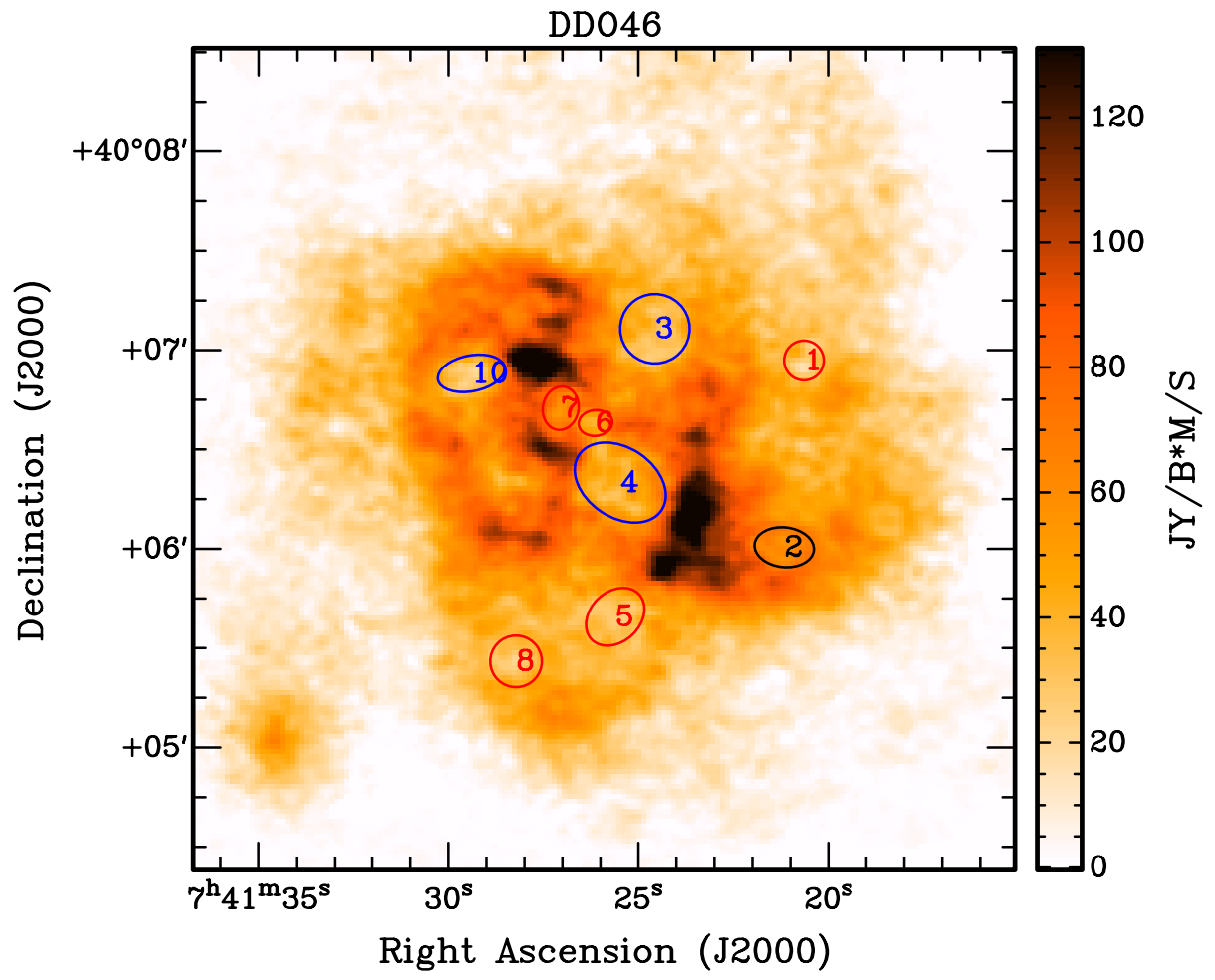

Figure 3.3: H I holes in the ISM of DDO 46 (marked with ellipses). Numbers inside the ellipse indicate the hole number from the catalog. Colors show the types of the holes: Blue ellipses are for Type 1, black are for Type 2, and red are for Type 3 holes.

DDO 46 (PGC 21585, UGC 3966) is situated 6.1 Mpc away with V-band magnitude -14.7. The galaxy has $18 \mathrm{H}$ i holes and more than $50 \%$ of those holes are Type 3 . The estimated scale height and H I disk length are about 151 pc and 3 kpc respectively. 


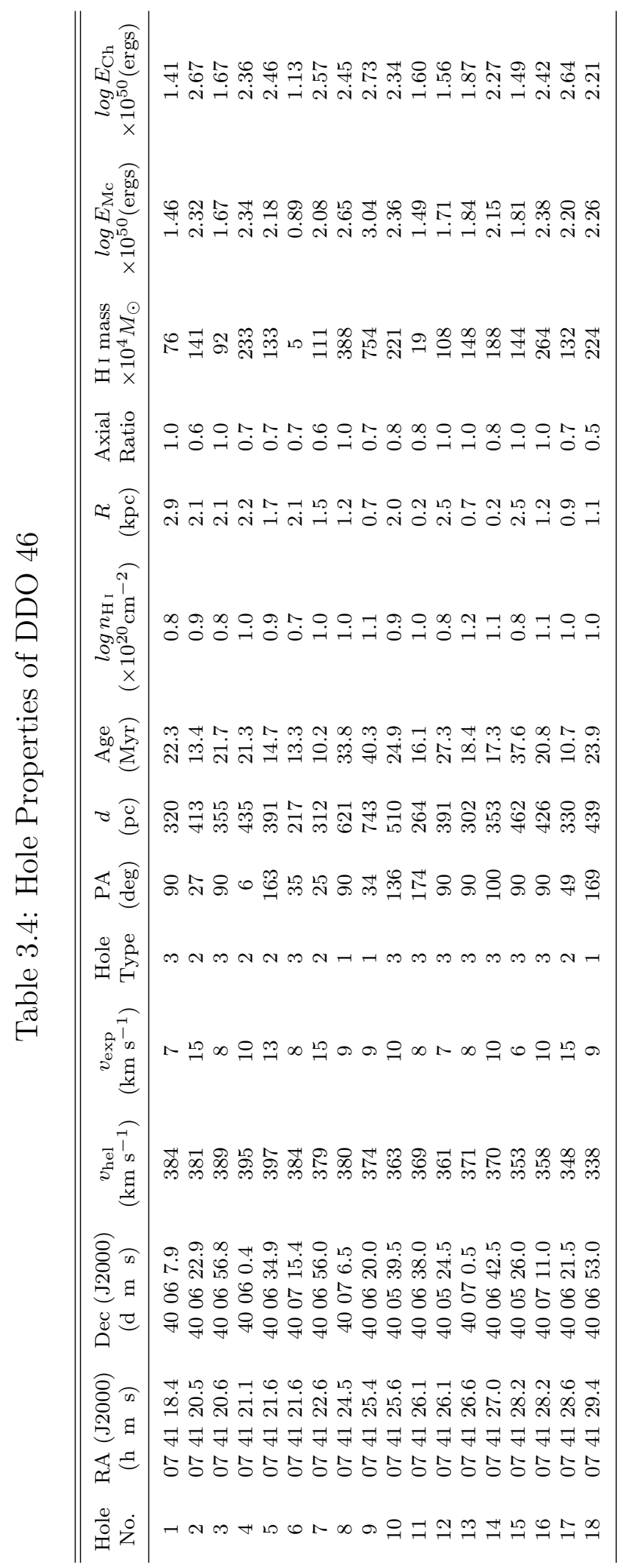




\subsubsection{DDO 47}

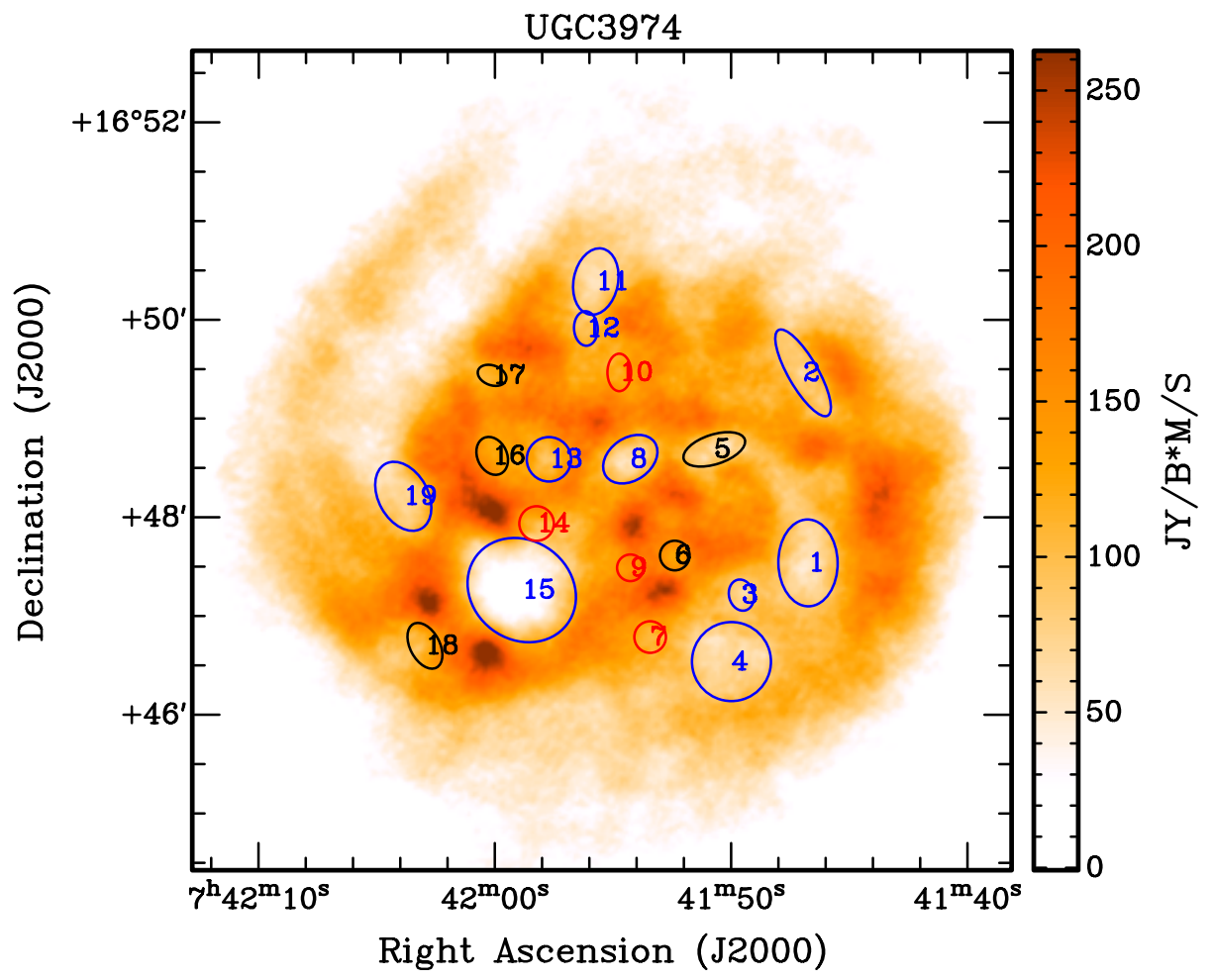

Figure 3.4: H I holes in the ISM of DDO 47 (marked with ellipses). Numbers inside the ellipse indicate the hole number from the catalog. Colors show the types of the holes: Blue ellipses are for Type 1, black are for Type 2, and red are for Type 3 holes.

DDO 47 (PGC 21600, UGC 3974) is an isolated gas-rich dwarf about 5.2 Mpc away. It has 19 holes, about half of which are Type 1, including a supershell (No. 15), which were found in the previous study done by Walter and Brinks [96]. However, we detected another large hole (No. 4) which is not in their list. Our estimation of the scale height of DDO 47 is 608 pc. 


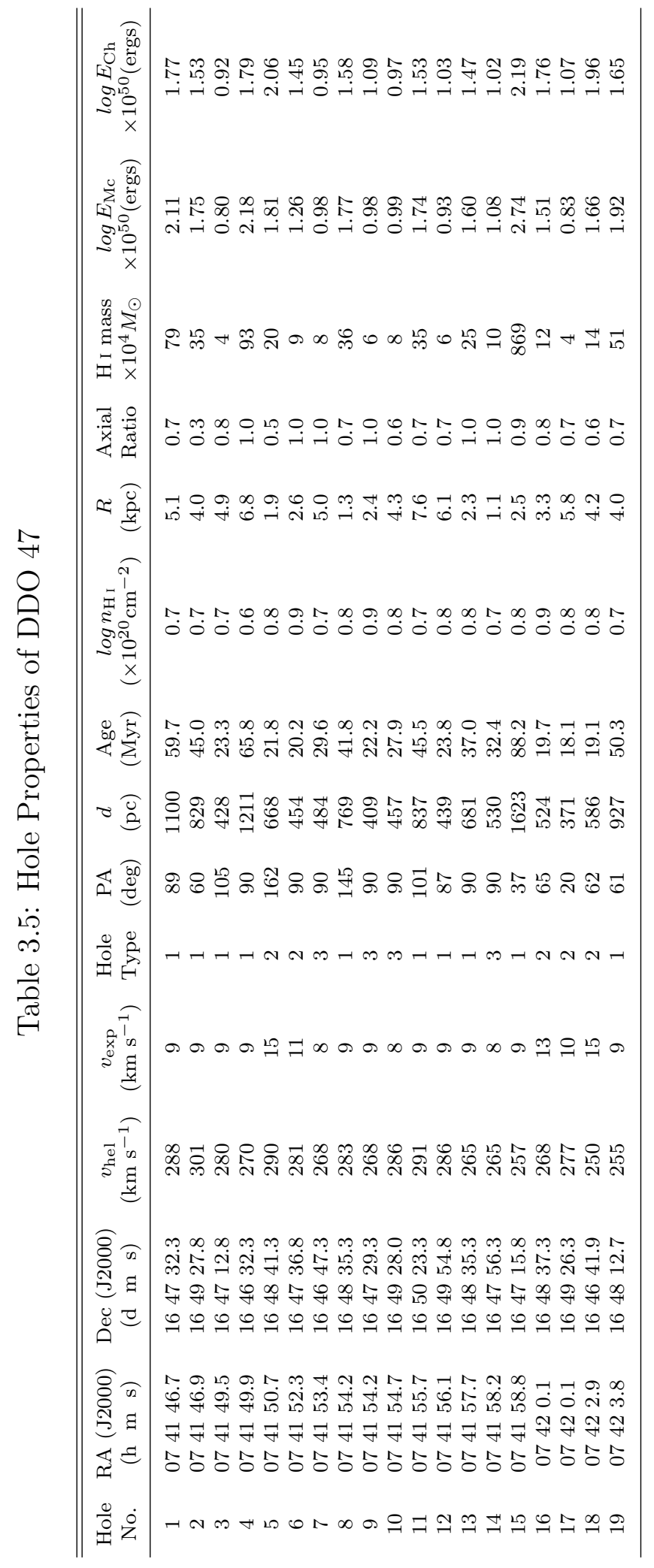




\subsubsection{DDO 50}

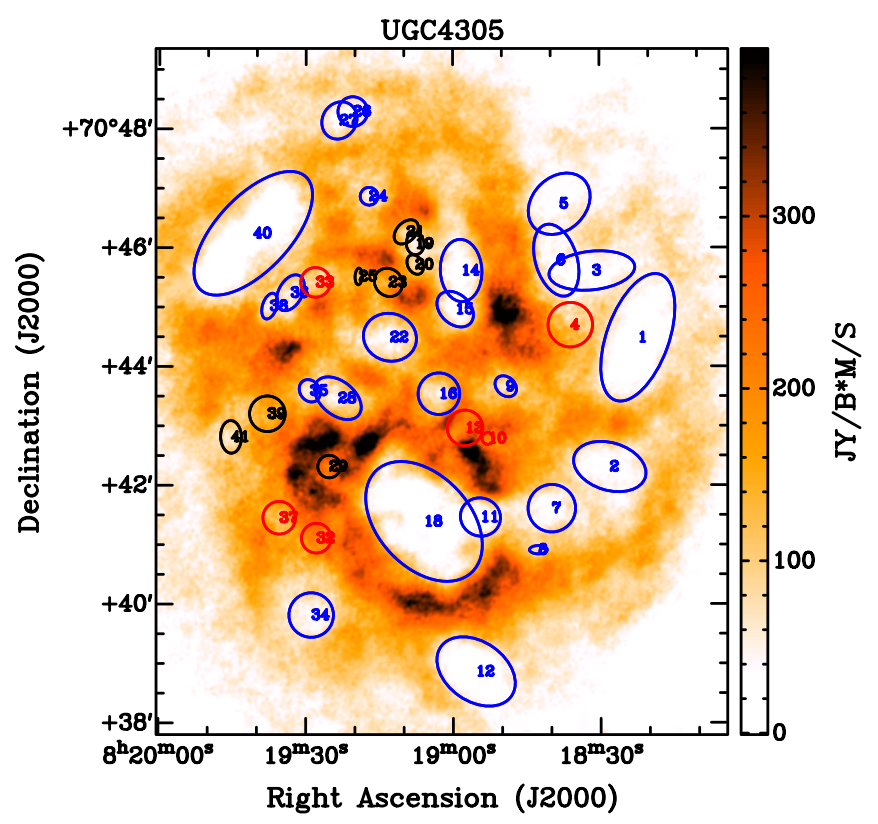

Figure 3.5: H I holes in the ISM of DDO 50 (marked with ellipses). Numbers inside the ellipse indicate the hole number from the catalog. Colors show the types of the holes: Blue ellipses are for Type 1, black are for Type 2, and red are for Type 3 holes.

DDO 50 or Holmberg II, also known as UGC 4305, PGC 23324 and VIIZw 223, is one of the largest dwarf galaxies in the LITTLE THINGS sample. It is an M81 Group galaxy with magnitude -16.6 , located at a distance of about $3.4 \mathrm{Mpc}$. Previous studies of $\mathrm{H}$ I holes in DDO 50 done by Puche et al. [50] detected 51 holes, whereas Bagetakos et al. [33] restricted the number of holes to 39. The location and size of most of those holes are comparable in both papers. Our hole search resulted in 41 holes in DDO 50. For consistency, we compared the properties of each structure with Bagetakos et al. [33] and we found that our result is in nice agreement, but we still have fewer holes than Puche et al. [50] probably because of our selection criteria. Some of the holes in Puche et al. [50] are considered as a large single hole in our list. 


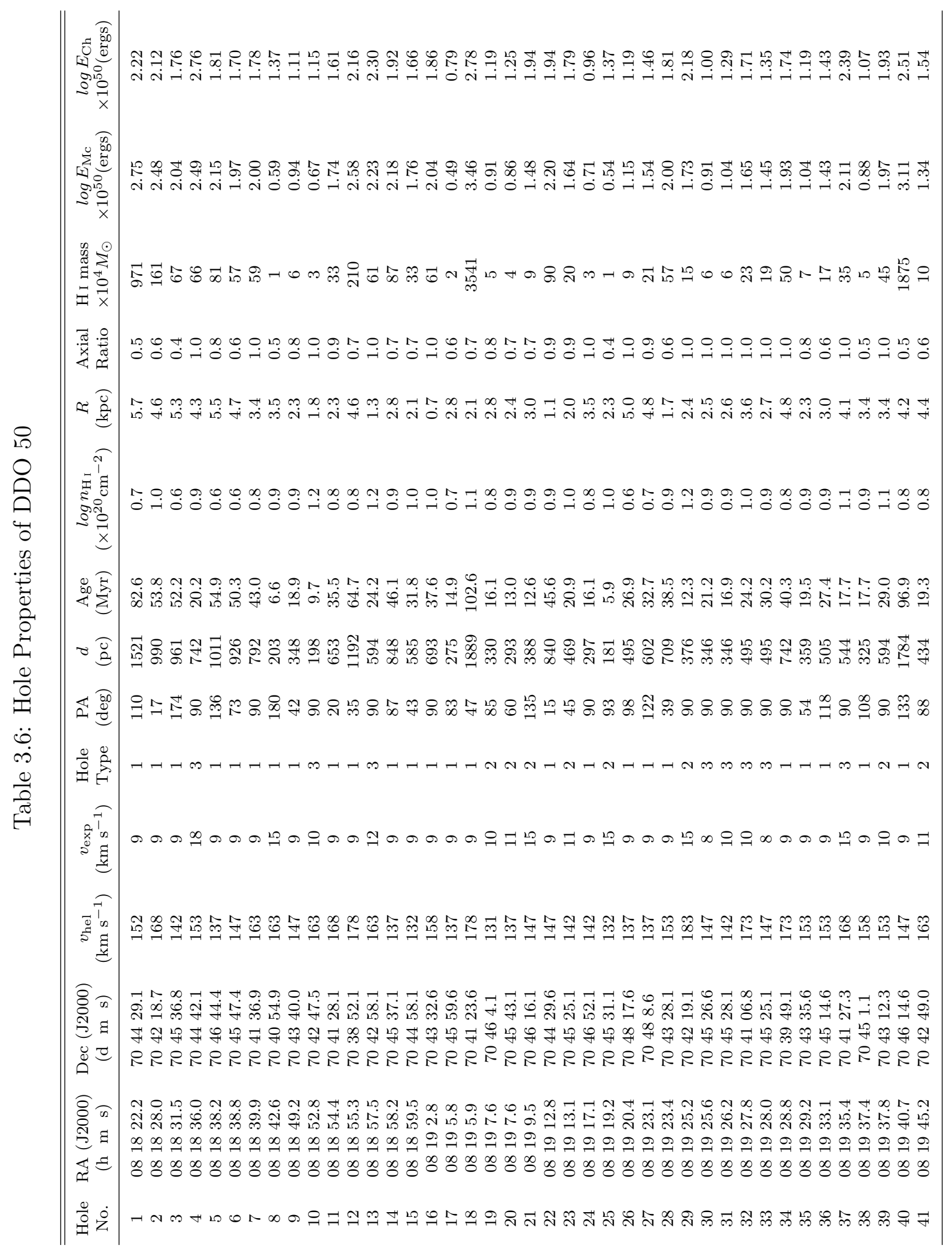




\subsubsection{DDO 52}

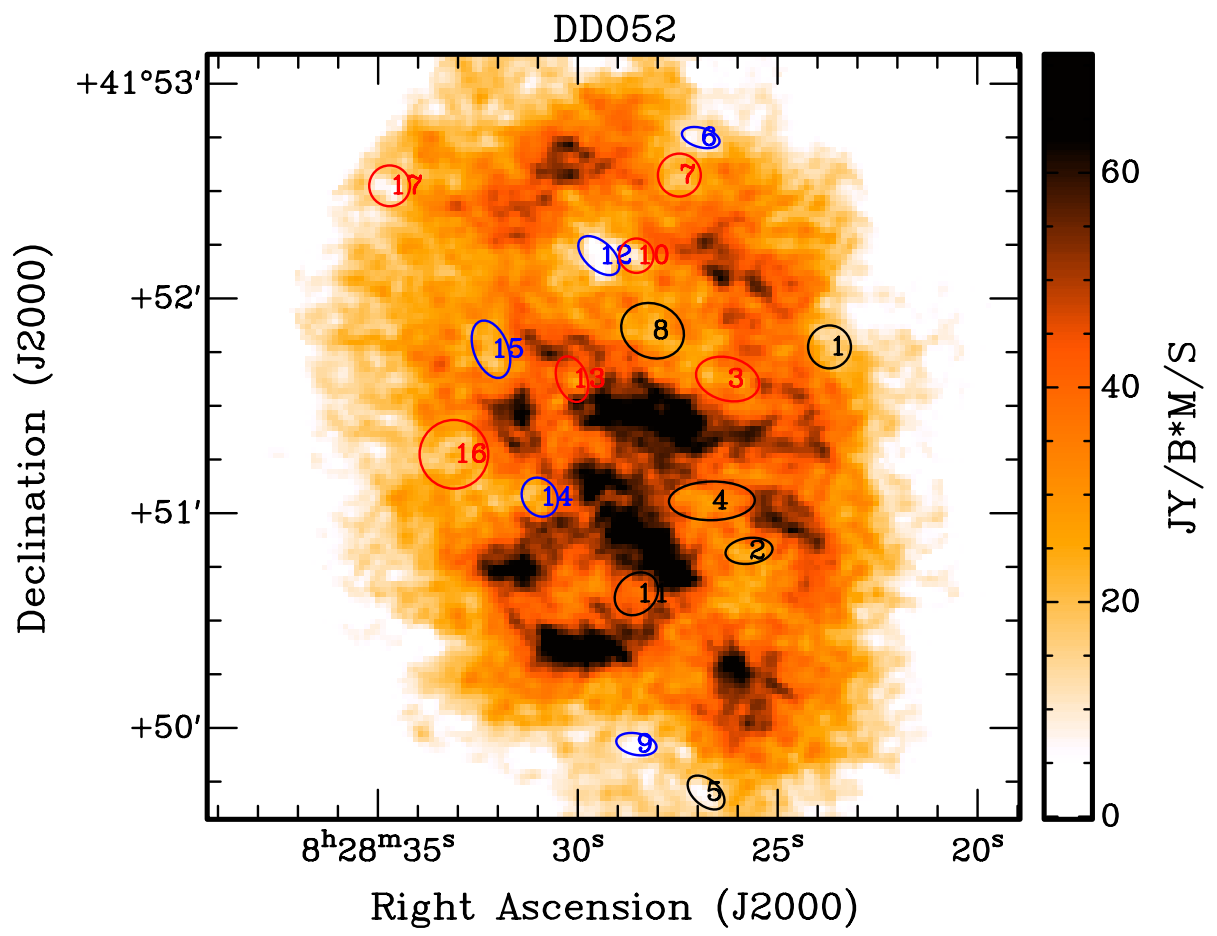

Figure 3.6: H I holes in the ISM of DDO 52 (marked with ellipses). Numbers inside the ellipse indicate the hole number from the catalog. Colors show the types of the holes: Blue ellipses are for Type 1, black are for Type 2, and red are for Type 3 holes.

DDO 52 (PGC 23769, UGC 4426), which lies about $10.3 \mathrm{Mpc}$ away, is the most distant galaxy in the LITTLE THINGS sample. It is an isolated galaxy in the NGC 2841 Group $\left(\mathrm{NED}^{1}\right)$. The calculated scale height of the galaxy is about $235 \mathrm{pc}$, and the $\mathrm{H}$ I disk extends to $\approx 4.9 \mathrm{kpc}$ from the galactic center. We detected $17 \mathrm{H} \mathrm{I}$ holes in the disk according to our selection criteria.

\footnotetext{
${ }^{1}$ The NASA/IPAC Extragalactic Database (NED) is operated by the Jet Propulsion Laboratory, California Institute of Technology, under contract with the National Aeronautics and Space Administration.
} 


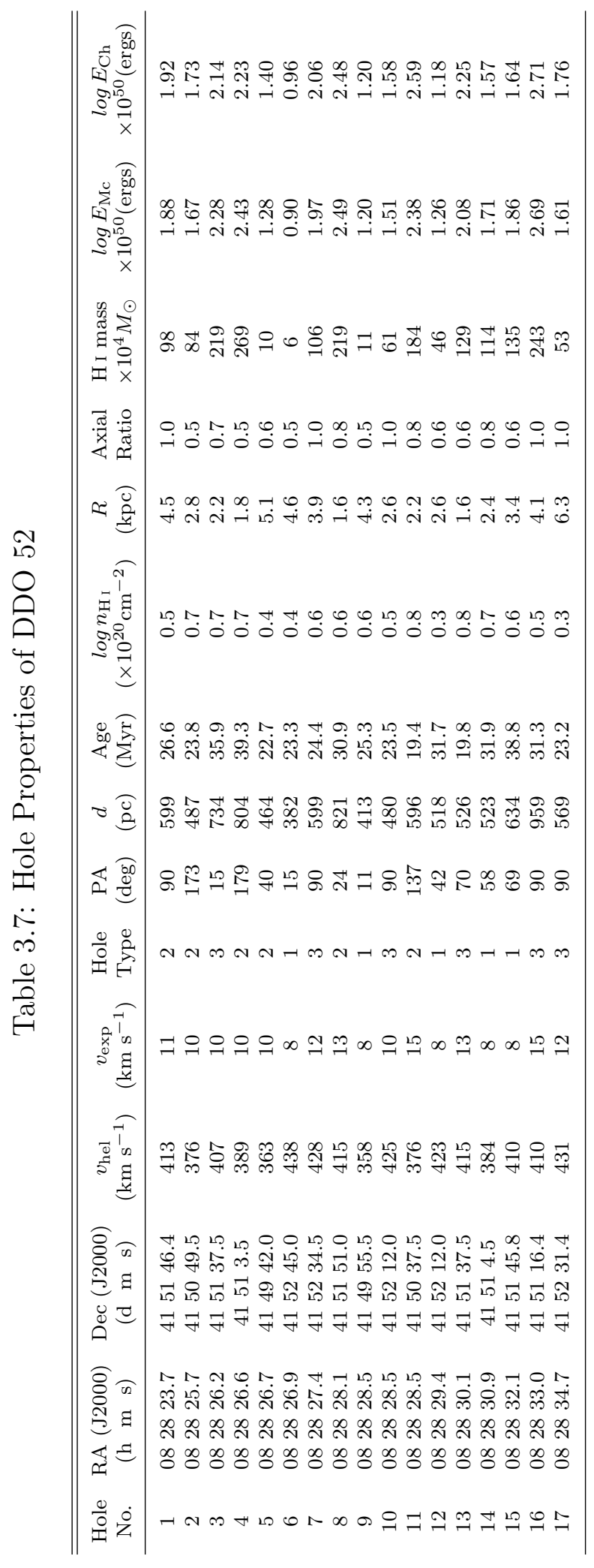




\subsubsection{DDO 53}

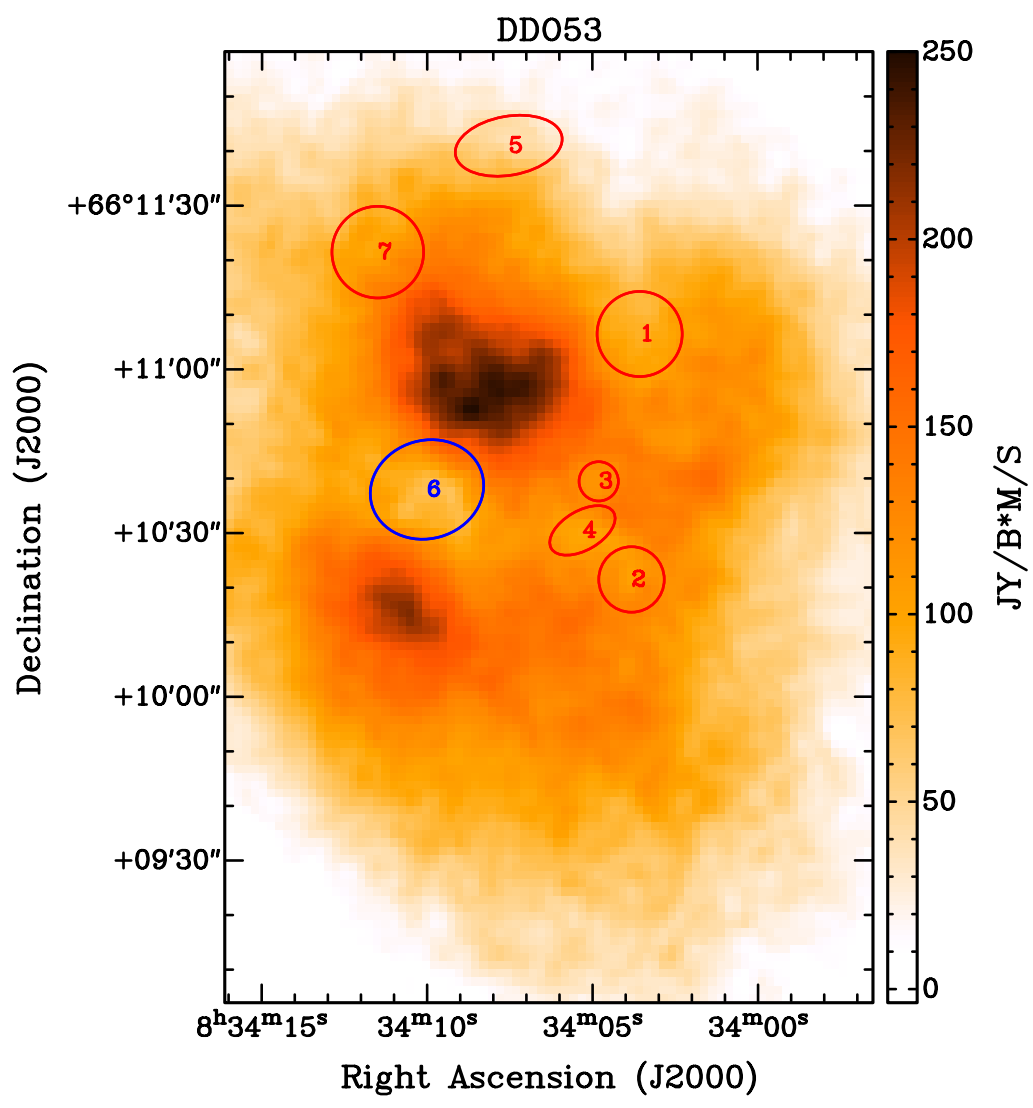

Figure 3.7: H I holes in the ISM of DDO 53 (marked with ellipses). Numbers inside the ellipse indicate the hole number from the catalog. Colors show the types of the holes: Blue ellipses are for Type 1, and red are for Type 3 holes.

DDO 53 (PGC 24050, UGC 4459, VIIZw 238) is another M81 Group member located 3.6 Mpc away with magnitude -13.8 . We found a Type 1 hole and six Type 3 holes in the galaxy which is more than double the number detected by Bagetakos et al. [33]. The estimation of the scale height of the galaxy is $468 \mathrm{pc}$. 


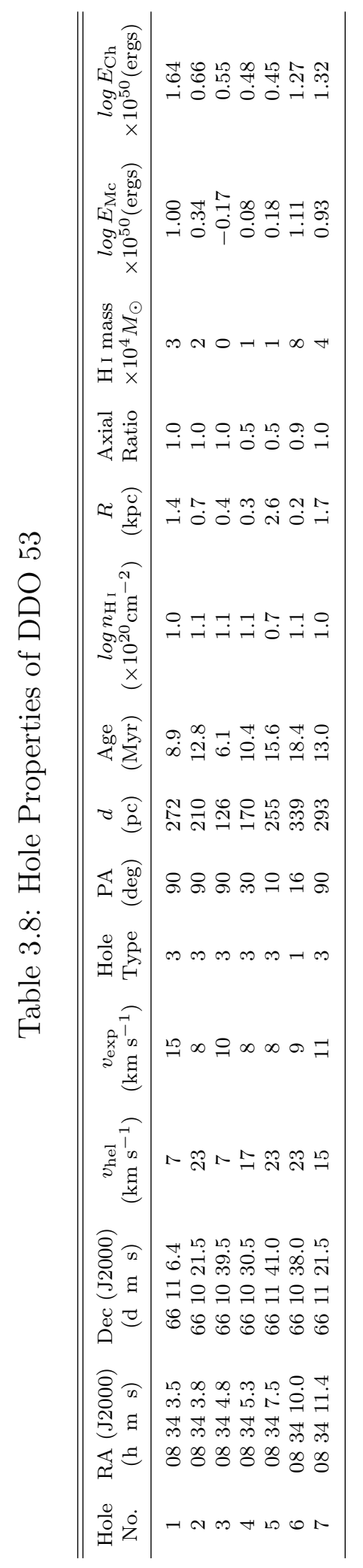




\subsubsection{DDO 63}

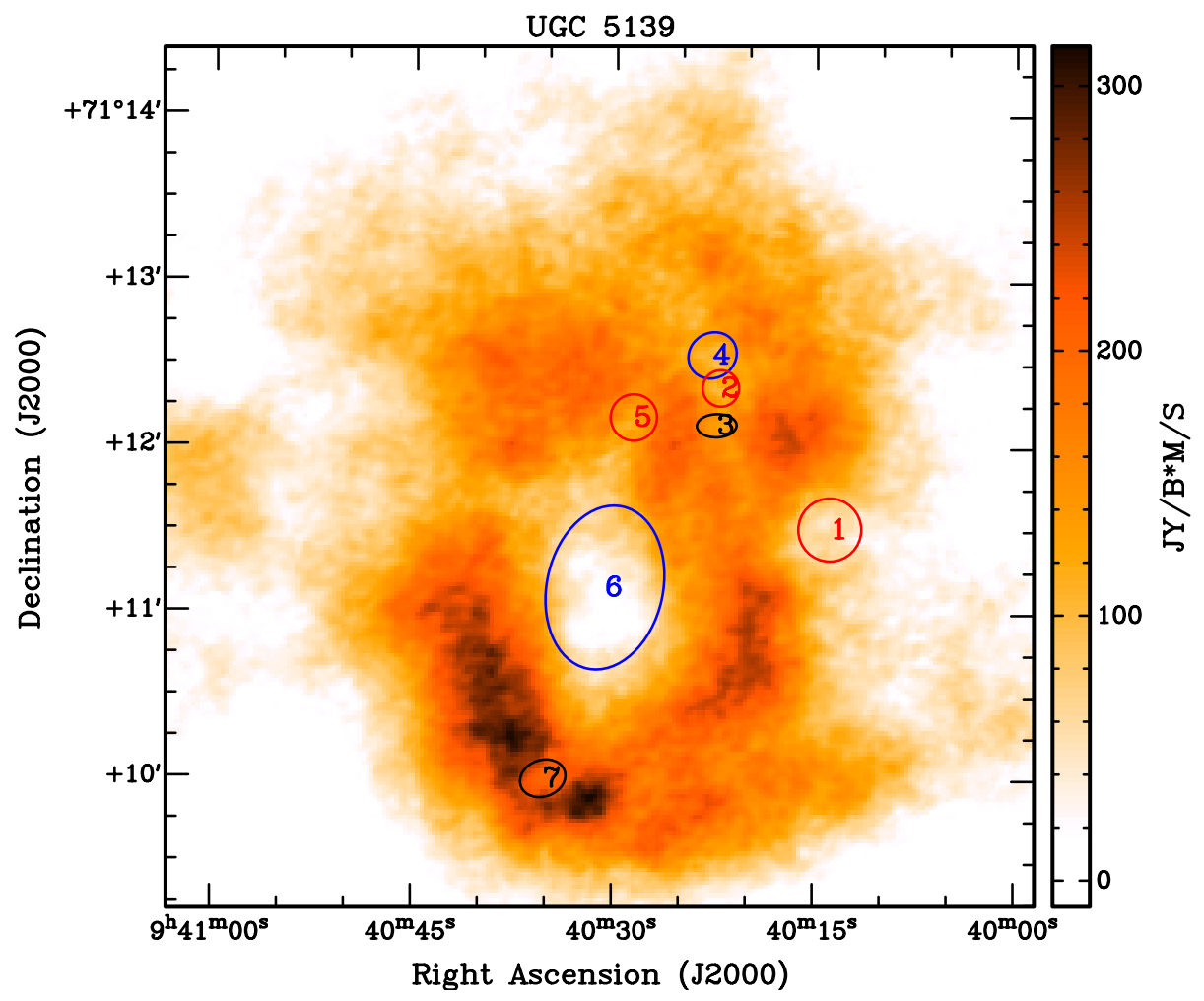

Figure 3.8: H I holes in the ISM of DDO 63 (marked with ellipses). Numbers inside the ellipse indicate the hole number from the catalog. Colors show the types of the holes: Blue ellipses are for Type 1, black are for Type 2, and red are for Type 3 holes.

DDO 63 (Holmberg I, UGC 5139, PGC 27605) is also a member of the M81 Group, located at a distance of $3.9 \mathrm{Mpc}$, and dominated by a large Type 1 hole. The first detailed study of the H I structure was done by Ott et al. [59] who discovered one large hole. Later, Bagetakos et al. [33] found six holes, and our analysis resulted in finding seven holes in the galaxy including the large hole first discovered by Ott et al. [59]. Our estimation of the properties of the holes are in good agreement with both previous studies. The scale height and other properties of, of the galaxy couldn't be estimated because of its face-on $\left(i=0^{\circ}\right)$ inclination [84]. 


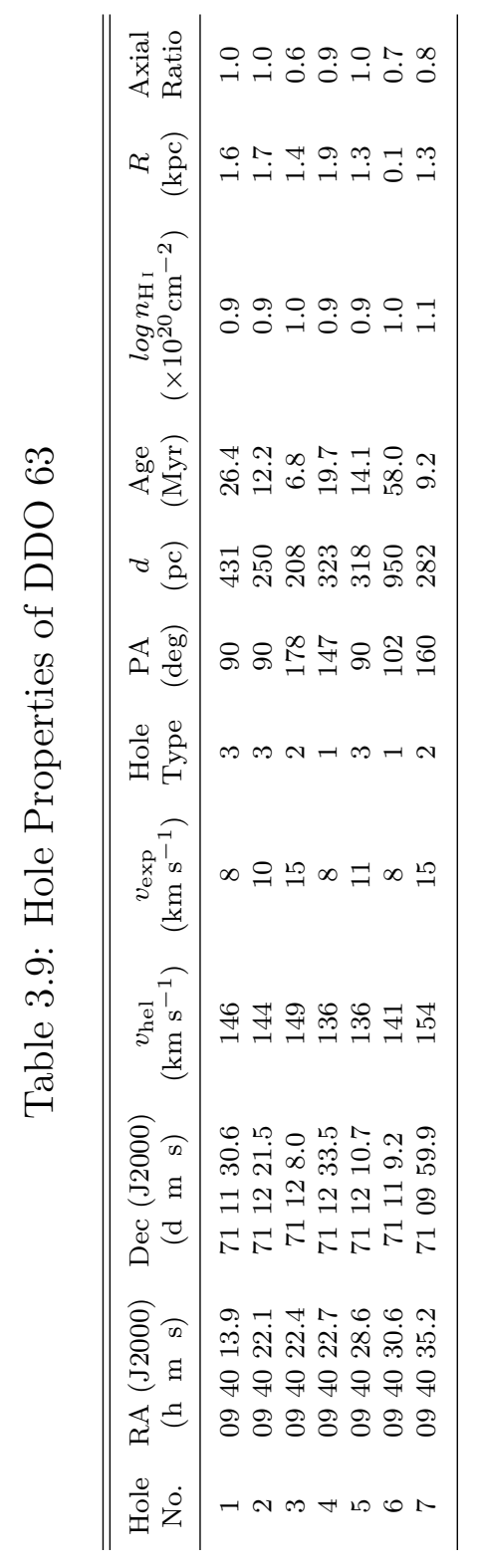




\subsubsection{DDO 69}

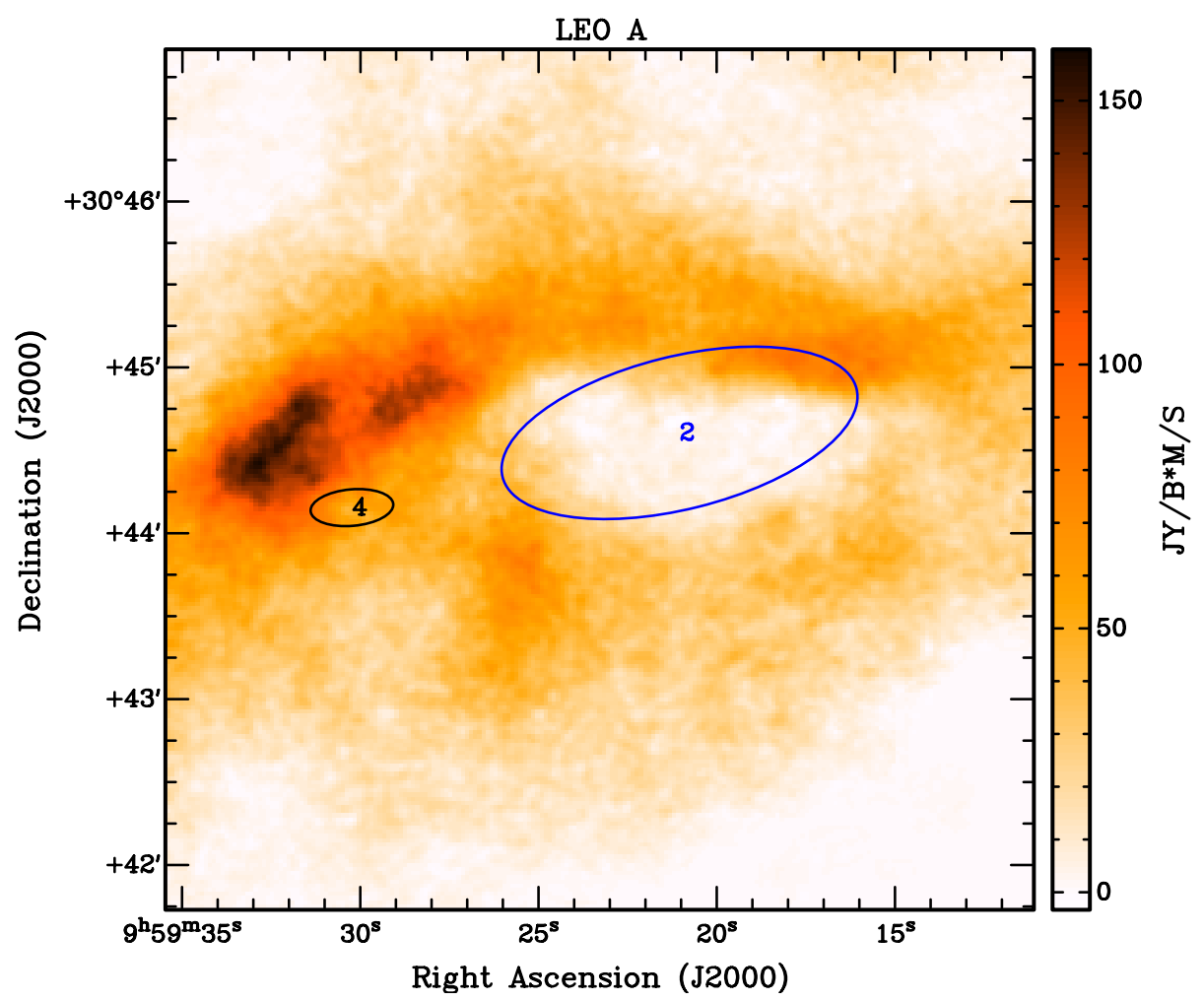

Figure 3.9: H I holes in the ISM of DDO 69 (marked with ellipses). Numbers inside the ellipse indicate the hole number from the catalog. Colors show the types of the holes: Blue ellipse is for Type 1, and black is for Type 2 hole.

DDO 69 (PGC 28868, UGC 5364, Leo A) is a Local Group galaxy and Milky Way satellite. It is $0.8 \mathrm{Mpc}$ away with $\mathrm{V}$-band magnitude -11.7. The galactic disk is dominated by a Type 1 hole (No.4), and the rest of the holes are quite small in size. The H I disk of the galaxy extends to $\approx 1 \mathrm{kpc}$. 


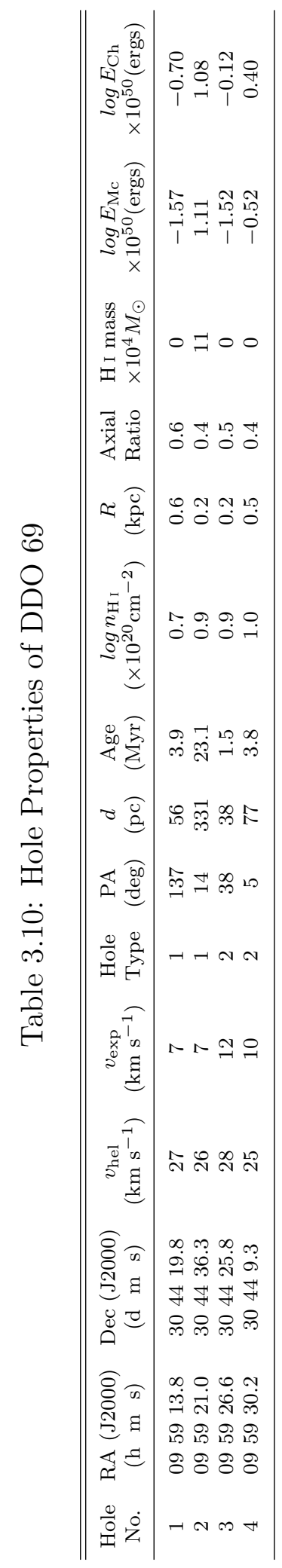




\subsubsection{DDO 70}

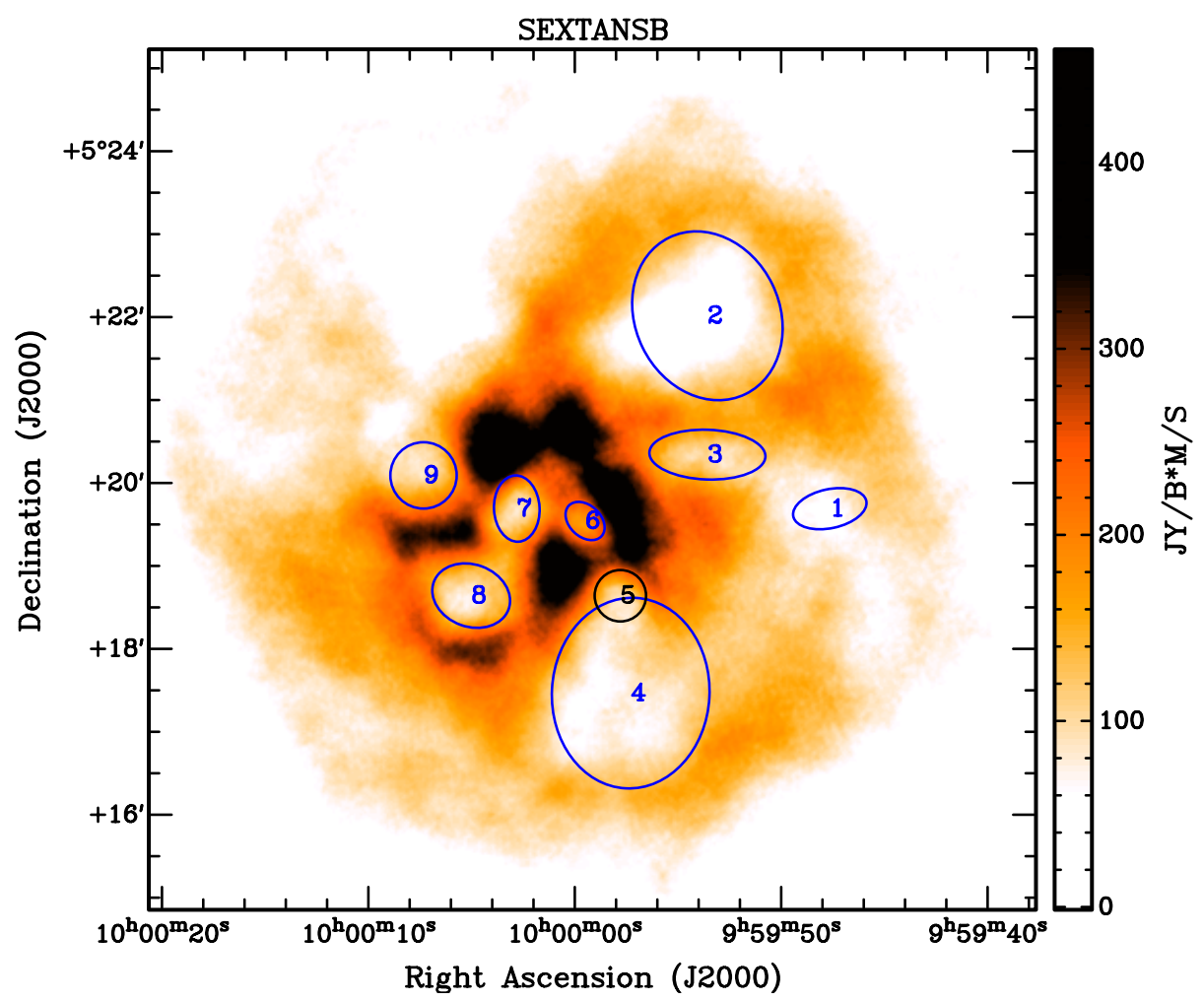

Figure 3.10: H I holes in the ISM of DDO 70 (marked with ellipses). Numbers inside the ellipse indicate the hole number from the catalog. Colors show the types of the holes: Blue ellipses are for Type 1, and black are for Type 2 holes.

DDO 70 (UGC 5373, PGC 28913), most commonly known as Sextans B, is one of the most distant members of the Local Group of galaxies. It is situated $\approx 1.3 \mathrm{Mpc}$ away from the Milky Way in the Sextans constellation. The galactic disk has nine H I holes, and eight of them are Type 1. 


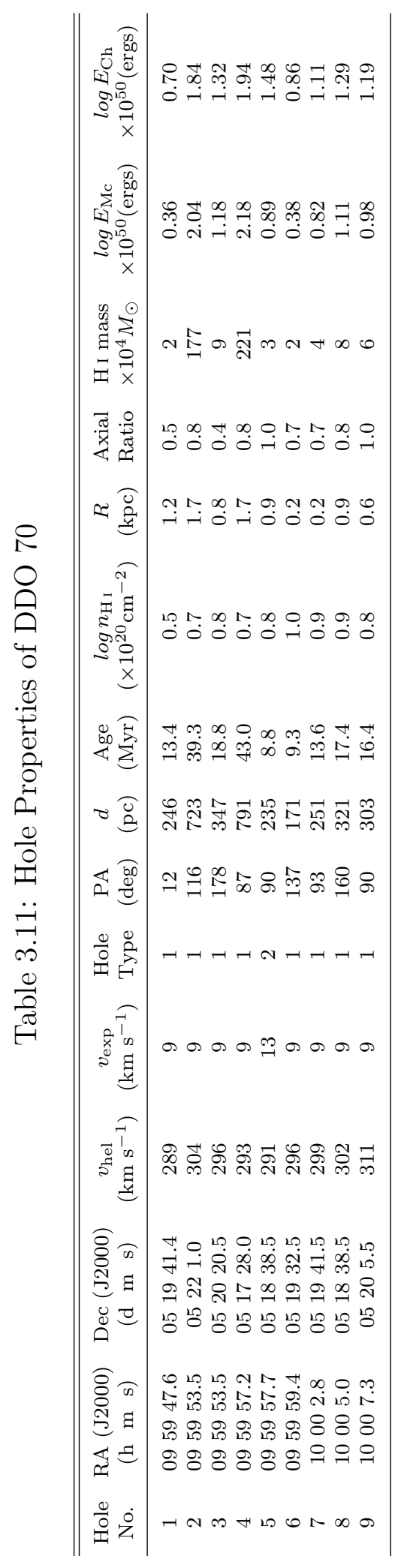




\subsubsection{DDO 75}

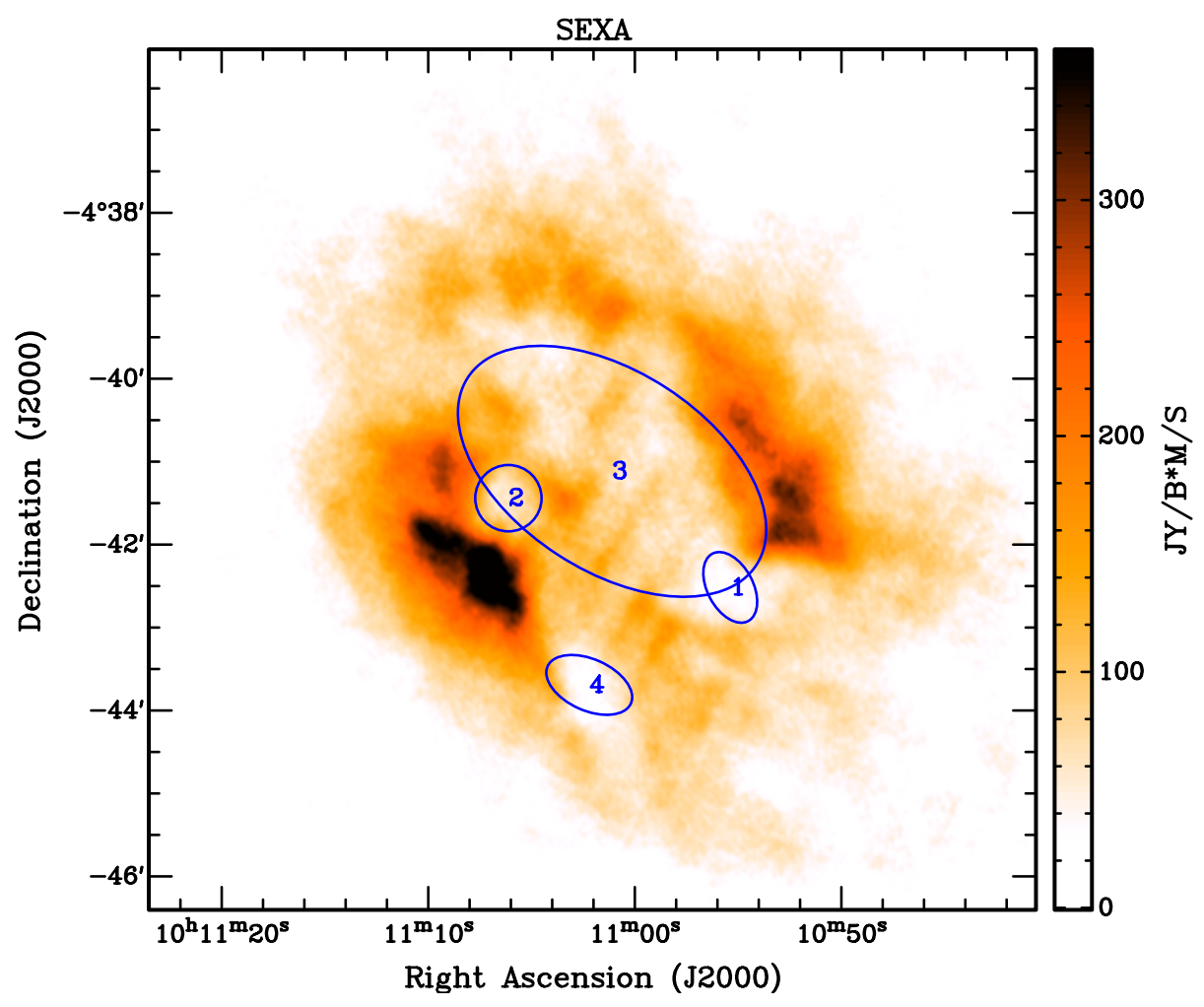

Figure 3.11: H I holes in the ISM of DDO 75 (marked with ellipses). Numbers inside the ellipse indicate the hole number from the catalog. Colors show the types of the holes: Blue ellipses are for Type 1 holes.

DDO 75, UGCA 205 or Sextans A is located at a distance of $1.3 \mathrm{Mpc}$ from the Milky Way as an isolated member of the Local Group. In V-band light it appears square shaped with magnitude -13.9. Most of the central portion of the H I disk of this galaxy is dominated by a large Type 1 hole (No. 2). It also has three smaller Type 1 holes, two of which are in the rim of the larger hole, and the last one is in the outskirt of the disk. The estimated scale height of the galaxy is $\approx 326 \mathrm{pc}$. 


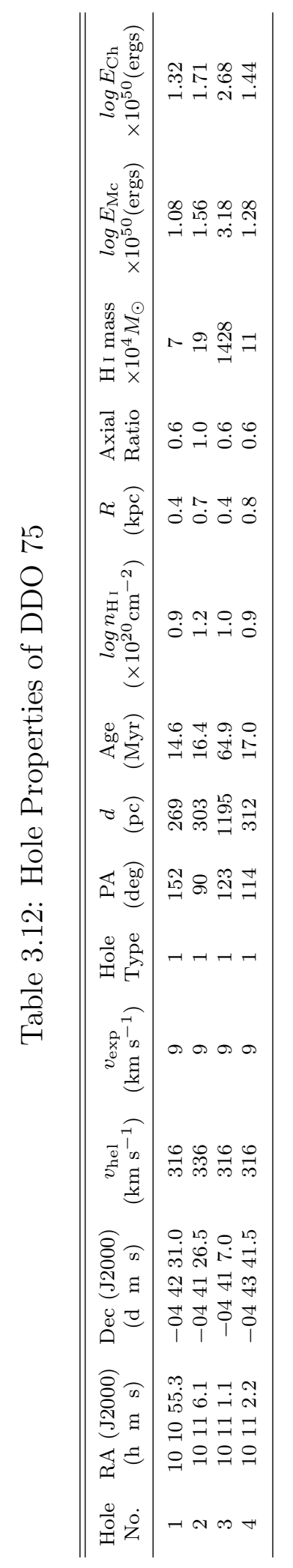




\subsubsection{DDO 87}

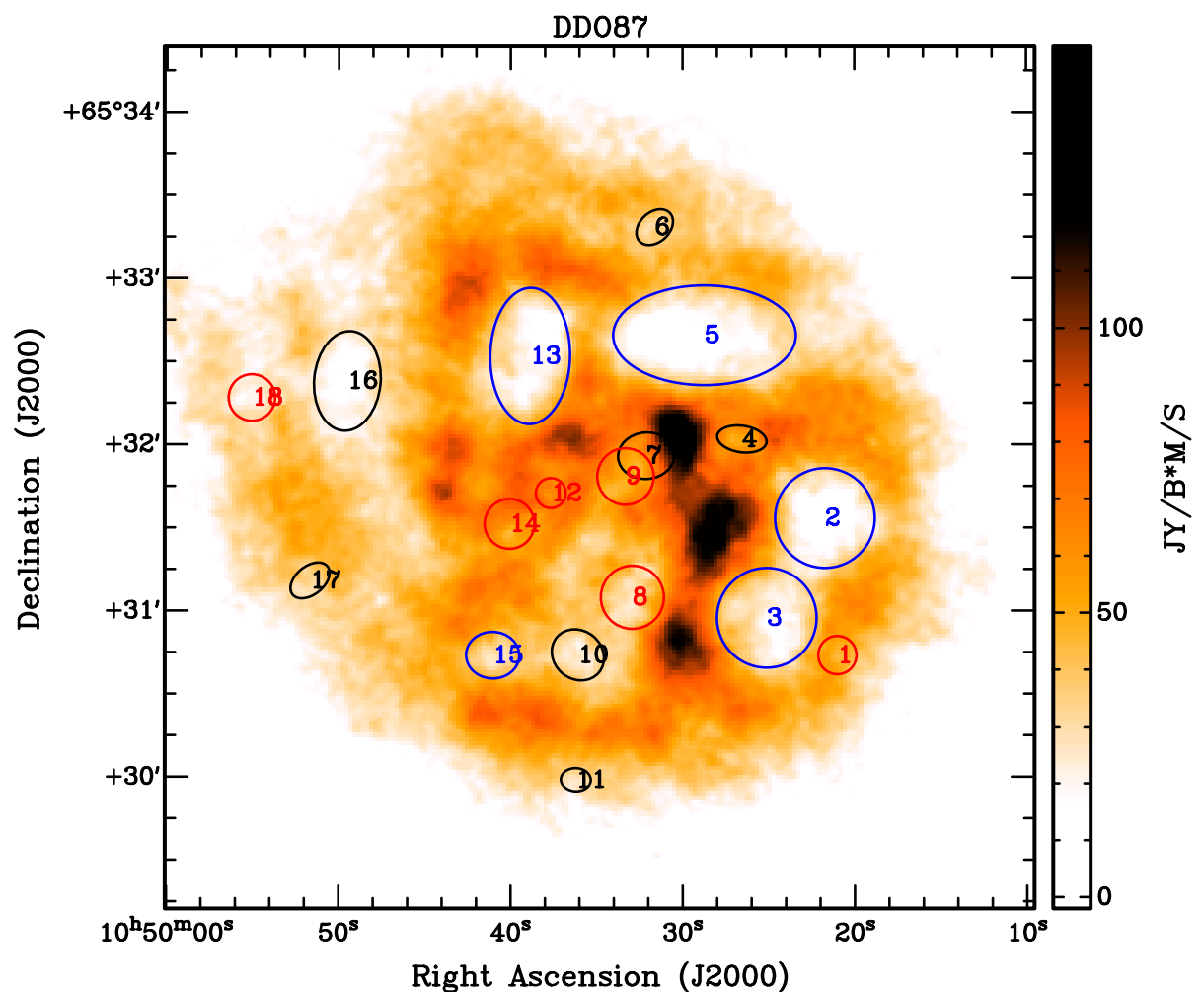

Figure 3.12: H I holes in the ISM of DDO 87 (marked with ellipses). Numbers inside the ellipse indicate the hole number from the catalog. Colors show the types of the holes: Blue ellipses are for Type 1, black are for Type 2, and red are for Type 3 holes.

DDO 87 (PGC 32405, UGC 5918) has 18 distinct H I holes in its ISM. It contains all three types of the holes almost in equal proportions. The Type 1 holes of the galaxy are larger than the others and are found in the outer part of the disk. Among them, the diameters of five of the holes are larger than one kiloparsec. The scale height of DDO 87 is estimated to be about $456 \mathrm{pc}$ and the $\mathrm{HI}$ disk radius is estimated as $7.1 \mathrm{kpc}$. This galaxy is situated about 7.7 Mpc away in the M81 Group and has a magnitude of -15 . 


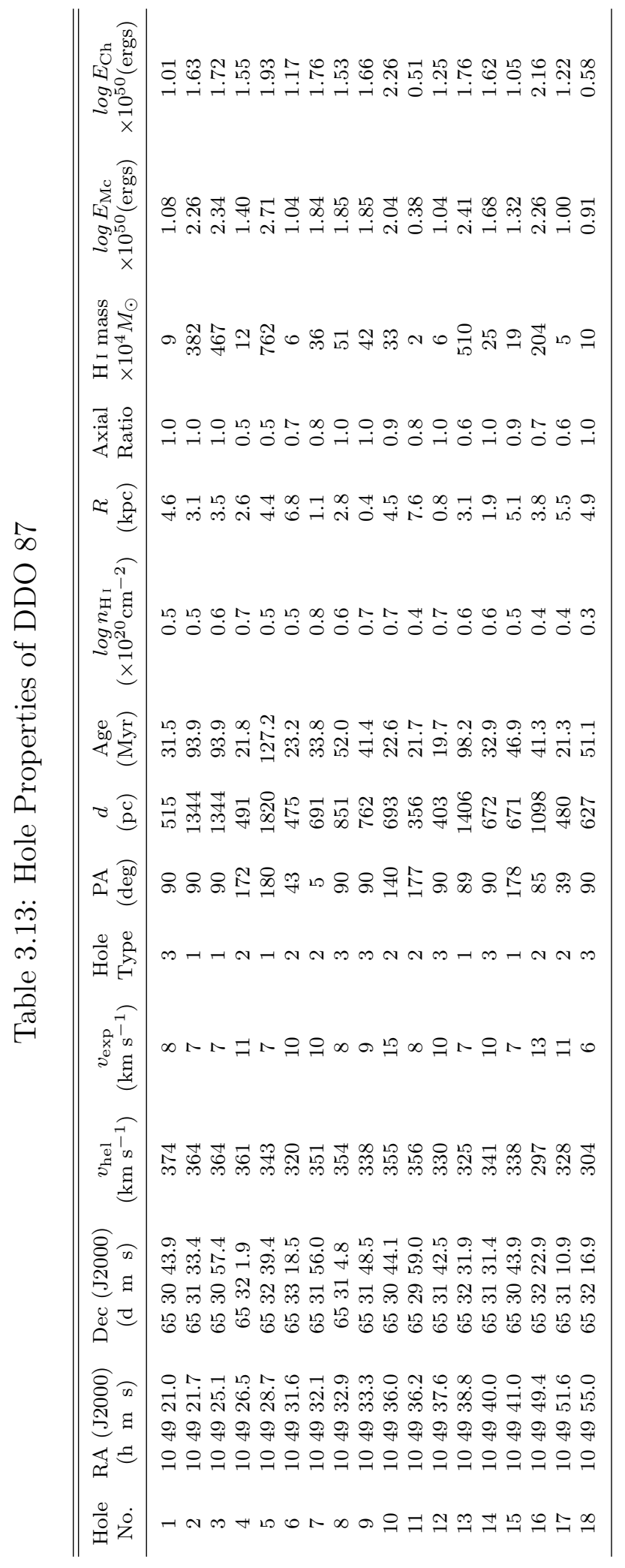




\subsubsection{DDO 101}

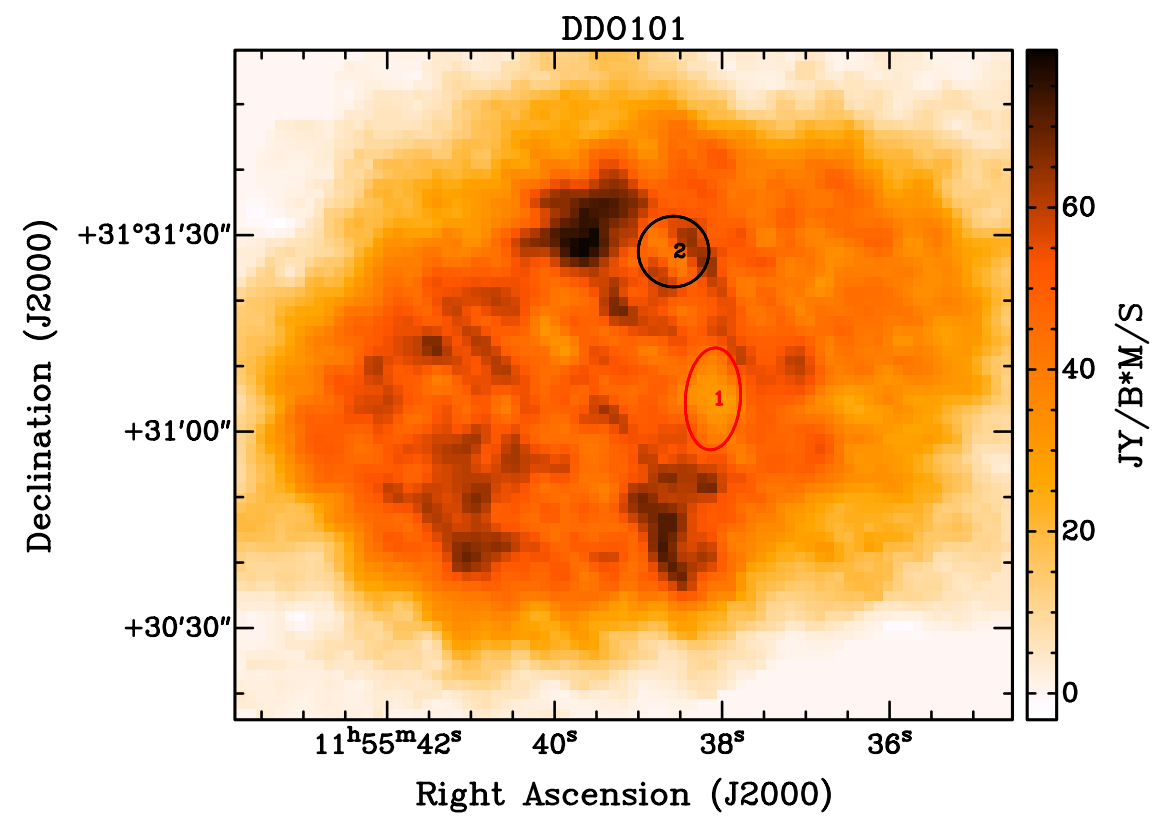

Figure 3.13: H I holes in the ISM of DDO 101 (marked with ellipses). Numbers inside the ellipse indicate the hole number from the catalog. Colors show the types of the holes: Black ellipse is for Type 2, and red is for Type 3 hole.

DDO 101 (PGC 37449, UGC 6900) is in the NGC 4062 Group at a distance of 6.4 Mpc with a magnitude of -15 . The $\mathrm{H}$ I disk of the galaxy extends to $\approx 1.8 \mathrm{kpc}$. The galaxy contains two holes, one partially broken and another intact. 


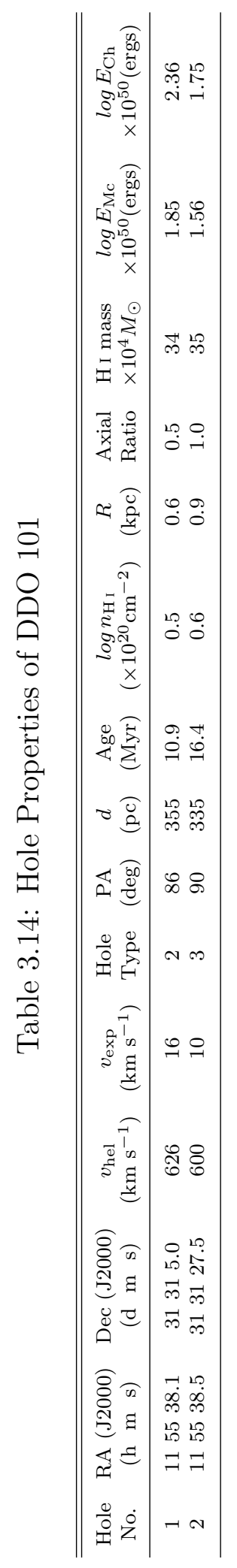




\subsubsection{DDO 126}

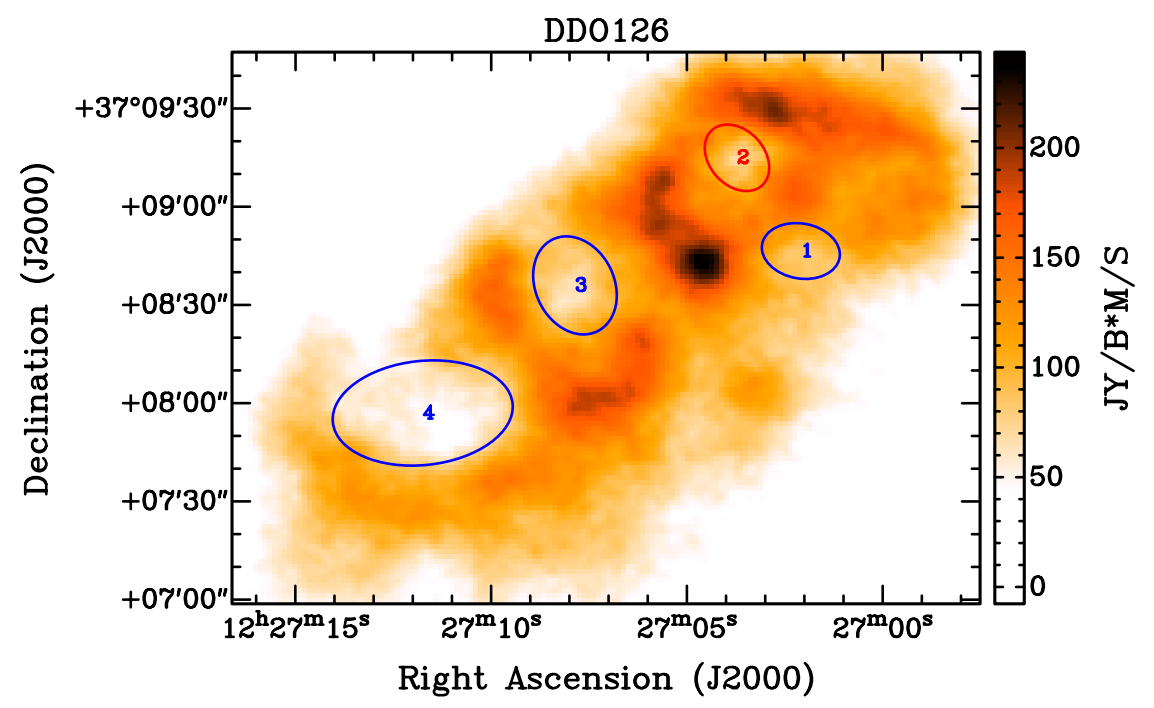

Figure 3.14: H I holes in the ISM of DDO 126 (marked with ellipses). Numbers inside the ellipse indicate the hole number from the catalog. Colors show the types of the holes: Blue ellipses are for Type 1, and red are for Type 3 holes.

DDO 126 (UGC 7559, PGC 40791) lies about 4.9 Mpc away from the Milky Way in the Canes Venatici I Group and has a V-band magnitude of -14.9. The galaxy has one Type 3 hole and three Type 1 holes including one kiloparsec-sized hole. The estimated H I disk radius and the scale height of the galaxy are about $2.9 \mathrm{kpc}$ and 294 pc respectively. 


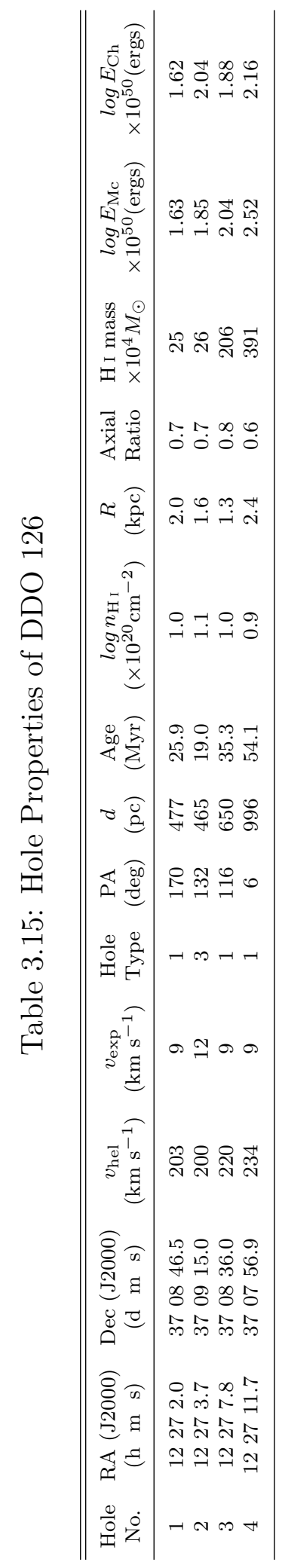




\subsubsection{DDO 133}

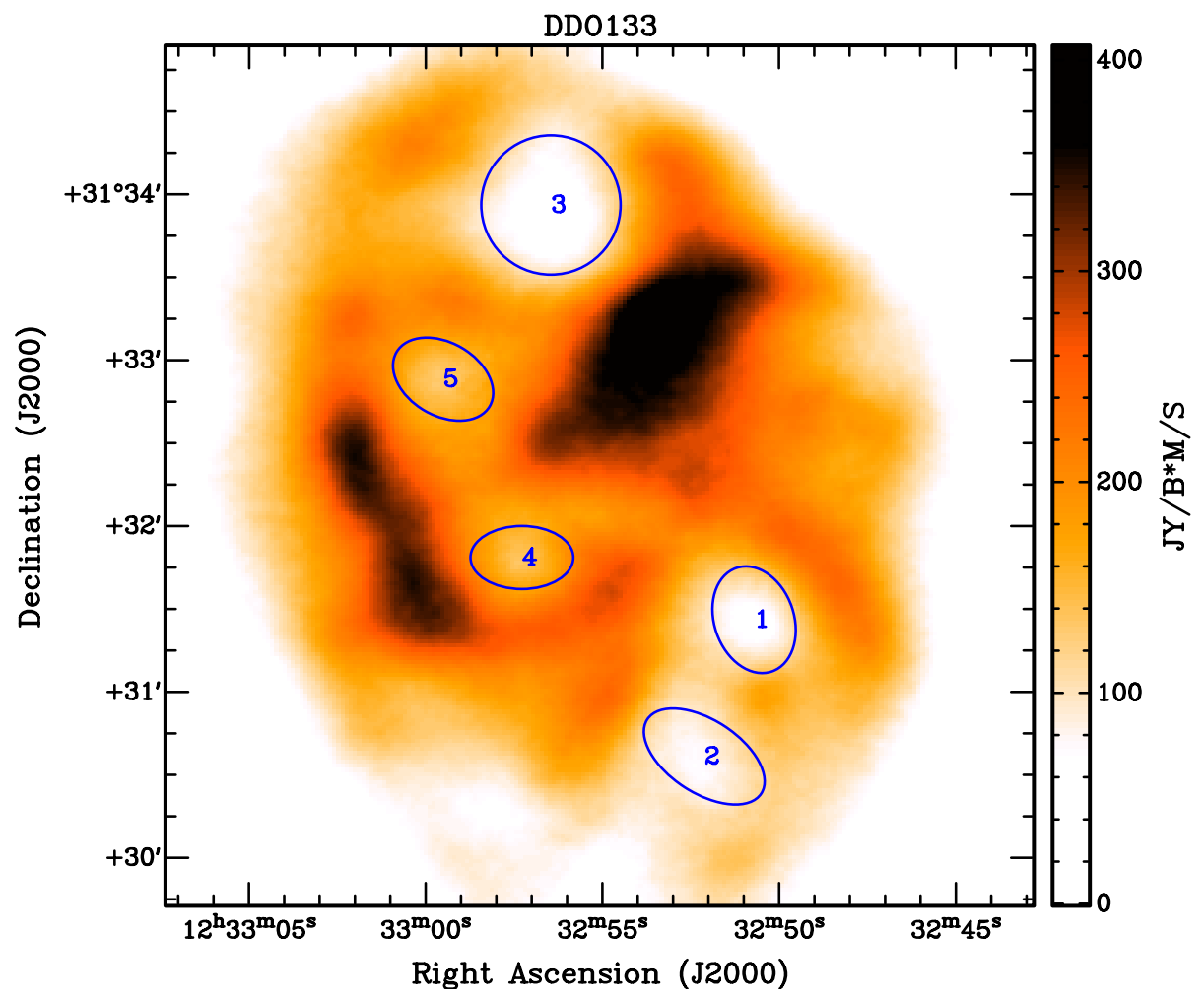

Figure 3.15: H I holes in the ISM of DDO 133 (marked with ellipses). Numbers inside the ellipse indicate the hole number from the catalog. Colors show the types of the holes: Blue ellipses are for Type 1 holes.

DDO 133 (PGC 41636, UGC 76980) is situated at a distance of $3.5 \mathrm{Mpc}$ in the Canes Venatici I Group. Its V-band magnitude is -14.8. DDO 133 has five holes in the ISM, and all are Type 1 holes. 


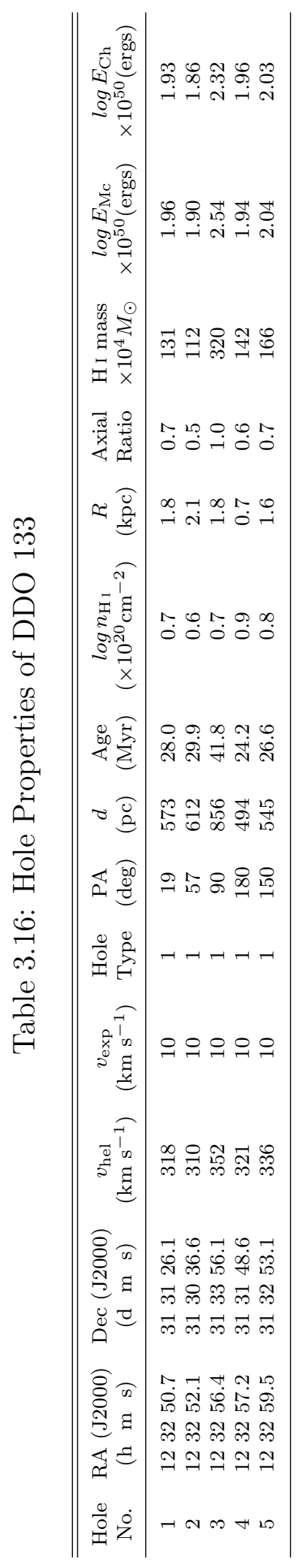




\subsubsection{DDO 154}

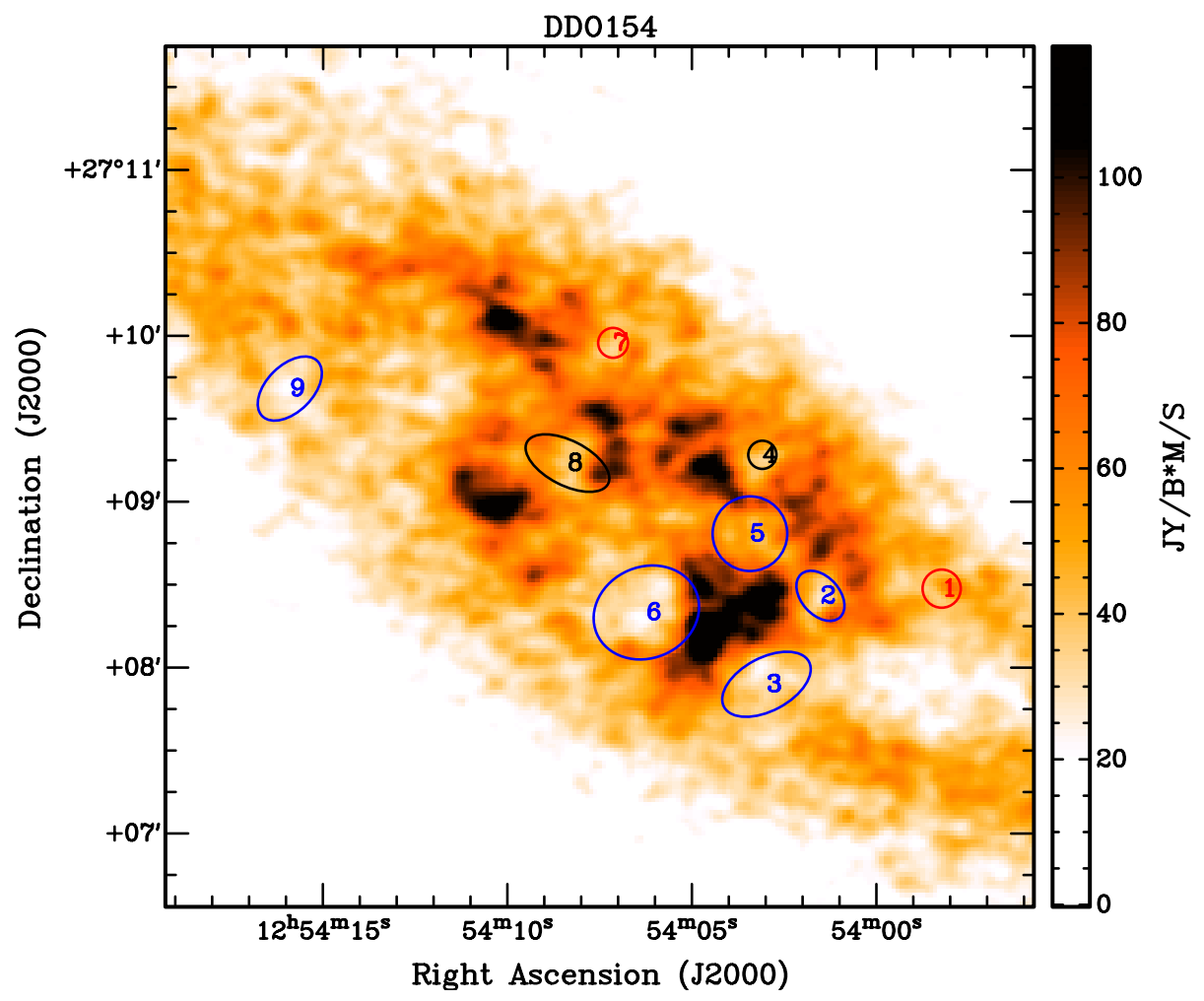

Figure 3.16: H I holes in the ISM of DDO 154 (marked with ellipses). Numbers inside the ellipse indicate the hole number from the catalog. Colors show the types of the holes: Blue ellipses are for Type 1, black are for Type 2, and red are for Type 3 holes.

DDO 154, also known as PGC 43869, UGC 8204 and NGC 4789A, is $3.7 \mathrm{Mpc}$ away from us with a magnitude of -14.2 in the Canes Venatici I Group [97]. An earlier study done by Hoffman et al. [98] found two holes and Bagetakos et al. [33] found nine holes. We also detected nine holes with the majority being of Type 1. Among LITTLE THINGS galaxies, DDO 154 has one of the largest H I disk sizes $\left(R_{\max }=\right.$ $7.1 \mathrm{kpc})$. 


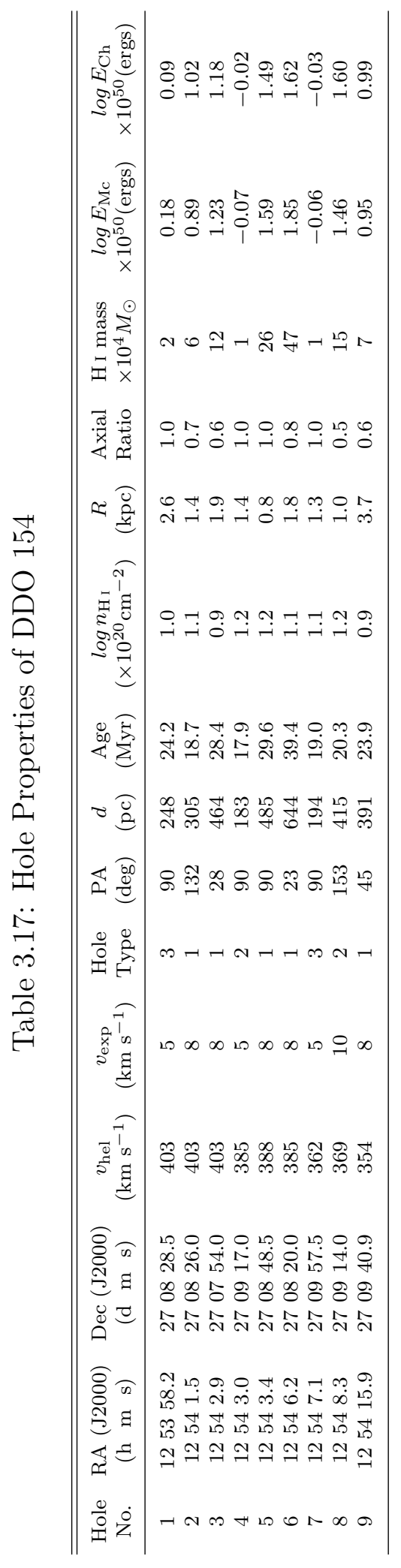




\subsubsection{DDO 155}

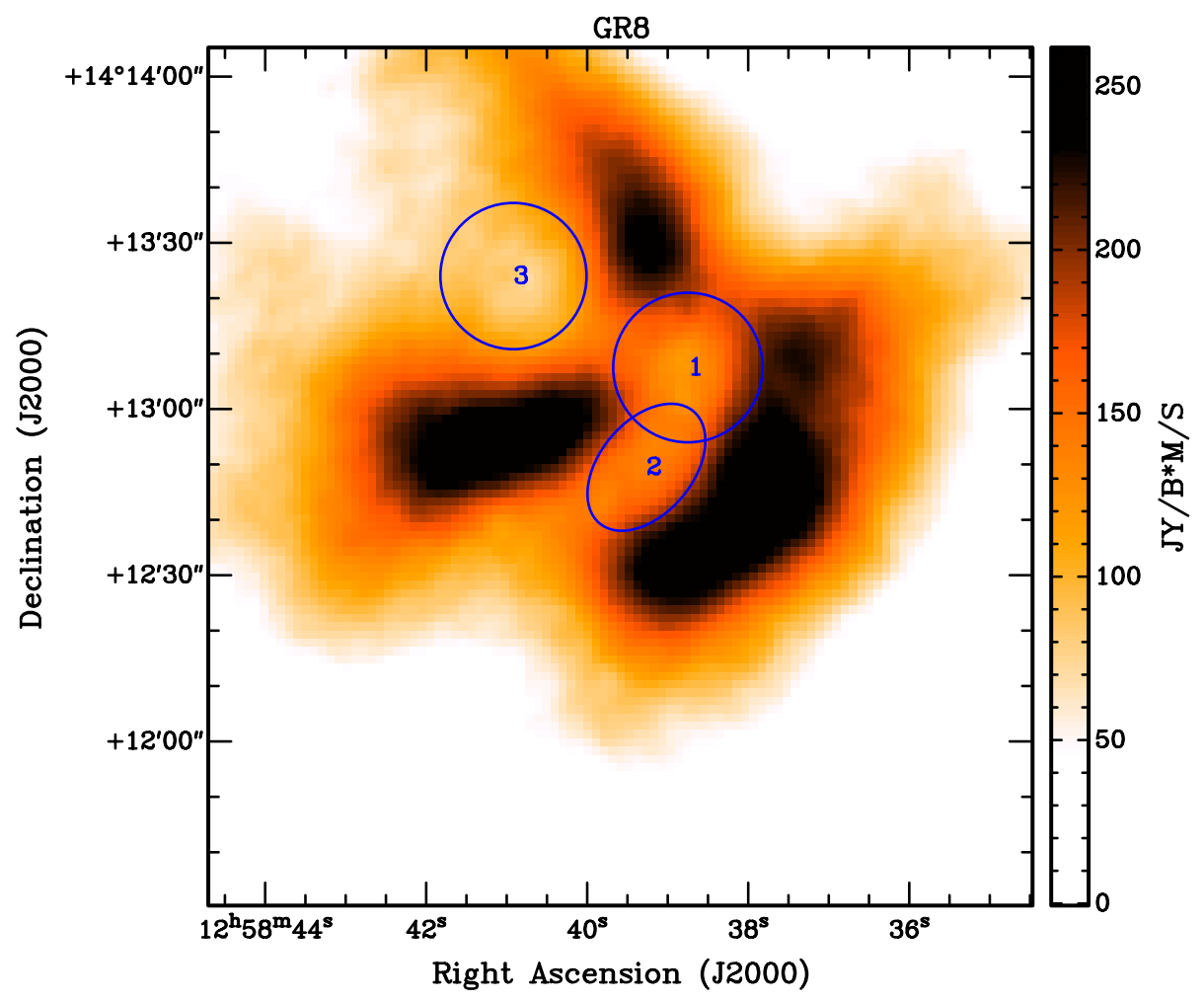

Figure 3.17: H I holes in the ISM of DDO 155 (marked with ellipses). Numbers inside the ellipse indicate the hole number from the catalog. Colors show the types of the holes: Blue ellipses are for Type 1 holes.

DDO 155, Gr 8, LSBC D646-07, PGC 44491 or UGC 8091 has three completely broken holes in its $\mathrm{H}$ I disk with two of them overlapping. We estimated the scale height of the galaxy about $141 \mathrm{pc}$ and the disk radius about $0.7 \mathrm{kpc}$. This galaxy is located in the Local Group at a distance of about $2.2 \mathrm{Mpc}$. 


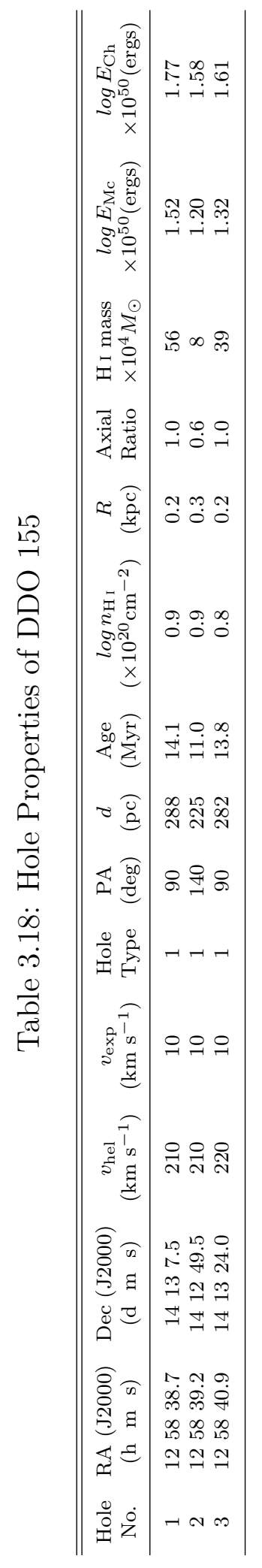




\subsubsection{DDO 165}

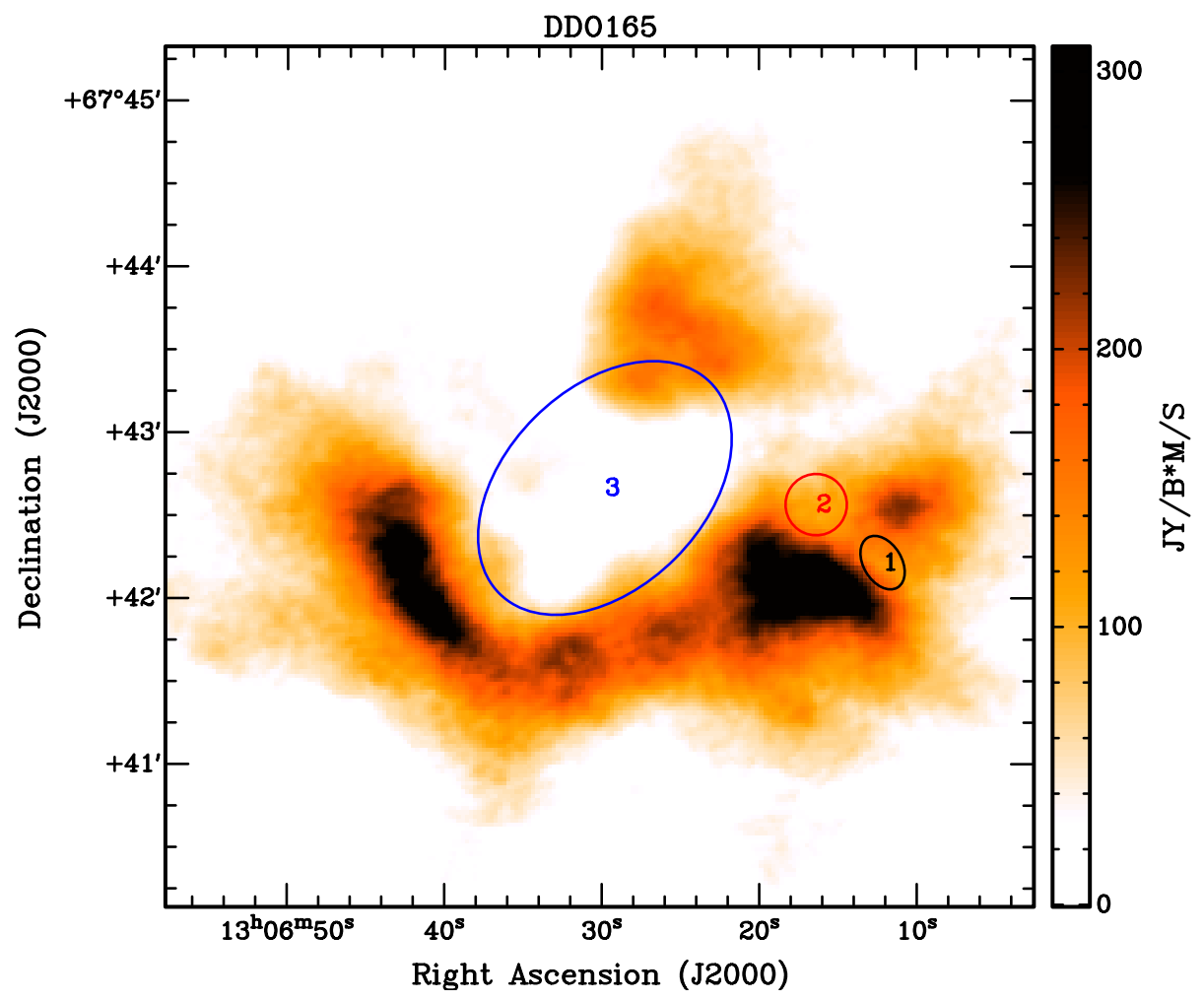

Figure 3.18: H I holes in the ISM of DDO 165 (marked with ellipses). Numbers inside the ellipse indicate the hole number from the catalog. Colors show the types of the holes: Blue ellipse is for Type 1, black is for Type 2, and red is for Type 3 hole.

DDO 165 (Mailyan 82, PGC 45372 or UGC 8201) is an M81 Group dwarf at a distance of 4.6 Mpc with a magnitude of -15.6. Cannon et al. [61] detected seven H i holes in DDO 165. According to our criteria, we have only three 'good quality' holes, one of each type. The largest hole we found is in agreement with Cannon et al. [61] whereas the other two holes are listed by them as a single hole. 


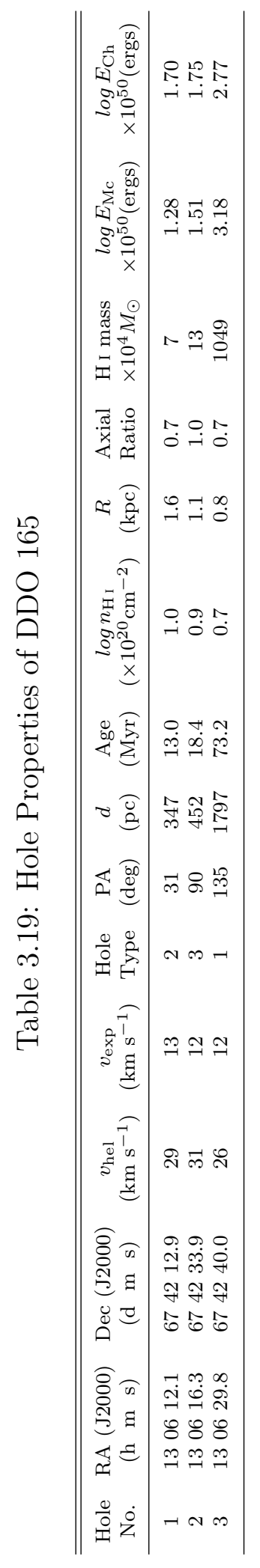




\subsubsection{DDO 167}

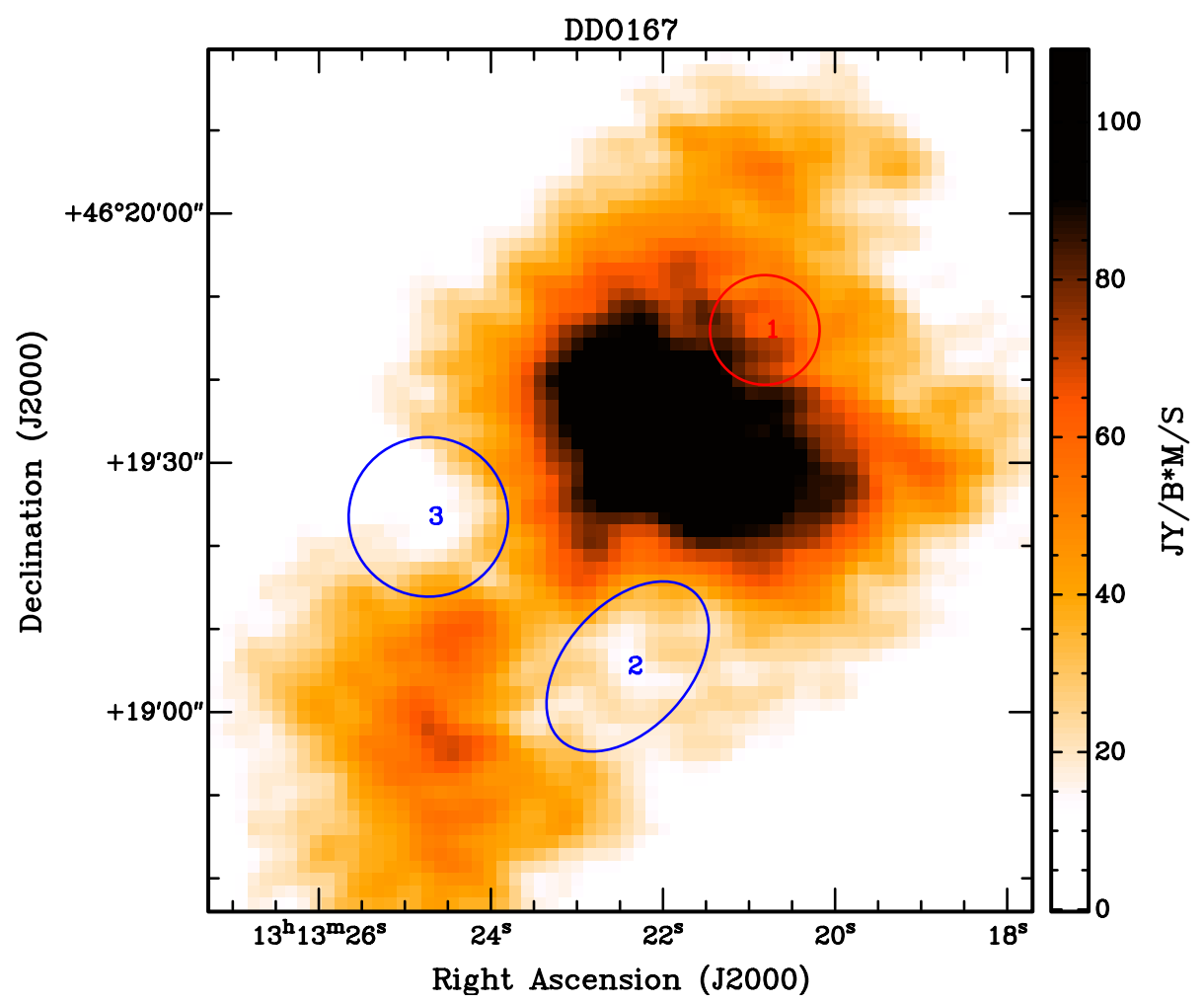

Figure 3.19: H I holes in the ISM of DDO 167 (marked with ellipses). Numbers inside the ellipse indicate the hole number from the catalog. Colors show the types of the holes: Blue ellipses are for Type 1, and red are for Type 3 holes.

DDO 167, also known as PGC 45939 or UGC 8308 lies in the Canes Venatici I group about 4.2 Mpc away. The galaxy has two Type 1 holes and a Type 3 hole in the H I disk which extends to $\approx 1 \mathrm{kpc}$. The estimated $\mathrm{H}$ I scale height of the galaxy is about 237 pc. 


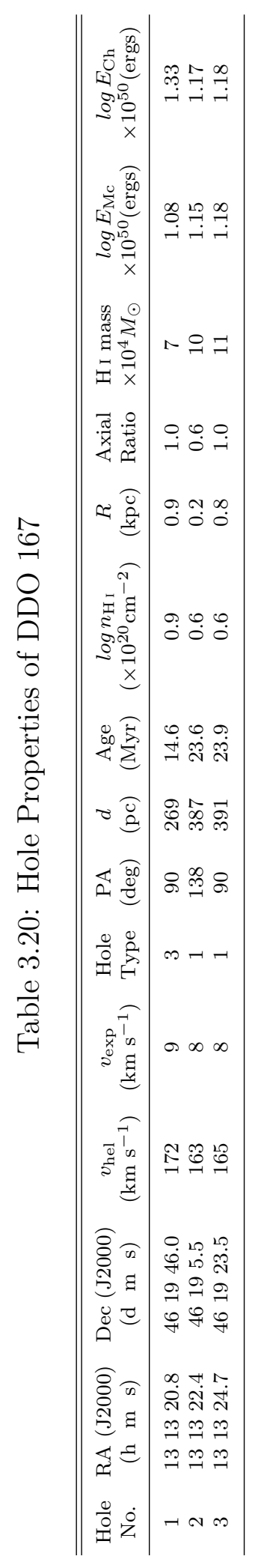




\subsubsection{DDO 168}

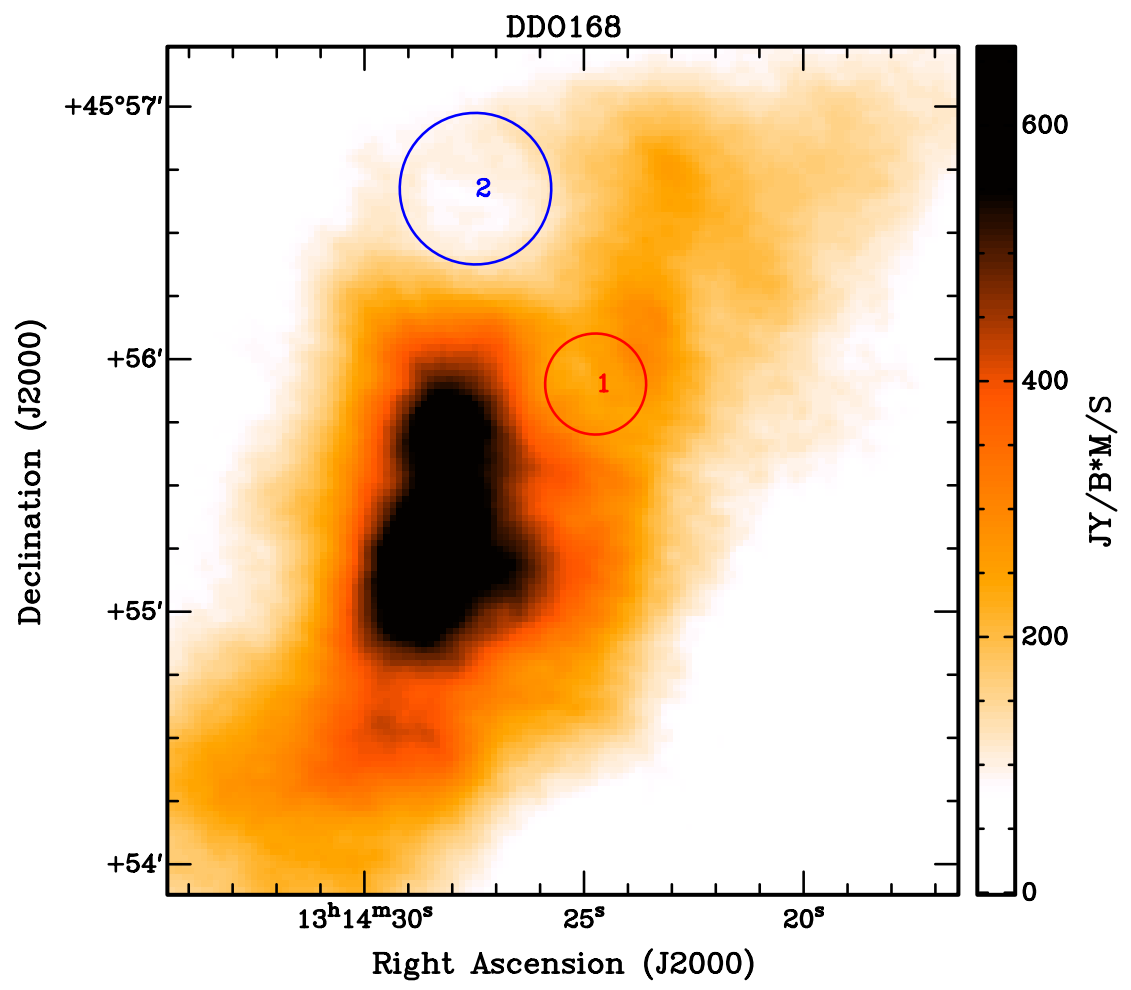

Figure 3.20: H I holes in the ISM of DDO 168 (marked with ellipses). Numbers inside the ellipse indicate the hole number from the catalog. Colors show the types of the holes: Blue ellipse is for Type 1, and red is for Type 3 hole.

DDO 168 (PGC 46039, UGC 8320) is another Canes Venatici I group member also known as PGC 46039 and UGC 8320. It is located $4.3 \mathrm{Mpc}$ away with magnitude -15.7. The galaxy has two holes, both located in the northern part of the H I disk. The H I scale height of the galaxy is estimated as 206 pc. 


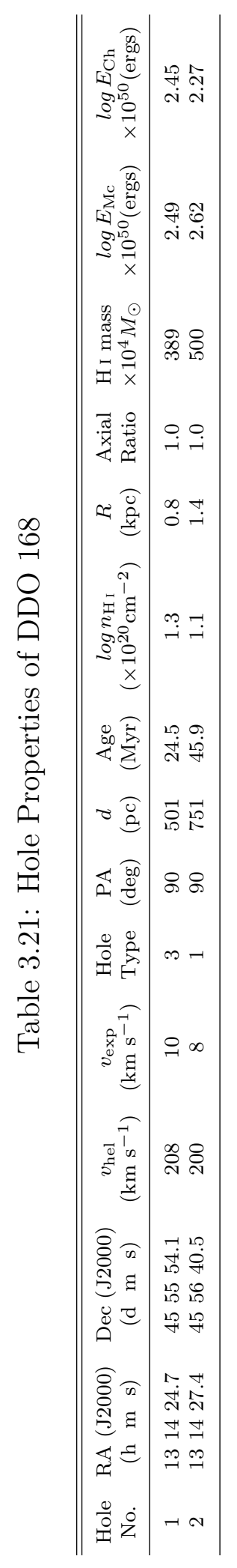




\subsubsection{DDO 187}

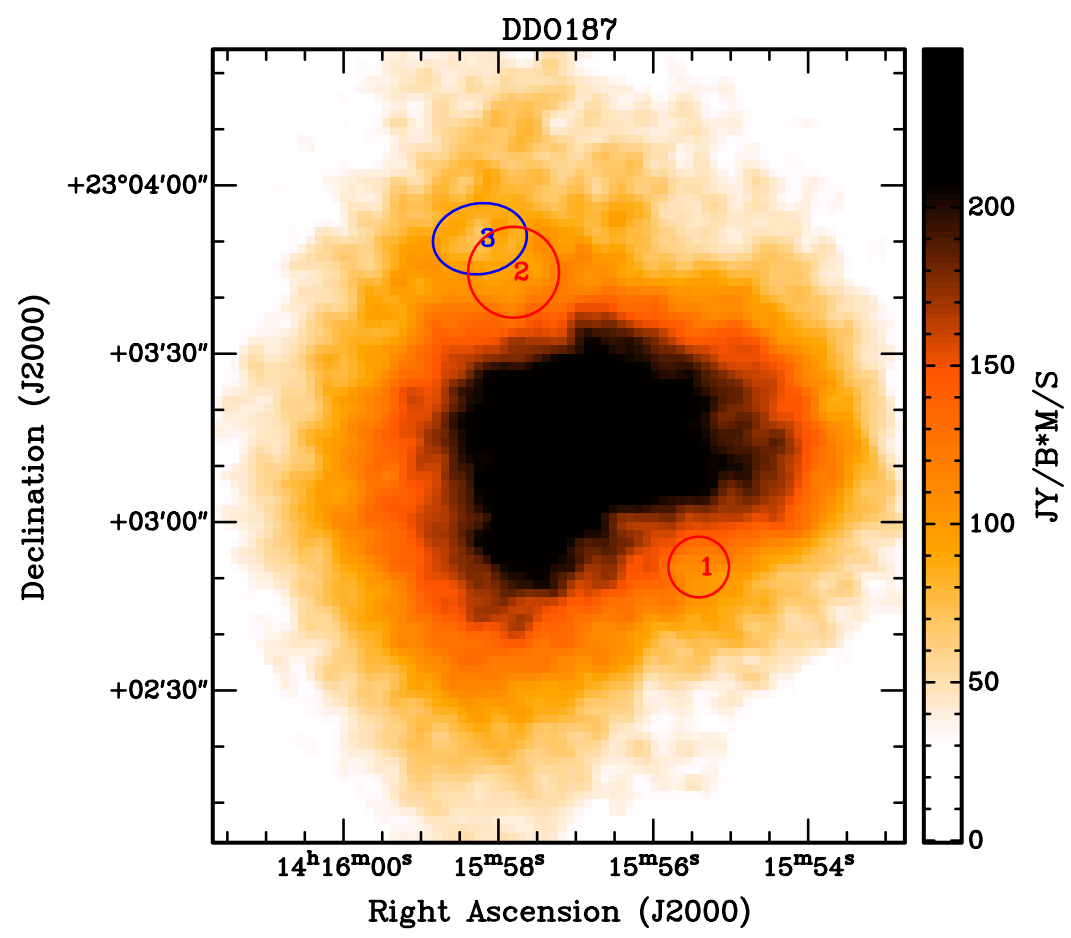

Figure 3.21: H I holes in the ISM of DDO 187 (marked with ellipses). Numbers inside the ellipse indicate the hole number from the catalog. Colors show the types of the holes: Blue ellipses are for Type 1, and red are for Type 3 holes.

DDO 187 (PGC 50961, UGC 9128) is one of the smallest galaxy in the sample in terms of $\mathrm{H}$ I disk radius. It has three holes and two of them (Nos. 2 and 3) are overlapped. The galaxy is at a distance of $2.2 \mathrm{Mpc}$ from the Milky Way with a magnitude of -12.7. We estimated the scale height and the size of the H I disk to be about $0.5 \mathrm{kpc}$ and 64 pc respectively. 


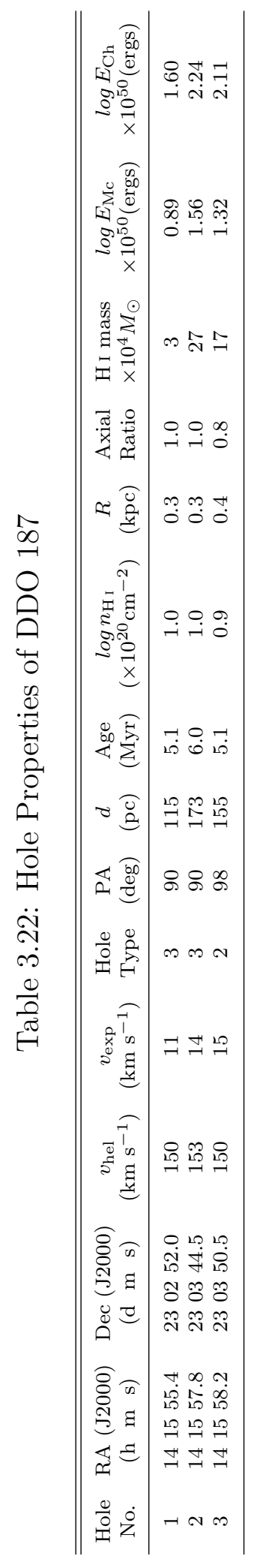




\subsubsection{DDO 210}

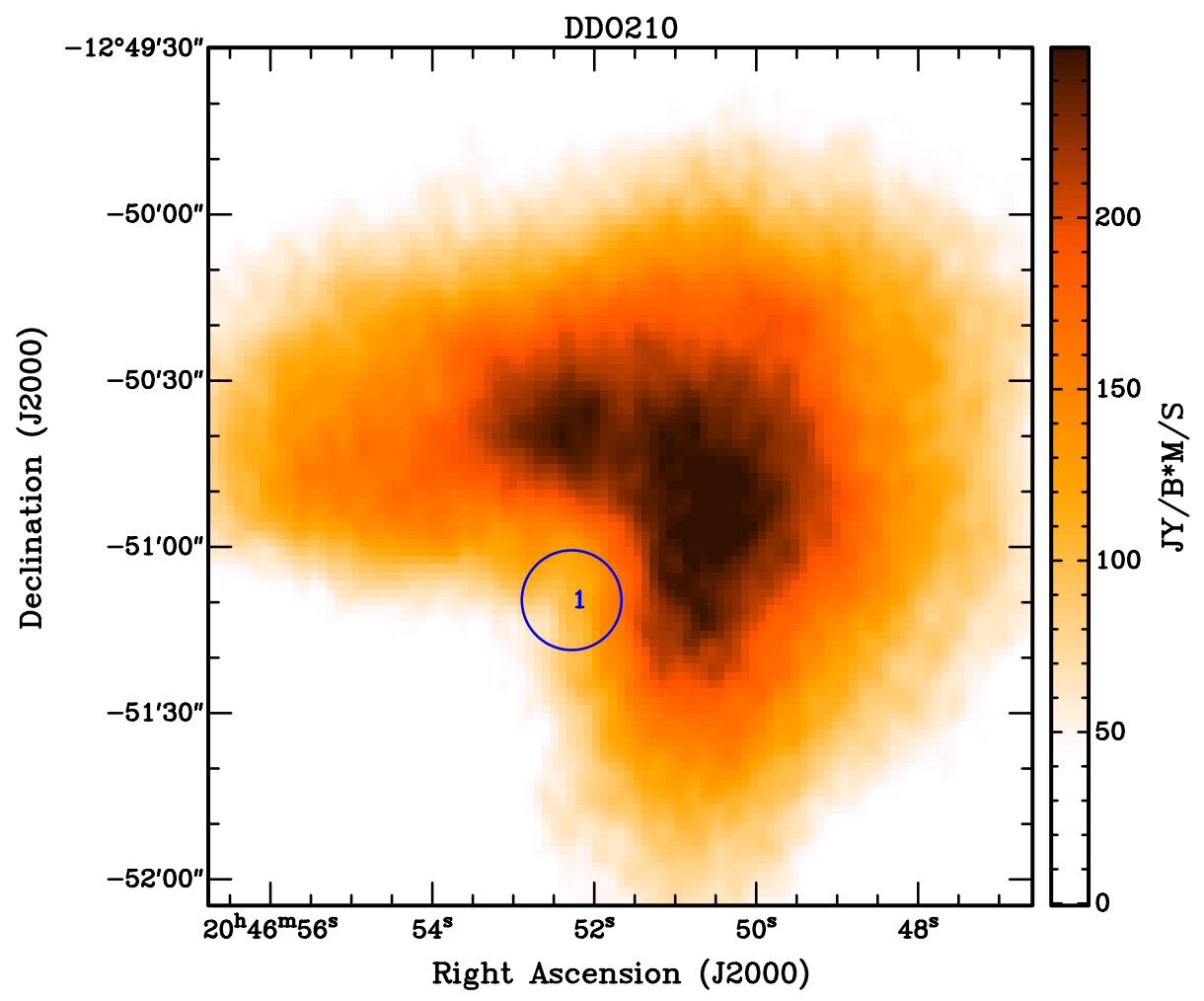

Figure 3.22: H I hole in the ISM of DDO 210 (marked with ellipse). Number inside the ellipse indicates the hole number from the catalog. Blue ellipse is for Type 1 hole.

DDO 210 is also known as PGC 65367 or the Aquarius Dwarf. It is a relatively isolated member of Local Group situated about 0.9 Mpc away with a V-band magnitude of -10.9. The galaxy has one small Type 1 hole of of diameter $\approx 79$ pc. We estimated the scale height about $113 \mathrm{pc}$ and the rotational velocity about $11 \mathrm{~km} \mathrm{~s}^{-1}$. 


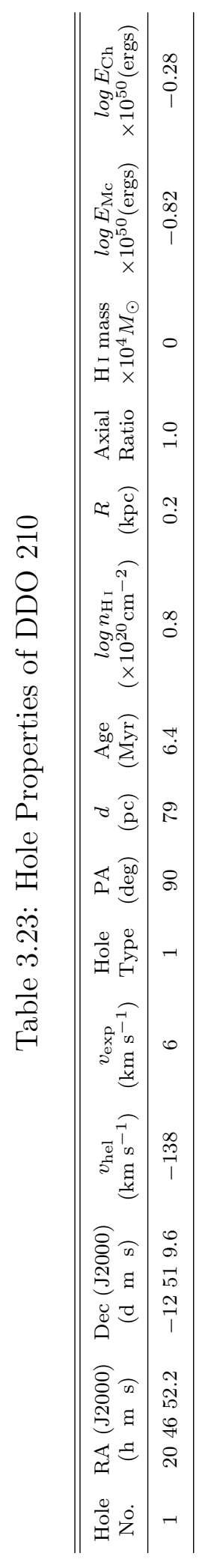




\subsubsection{DDO 216}

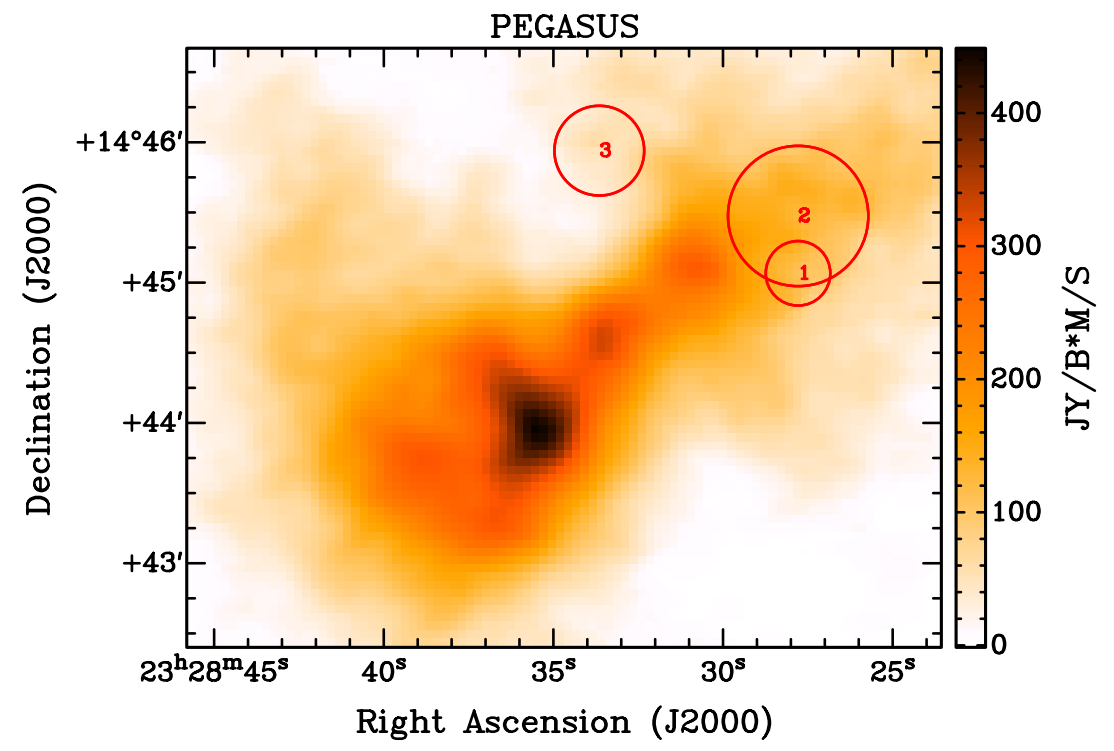

Figure 3.23: H I holes in the ISM of DDO 216 (marked with ellipses). Numbers inside the ellipse indicate the hole number from the catalog. Colors show the types of the holes: Red ellipses are for Type 3 holes.

DDO 216 (Peg DIG, Pegasus Dwarf, UGC 12613 or PGC 71538) is another Local Group dwarf at a distance of $1.1 \mathrm{Mpc}$. The galaxy is a companion of Andromeda and lies in the Pegasus constellation. It is one of the smallest galaxies in the LITTLE THINGS group with H I disk radius about $0.7 \mathrm{kpc}$. The galaxy has three Type 3 holes. 


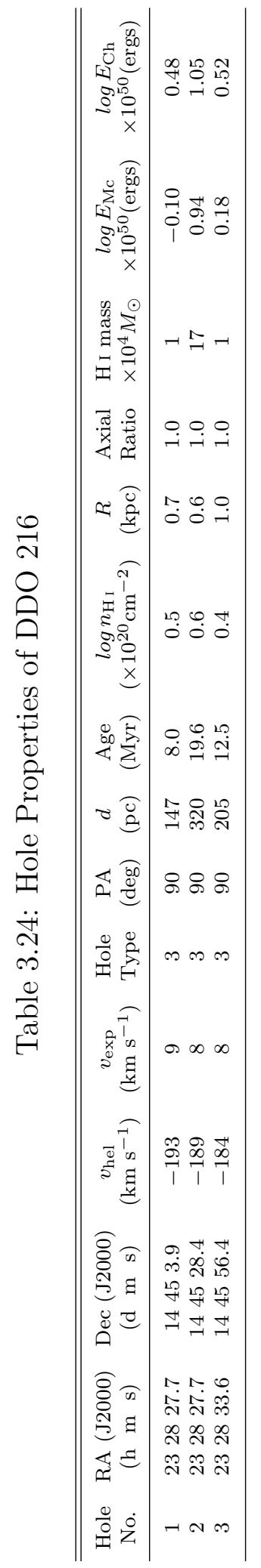




\subsubsection{F564-V3}

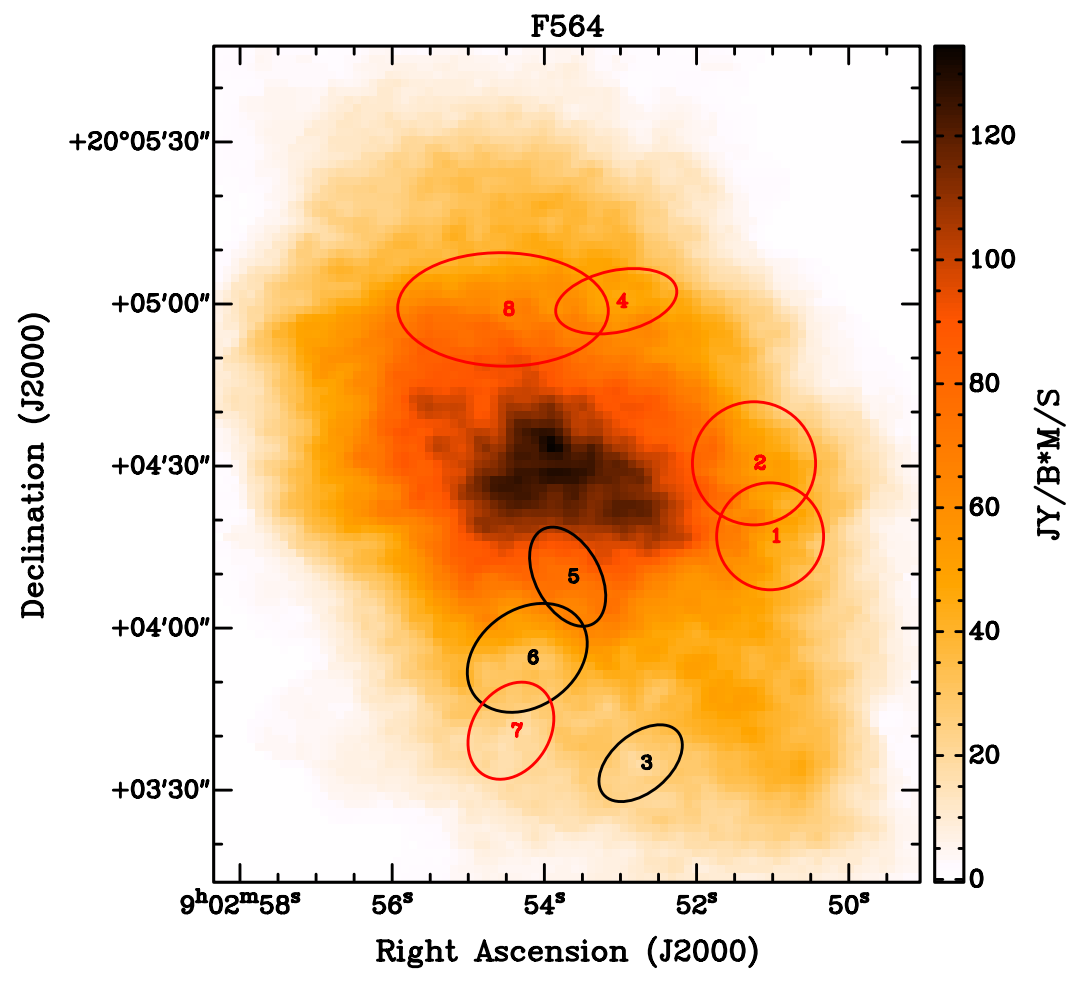

Figure 3.24: H I holes in the ISM of F564-V3 (marked with ellipses). Numbers inside the ellipse indicate the hole number from the catalog. Colors show the types of the holes: Black ellipses are for Type 2, and red are for Type 3 holes.

F564-V3 or LSBC D564-08 is situated at a distance of about 8.7 Mpc away from the Milky Way with V-band surface brightness -14. We detected eight H I holes in this galaxy, five of which are Type 3 and the rest of which are Type 2. The scale height of the galaxy is $\approx 136 \mathrm{kpc}$ and the $\mathrm{H}$ I disk is extended to about $2.4 \mathrm{kpc}$. 


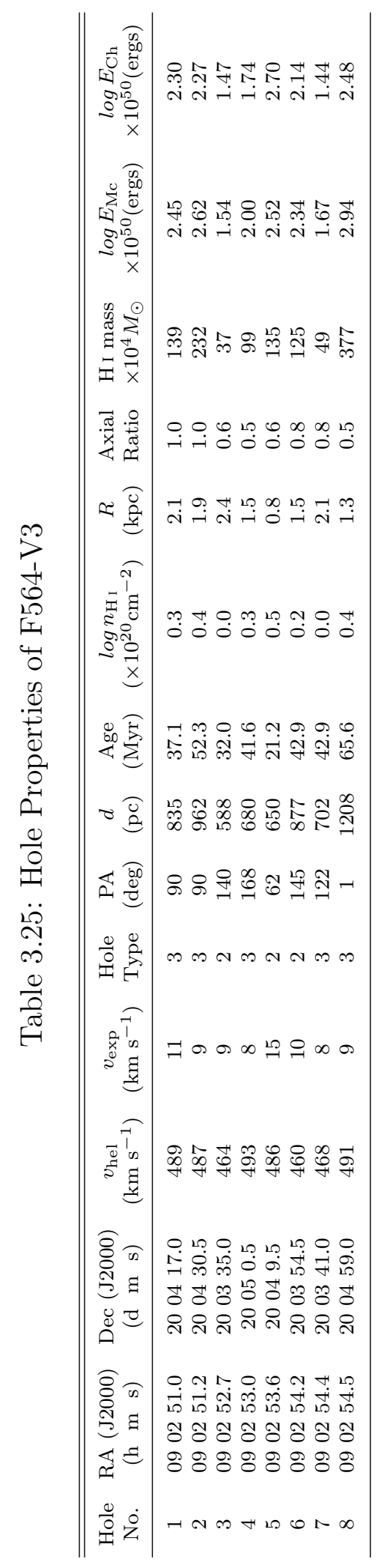




\subsubsection{Haro 29}

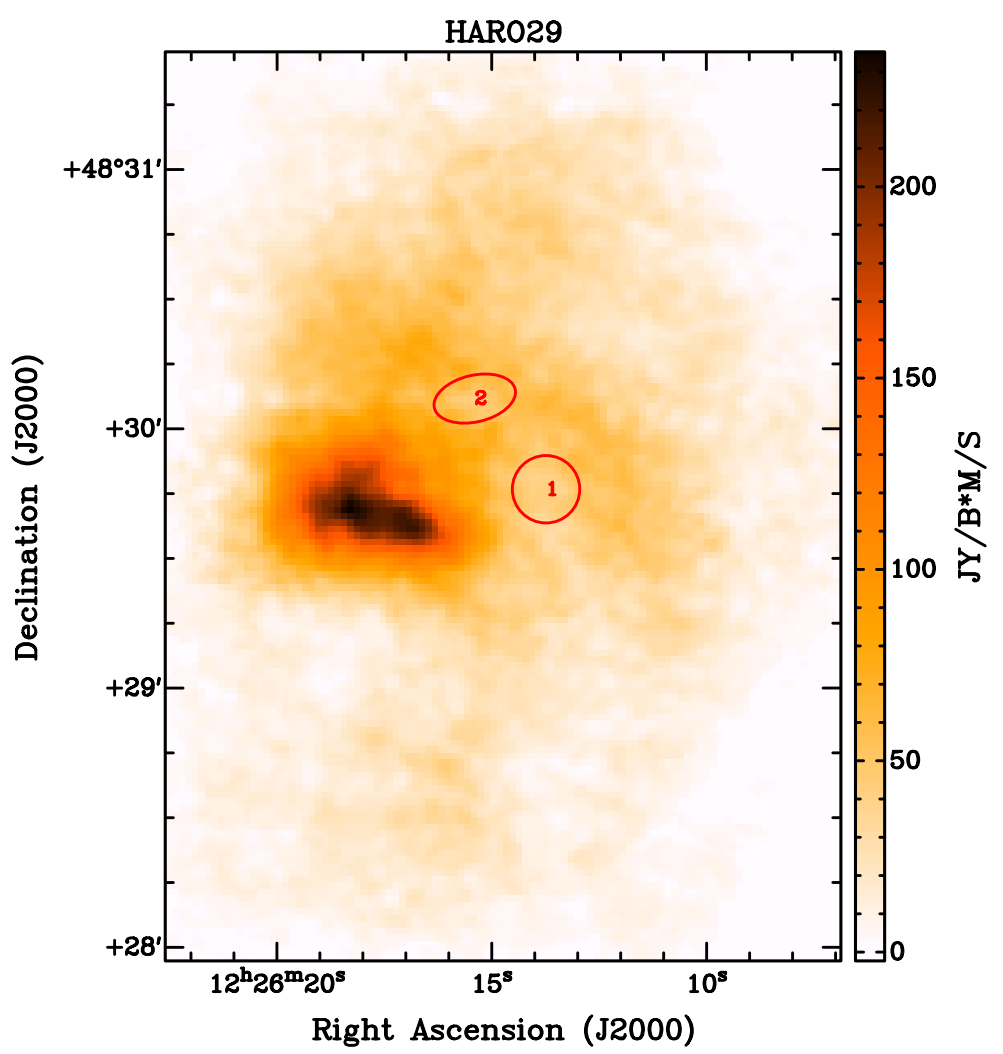

Figure 3.25: H I holes in the ISM of Haro 29 (marked with ellipses). Numbers inside the ellipse indicate the hole number from the catalog. Red ellipses are for Type 3 holes.

Haro 29 is a starburst blue compact dwarf. It is a member of the Canes Venatici I Group of galaxies [97] and is at a distance of about $5.8 \mathrm{Mpc}$. It is also known as Mrk 209, I Zw 36, UGCA 281 and PGC 40665. The H I scale height of this galaxy is 222 $\mathrm{pc}$ and the $\mathrm{H}$ I disk radius is $\approx 2.3 \mathrm{kpc}$ respectively. The galaxy has two intact holes. 


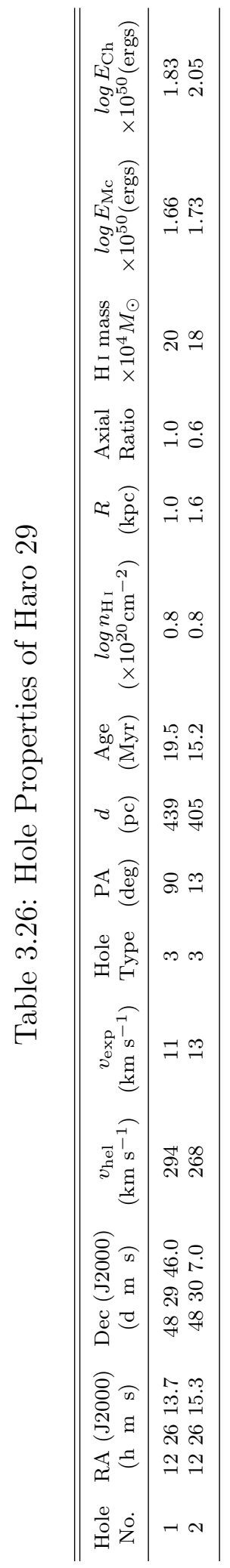




\subsubsection{Haro 36}

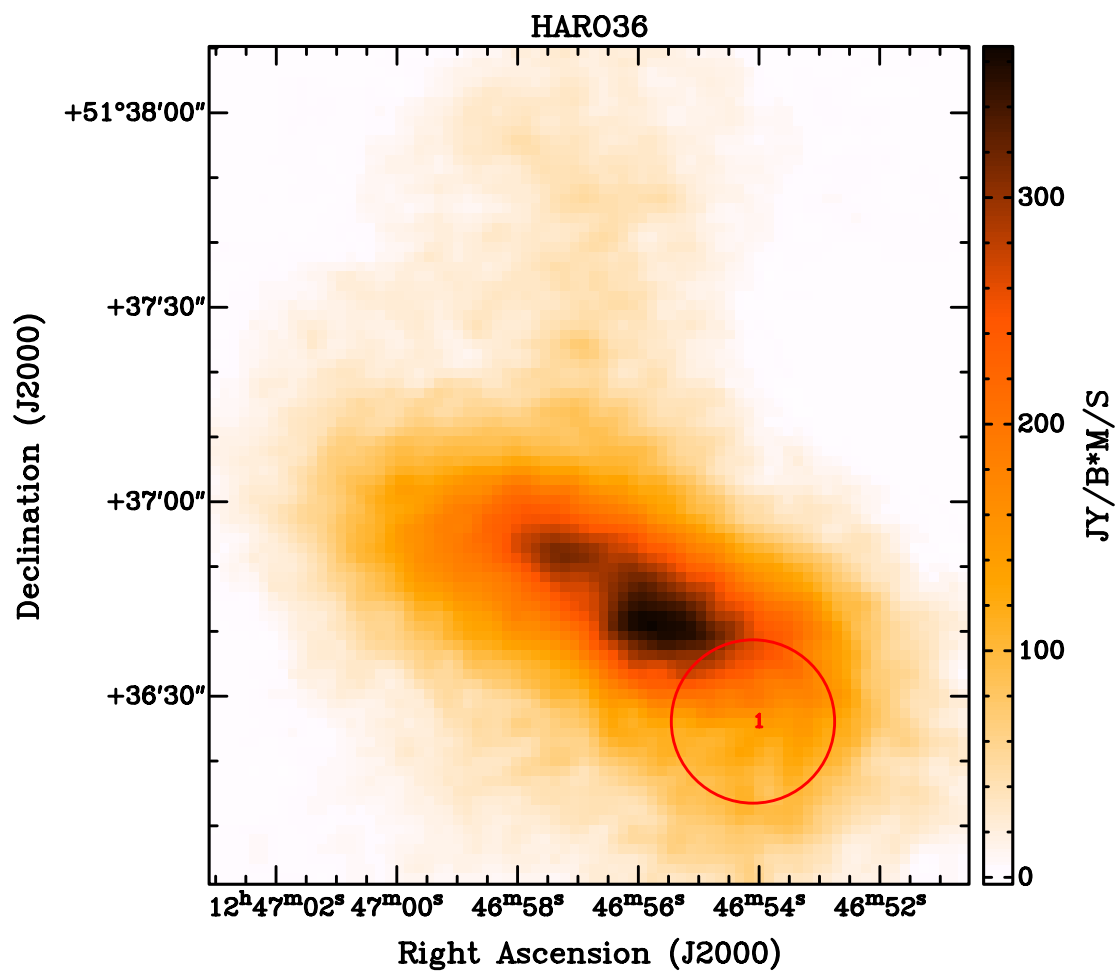

Figure 3.26: H I hole in the ISM of Haro 36 (marked with ellipse). Number inside the ellipse indicates the hole number from the catalog. Red ellipse is for Type 3 hole.

Haro 36 (PGC 43124, UGC 7950) is located about $9.3 \mathrm{kpc}$ away and is the second most distant galaxy in the sample. It is a galaxy with a kiloparsec-sized Type 3 hole with expansion velocity $18 \mathrm{~km} \mathrm{~s}^{-1}$. Interestingly, the size of this intact hole is larger than the galaxy's scale height of 220 pc, which suggests that the hole might not be completely spherical in nature. The rotational velocity of the galaxy is $\approx 76 \mathrm{~km} \mathrm{~s}^{-1}$. 


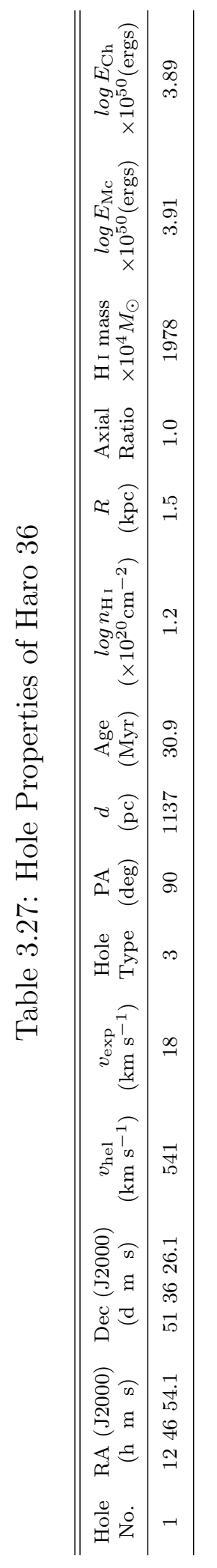




\subsubsection{IC 10}

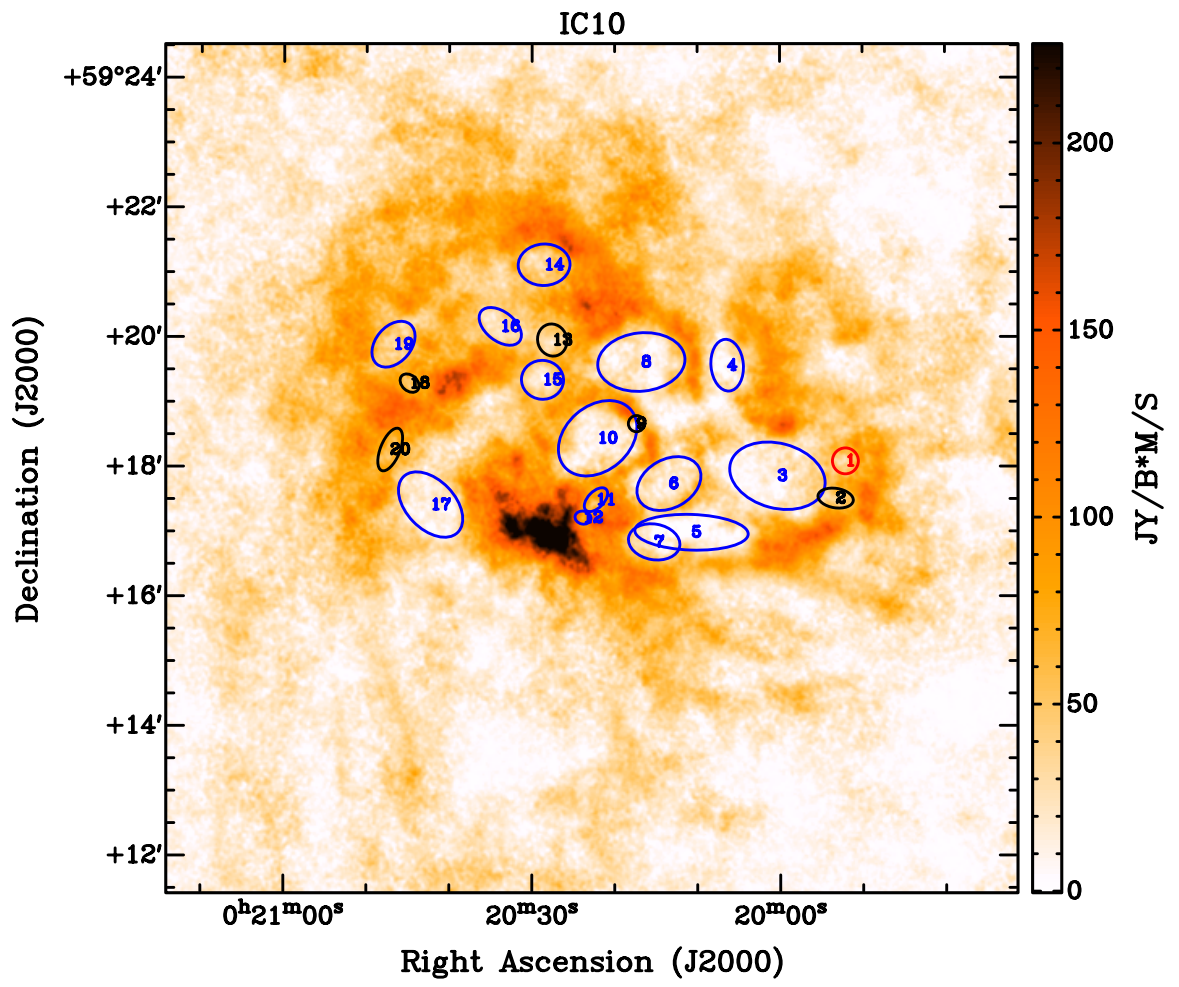

Figure 3.27: H I holes in the ISM of IC 10 (marked with ellipses). Numbers inside the ellipse indicate the hole number from the catalog. Colors show the types of the holes: Blue ellipses are for Type 1 holes.

IC 10, PGC 1305, or UGC 192 is a well-studied Local Group blue compact dwarf galaxy. It is located in the constellation Cassiopeia, at a distance of $0.7 \mathrm{Mpc}$, with magnitude -16.3. Wilcots and Miller [99] first studied the holes in IC 10 and found eight holes. Based on our criteria, we detected 20 holes, most of which are Type 1. Our analysis shows that the large holes discovered by Wilcots and Miller [99] contain two or more smaller holes and some of them are overlapped. We estimated the scale height of the galaxy as $189 \mathrm{pc}$. 


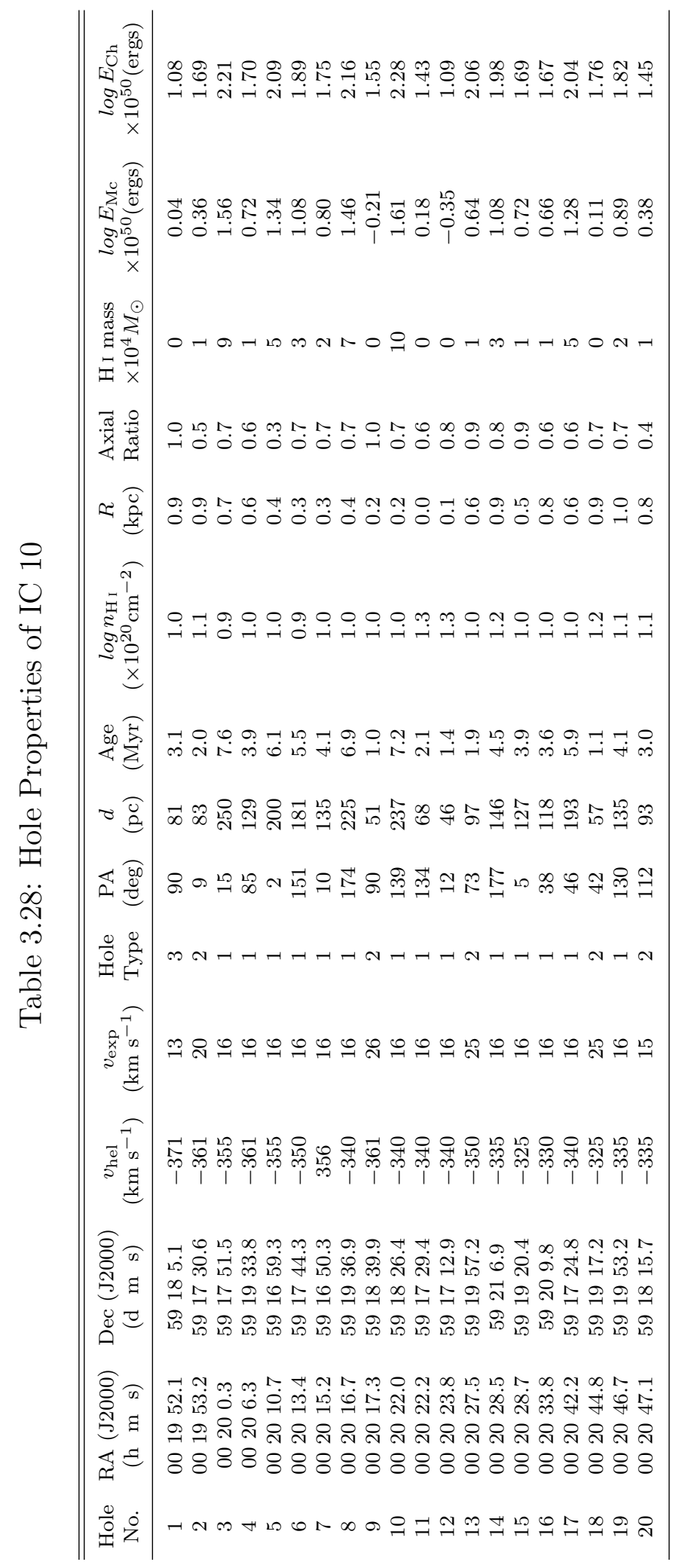




\subsubsection{IC 1613}

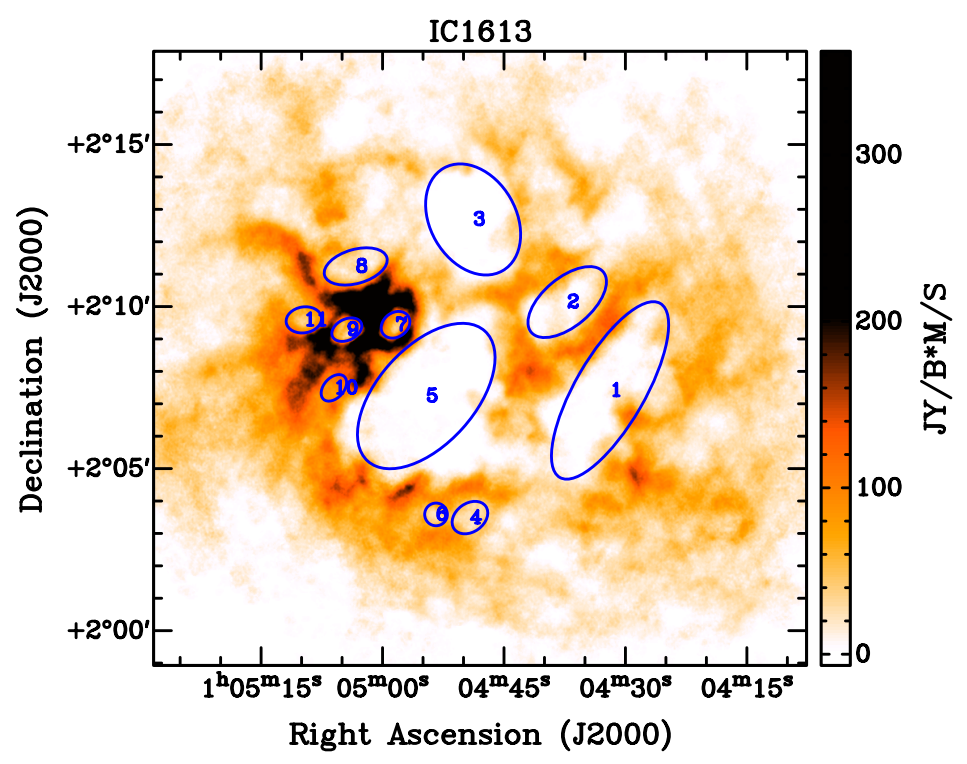

Figure 3.28: H I holes in the ISM of IC 1613 (marked with ellipses). Numbers inside the ellipse indicate the hole number from the catalog. Colors show the types of the holes: Blue ellipses are for Type 1, black are for Type 2, and red are for Type 3 holes.

IC 1613 (PGC 3844, UGC 668, DDO 8) is also a Local Group dwarf. It lies in the constellation of Cetus at a distance of 0.7 kpc. Lozinskaya et al. in 2003 [100] found three large H I shells with expansion velocities of 15 to $18 \mathrm{~km} \mathrm{~s}^{-1}$. Silich et al. [101] recalculated the expansion velocities for those regions to be 10 to $20 \mathrm{~km} \mathrm{~s}^{-1}$. In our data, since all 11 holes we detected were Type 1, we used the velocity dispersion (6 $\mathrm{km} \mathrm{s}^{-1}$ ) of the quiescent regions of the galaxy as the expansion rate of the holes. The estimated scale height of this galaxy is $223 \mathrm{pc}$. 


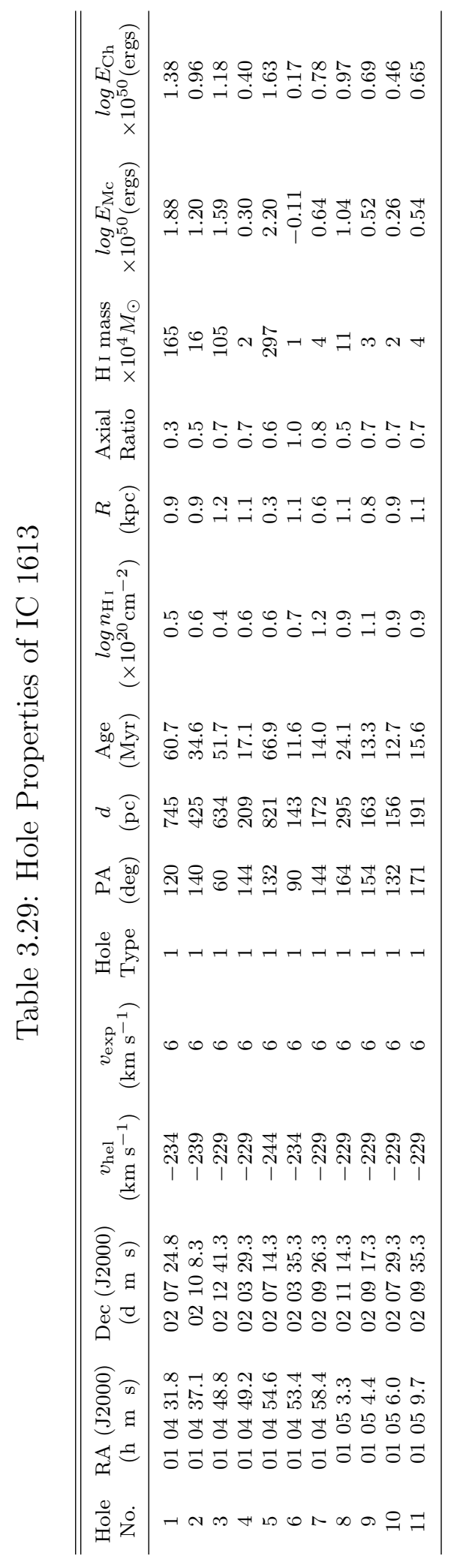




\subsubsection{LGS 3}

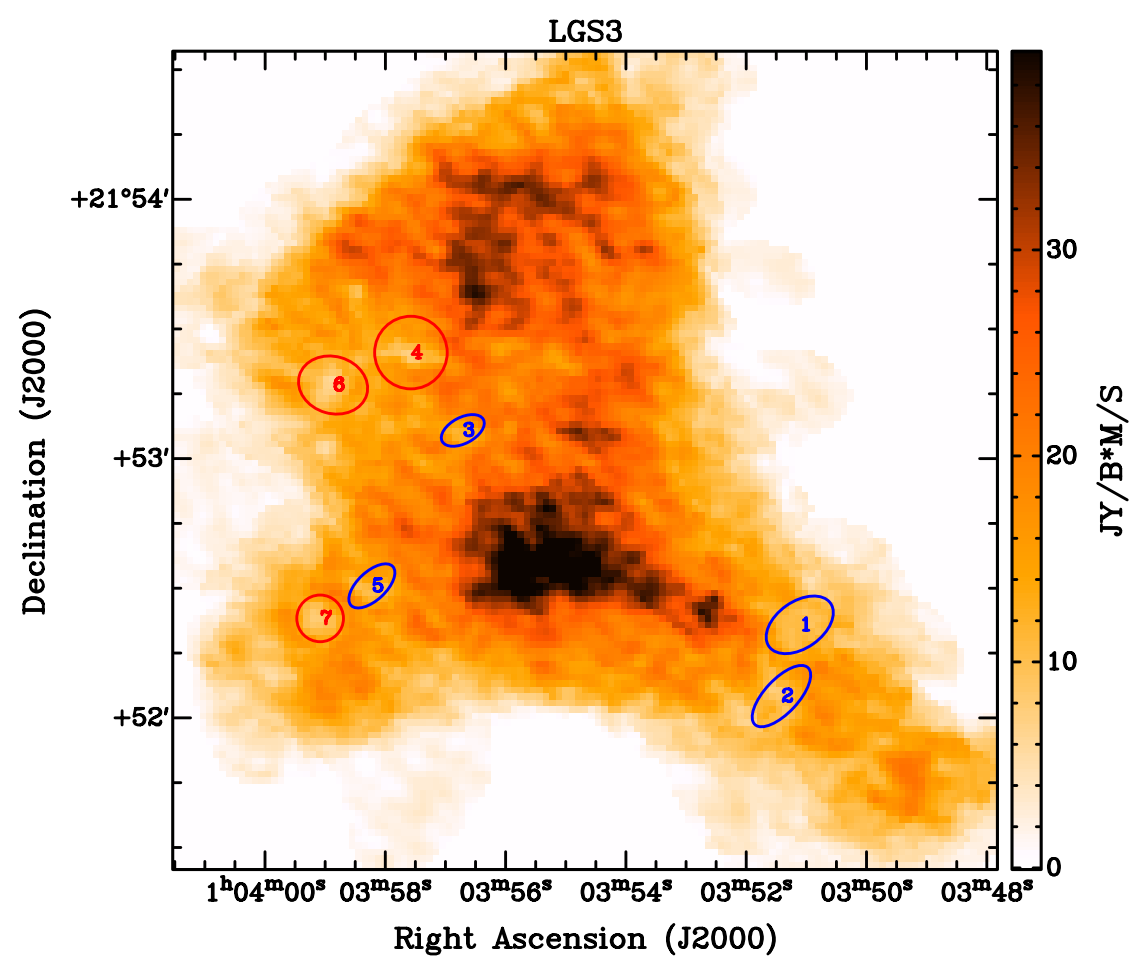

Figure 3.29: H I holes in the ISM of LGS 3 (marked with ellipses). Numbers inside the ellipse indicate the hole number from the catalog. Colors show the types of the holes: Blue ellipses are for Type 1, and red are for Type 3 holes.

LGS 3, also known as as the Pisces Dwarf or PGC 3792, is one of the smallest members of the Local Group, lies about 0.7 Mpc away, and is suspected to be a satellite of the Triangulum galaxy. Among the LITTLE THINGS galaxies, it has the smallest H I disk radius of $(0.4 \mathrm{kpc})$, and smallest scale height of (61 pc). The velocity dispersion of the gas is also the lowest $\left(\approx 5 \mathrm{~km} \mathrm{~s}^{-1}\right)$ in the sample. The galaxy has seven quite small holes including the smallest hole $(27 \mathrm{pc})$ in our catalog. 


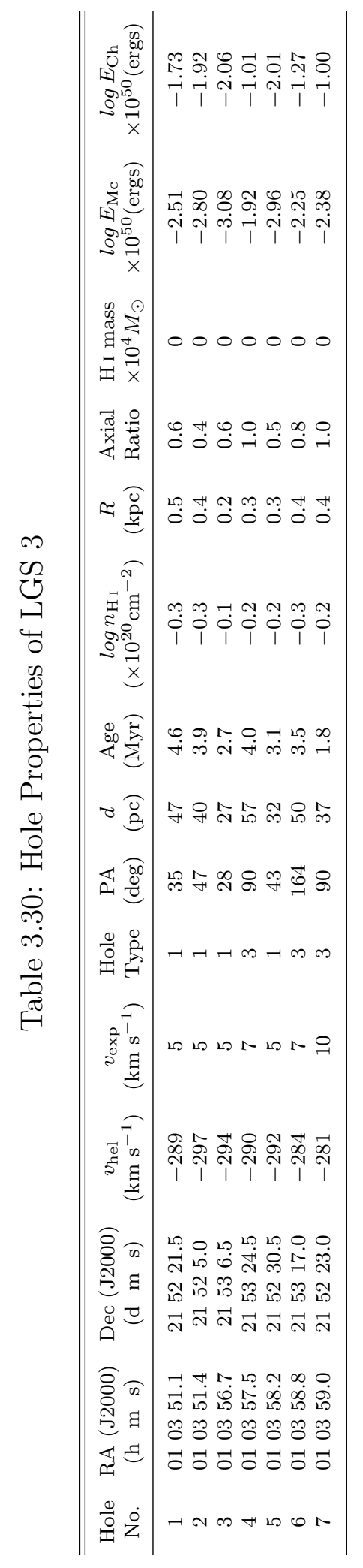




\subsubsection{M81dwA}

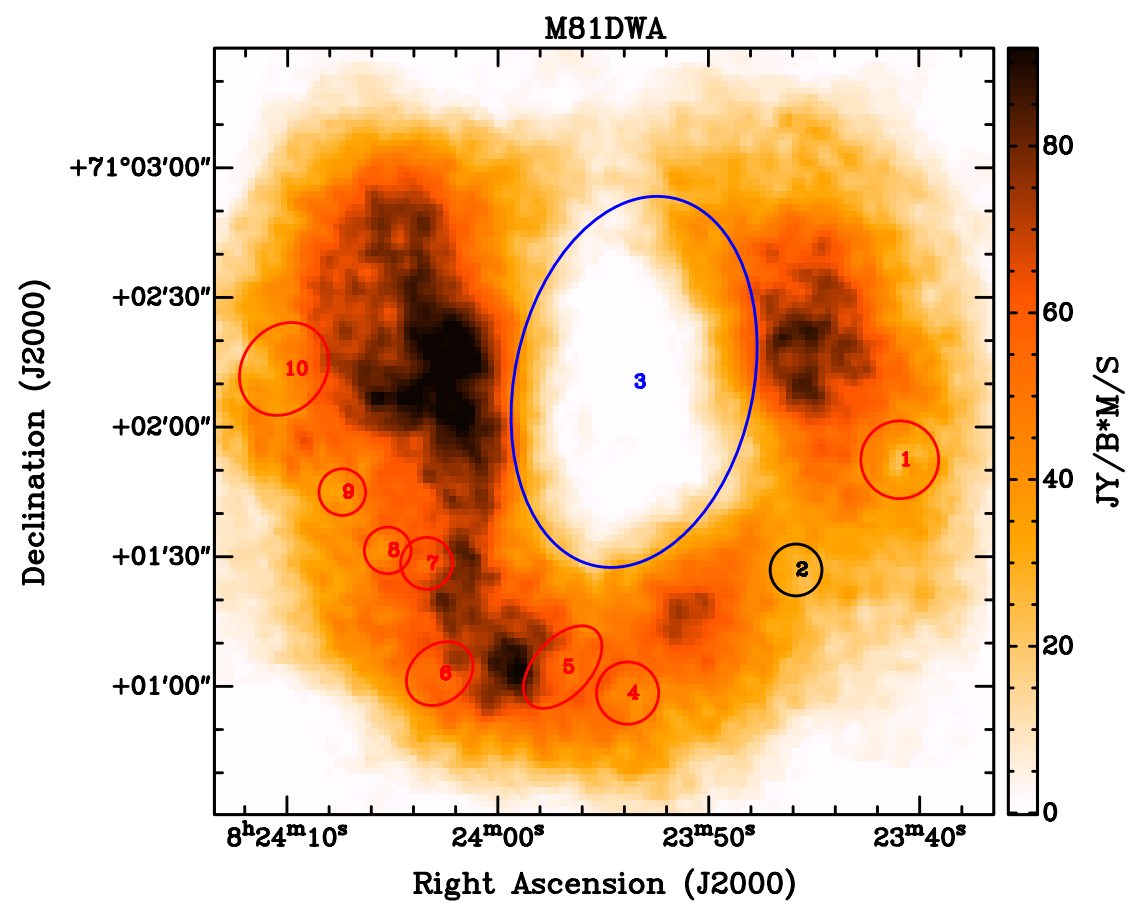

Figure 3.30: H I holes in the ISM of M81dwA (marked with ellipses). Numbers inside the ellipse indicate the hole number from the catalog. Colors show the types of the holes: Blue ellipses are for Type 1, black are for Type 2, and red are for Type 3 holes.

M81dwA (PGC 23521) is one of the extremely faint (V-band magnitude $\approx-11.7$ ) dwarfs in the M81 group, and is at a distance of 3.5 Myr. The inner part of the H I disk of the galaxy is almost covered by a giant kiloparsec-sized Type 1 hole. The galaxy also contains one Type 2 and eight Type 3 holes in the outer part of the disk. 


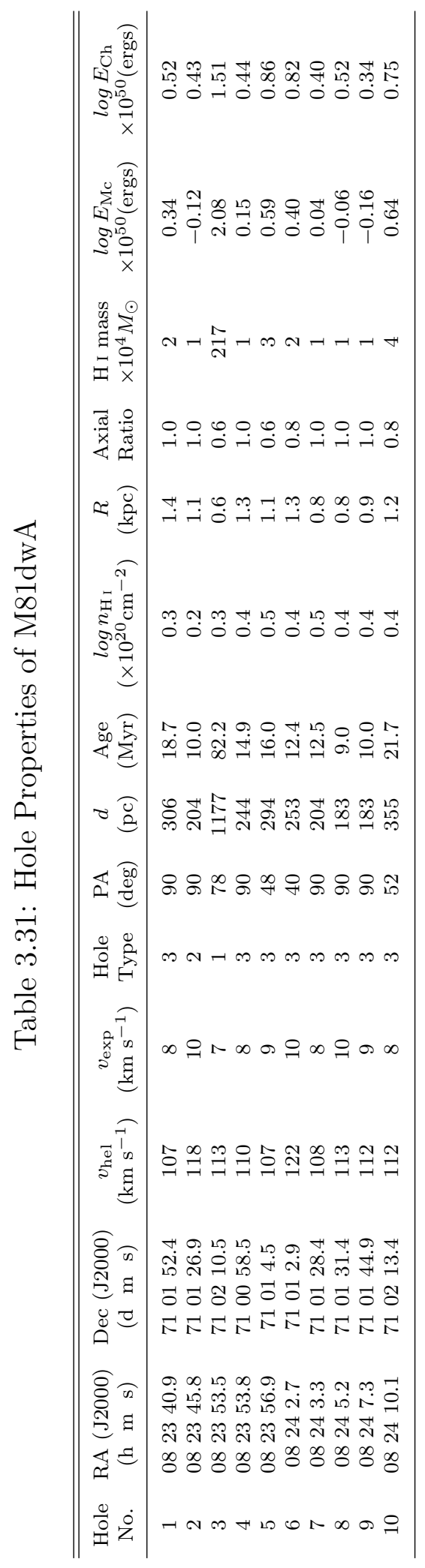




\subsubsection{Mrk 178}

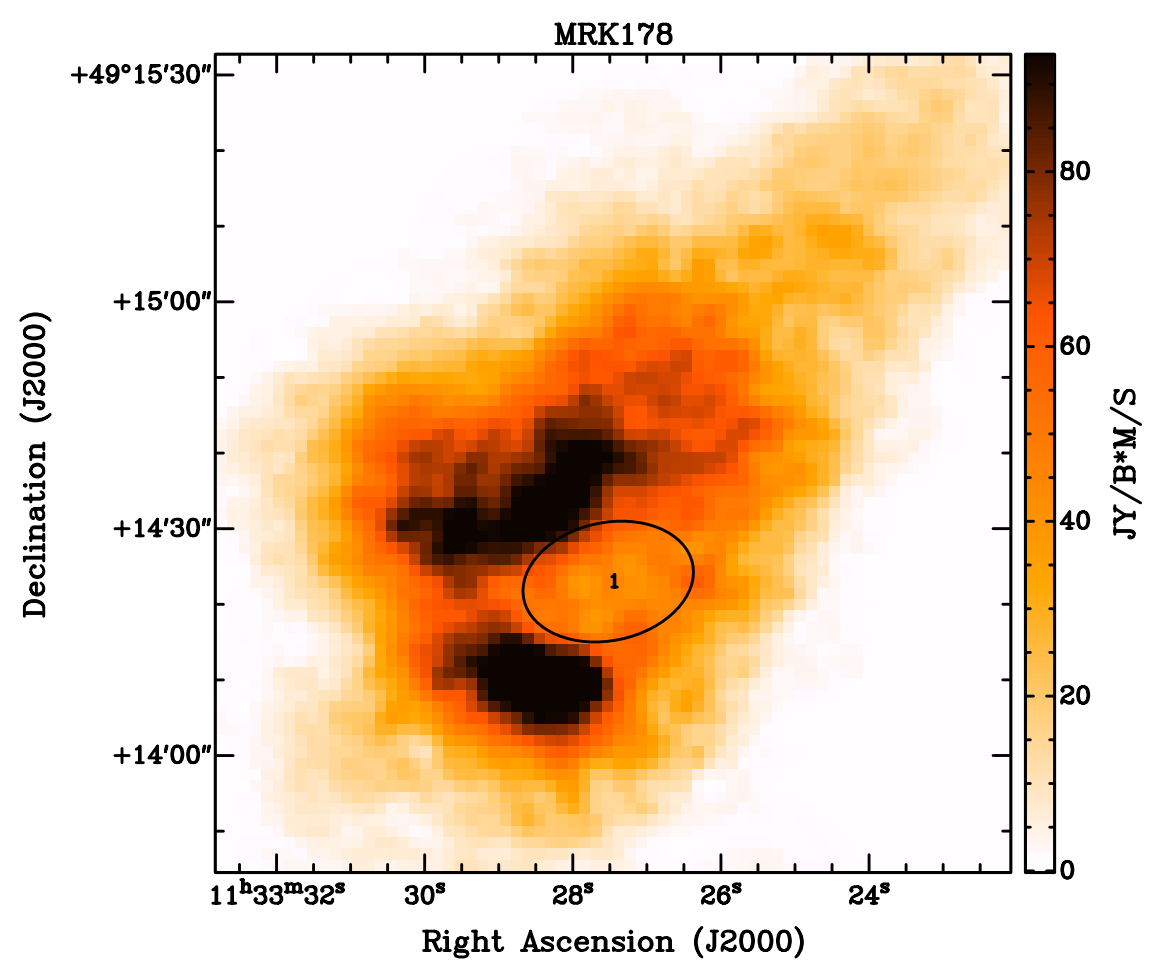

Figure 3.31: H I hole in the ISM of Mrk 178 (marked with ellipse). Number inside the ellipse indicates the hole number from the catalog. Black ellipse is for Type 2 hole.

Mrk 178 (UGC 6541, PGC 35684) is a BCD located $3.9 \mathrm{kpc}$ away from the Milky Way in the Canes Venatici I Group, and has a V-band magnitude of -14.1. The H I structure of the galaxy was studied by Stil and Israel in 2002 [102] but they didn't report on any notable H I structures. We detected a large Type 2 hole expanding with $\approx 16 \mathrm{~km} \mathrm{~s}^{-1}$ in the inner part of the disk. 


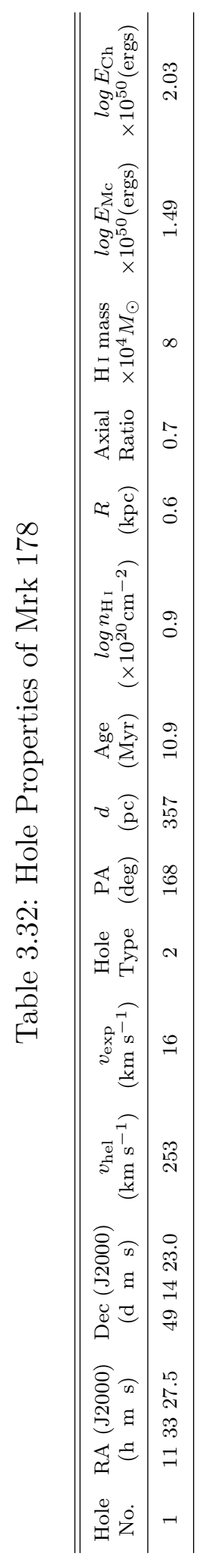




\subsubsection{NGC 1569}

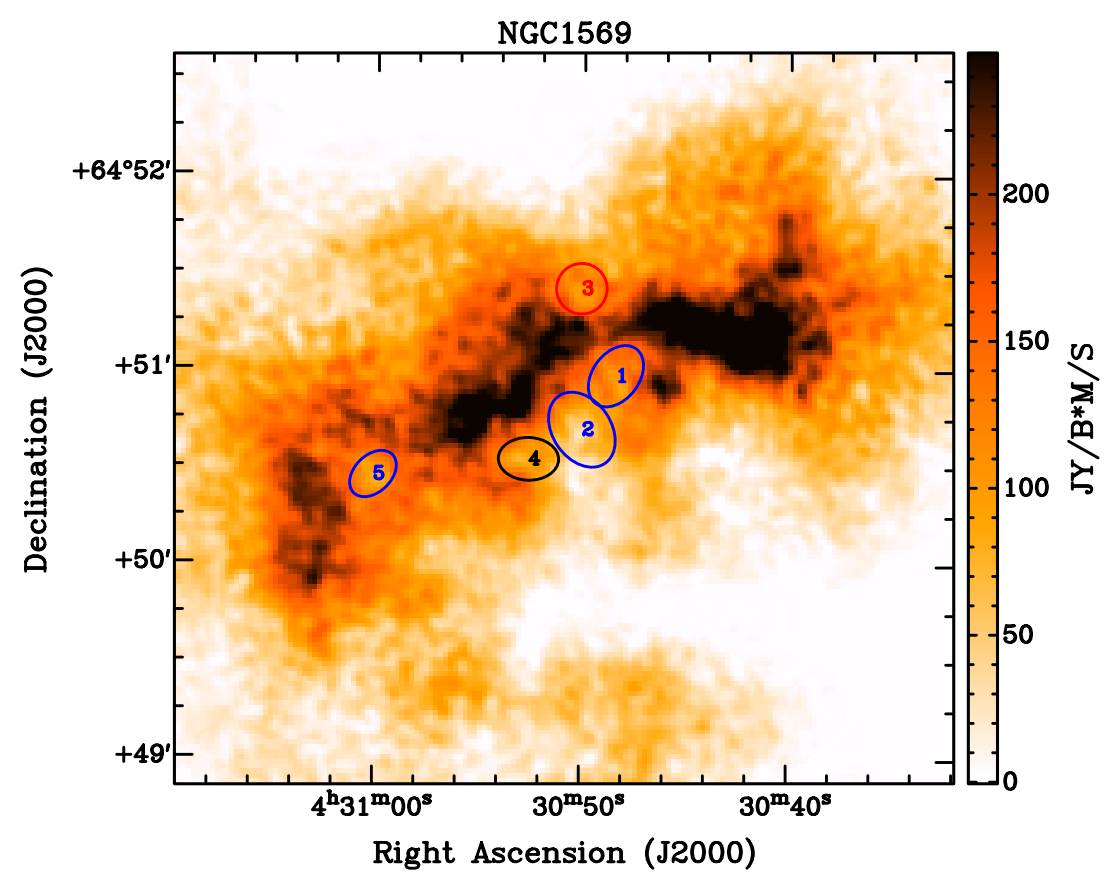

Figure 3.32: H I holes in the ISM of NGC 1569 (marked with ellipses). Numbers inside the ellipse indicate the hole number from the catalog. Colors show the types of the holes: Blue ellipses are for Type 1, black are for Type 2, and red are for Type 3 holes.

NGC 1569 (UGC 3056, VIIZw 16 or Arp 210) has less total H I gas in comparison to other dwarfs, has recently experienced a starburst, and has hot gas outflows. Therefore, it might be transitioning from a dwarf irregular to a dwarf elliptical galaxy [86, 103-105]. The galaxy is about $3.4 \mathrm{Mpc}$ away in the IC 342 (Maffei 1) Group and in the constellation Camelopardalis. We estimated the scale height of the galaxy to be $\approx 219$ pc. The galaxy has five holes, with the highest expansion velocities in our sample, ranging from 22 to $30 \mathrm{~km} \mathrm{~s}^{-1}$. 


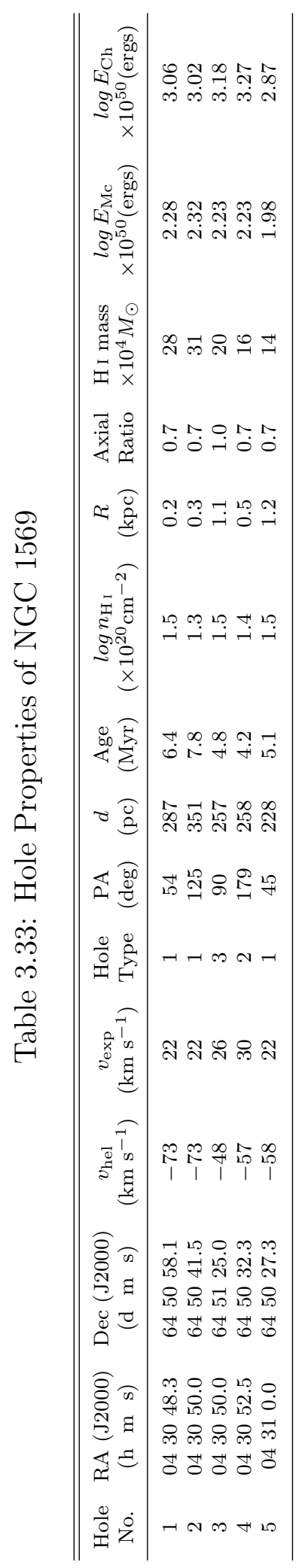




\subsubsection{NGC 2366}

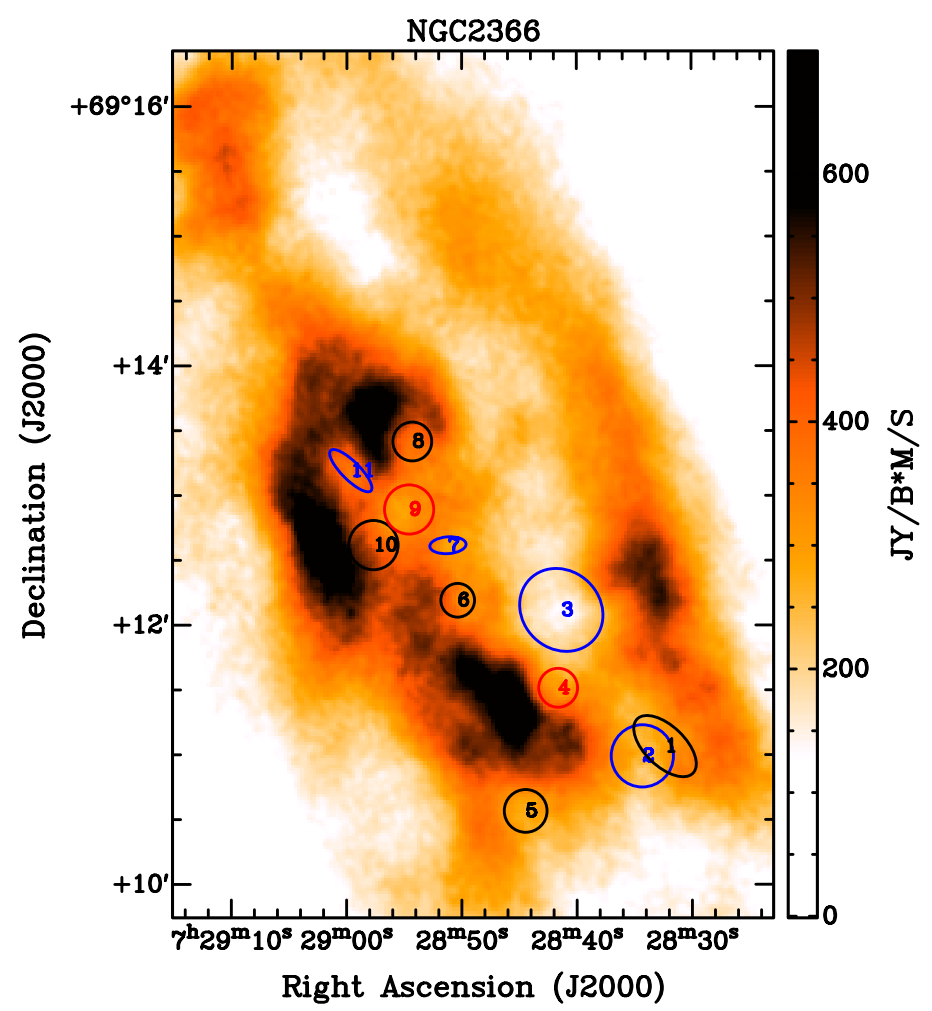

Figure 3.33: H I holes in the ISM of NGC 2366 (marked with ellipses). Numbers inside the ellipse indicate the hole number from the catalog. Colors show the types of the holes: Blue ellipses are for Type 1, black are for Type 2, and red are for Type 3 holes.

NGC 2366, PGC 21102, UGC 3851, or DDO 42 is an M81 Group member located in the constellation Camelopardalis, and is at a distance of $3.4 \mathrm{Mpc}$. We detected 11 H I holes in the ISM of the galaxy. In the northern part of the H I disk, there appears to be a large Type 1 hole, but it is just a region where the flux density is below the threshold. The estimated scale height of the galaxy is about $400 \mathrm{pc}$. 


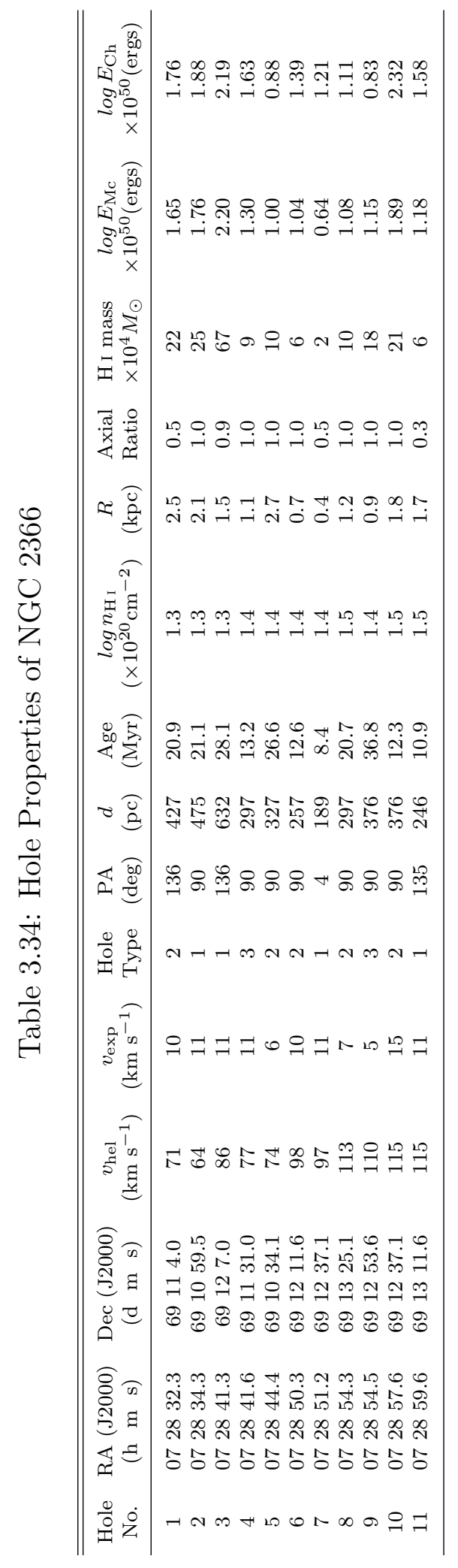




\subsubsection{NGC 3738}

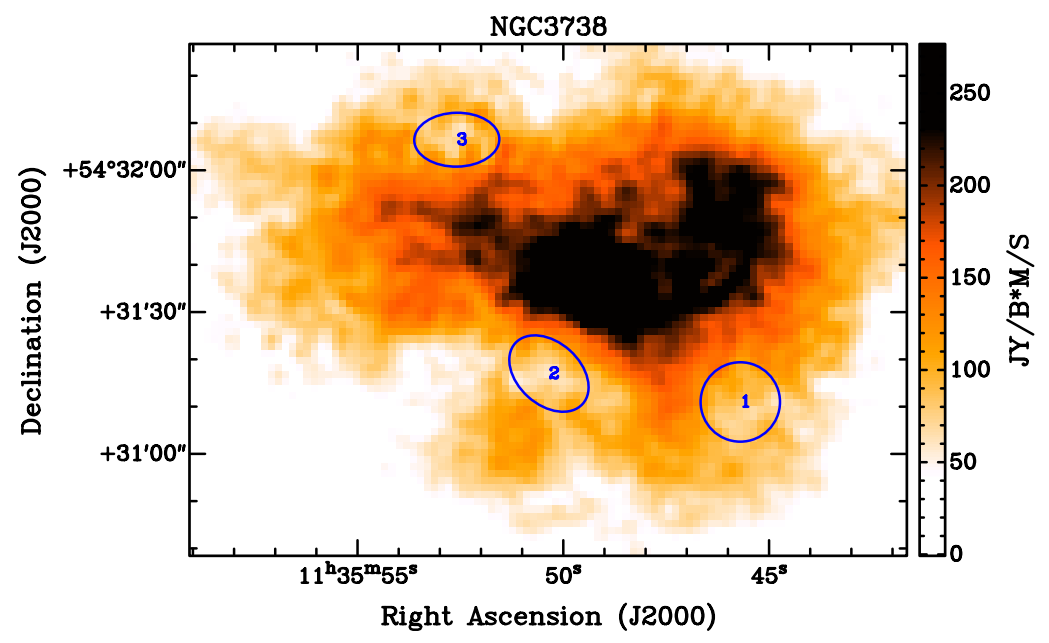

Figure 3.34: H I holes in the ISM of NGC 3738 (marked with ellipses). Numbers inside the ellipse indicate the hole number from the catalog. Colors show the types of the holes: Blue ellipses are for Type 1 holes.

Because NGC 3738 (UGC 6565, Arp 234) is oriented face-on to us $\left(i=0^{\circ}\right.$ ) [84], it is not possible to estimate a dynamical H I mass and scale height for the galaxy. The galaxy is located at a distance of $1 \mathrm{Mpc}$ in the Canes Venatici I Group and has a V-band magnitude of -17.1 . It contains five small completely broken holes. 


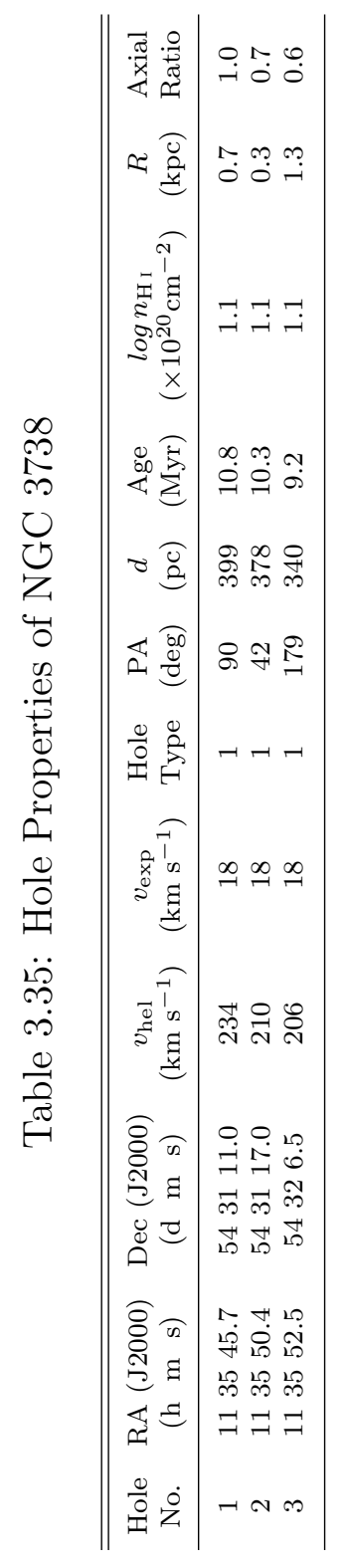




\subsubsection{NGC 4163}

NGC 4163, also known as UGC 7199 and PGC 38881, is located at a distance of about $2.9 \mathrm{Mpc}$. According to our selection criteria, there are no holes in this galaxy. We estimated the scale height of the galaxy to be about $139 \mathrm{pc}$ and the $\mathrm{H}$ I disk length as 800 pc. 


\subsubsection{NGC 4214}

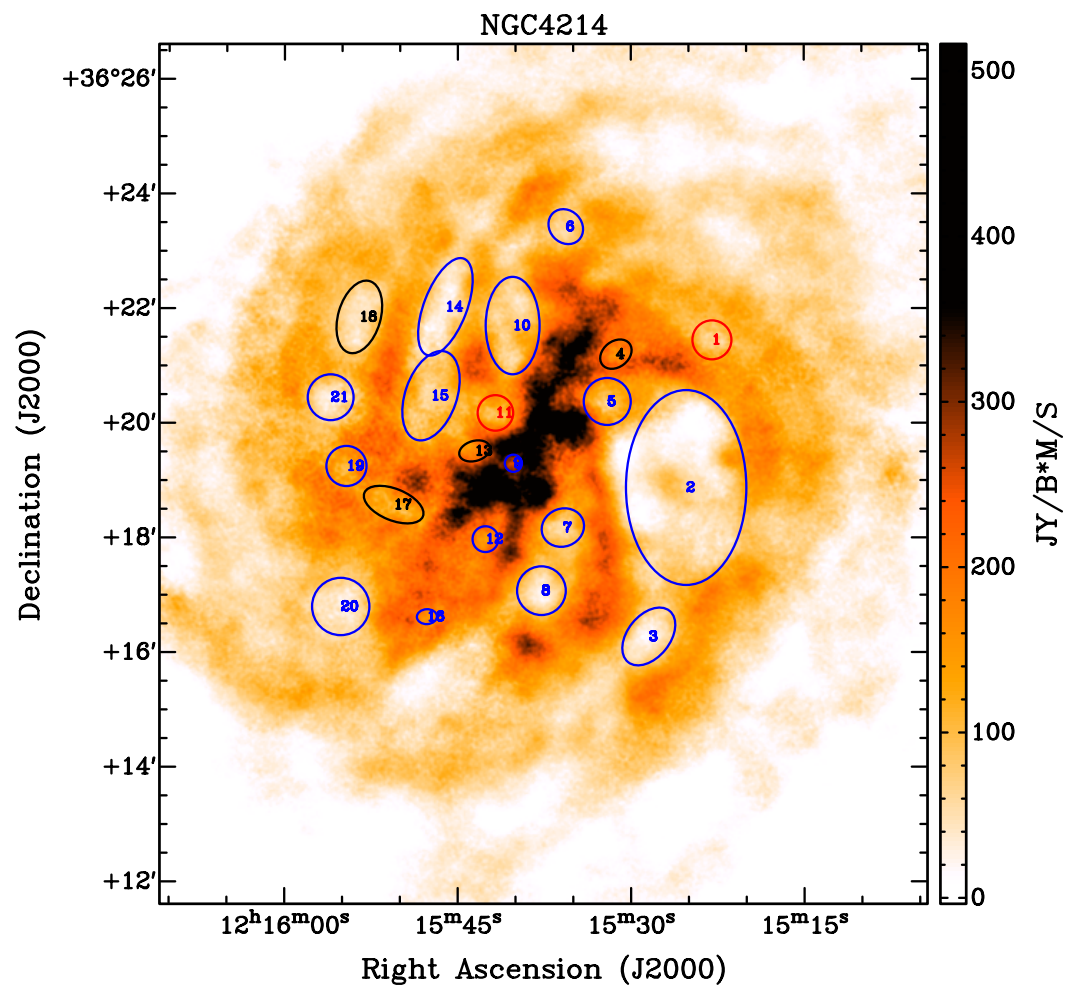

Figure 3.35: H I holes in the ISM of NGC 4214 (marked with ellipses). Numbers inside the ellipse indicate the hole number from the catalog. Colors show the types of the holes: Blue ellipses are for Type 1, black are for Type 2, and red are for Type 3 holes.

NGC 4214 (UGC 7278, PGC 39225) is a Magellanic starburst dwarf located at a distance of $3 \mathrm{Mpc}$ [106] in the M94 Group. We credited only 21 holes out of 55 initially detected structures, which is less than half of the 56 holes found by Bagetakos et al. [33]. Among them about $71 \%$ are Type 1 holes. The galaxy also contains four holes larger than a kiloparsec, including the largest hole $(\approx 2.3 \mathrm{kpc})$ detected in the LITTLE THINGS sample. The galaxy also has the highest rotational velocity (77 $\mathrm{km} \mathrm{s}^{-1}$ ) among all. The estimated $\mathrm{H}$ I scale height and $\mathrm{H}$ I disk radius of the galaxy are about $301 \mathrm{pc}$ and $6.3 \mathrm{kpc}$ respectively. 


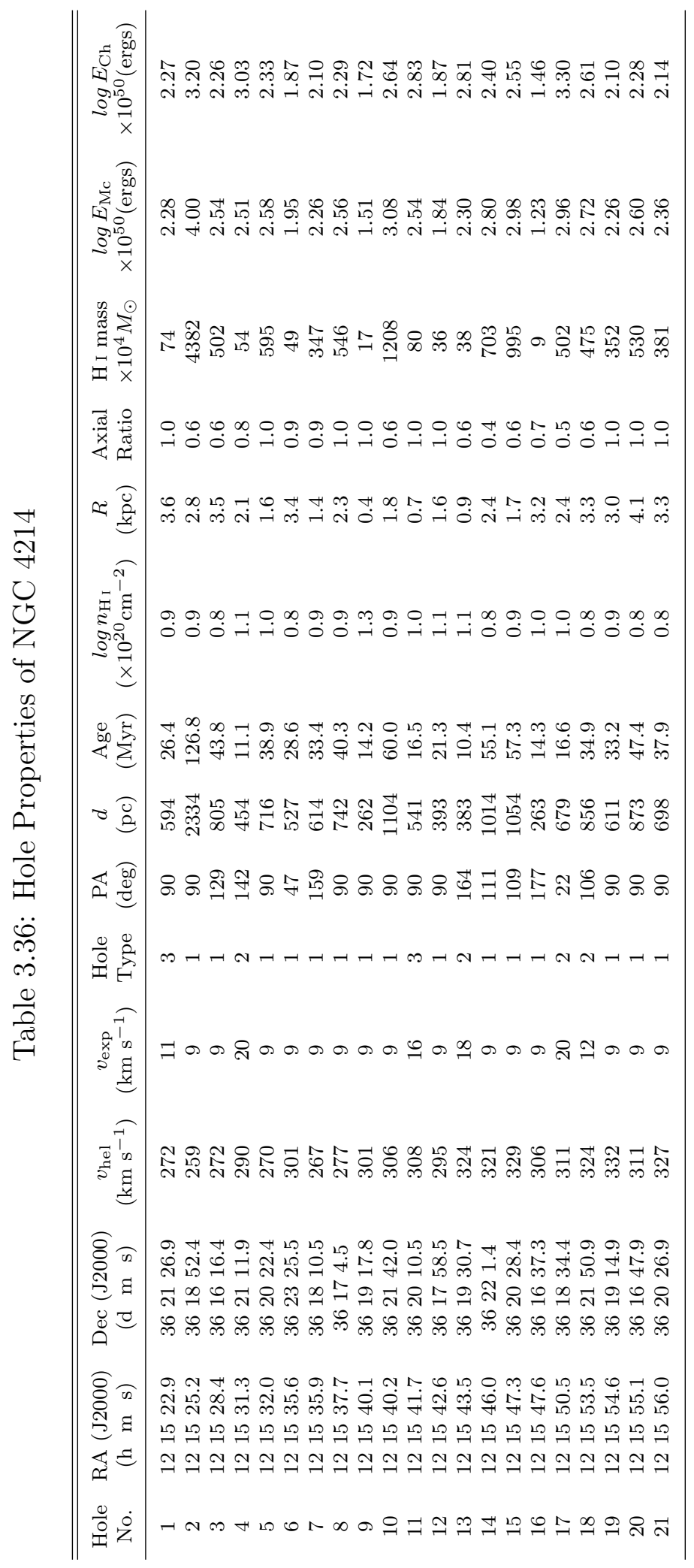




\subsubsection{SagDIG}

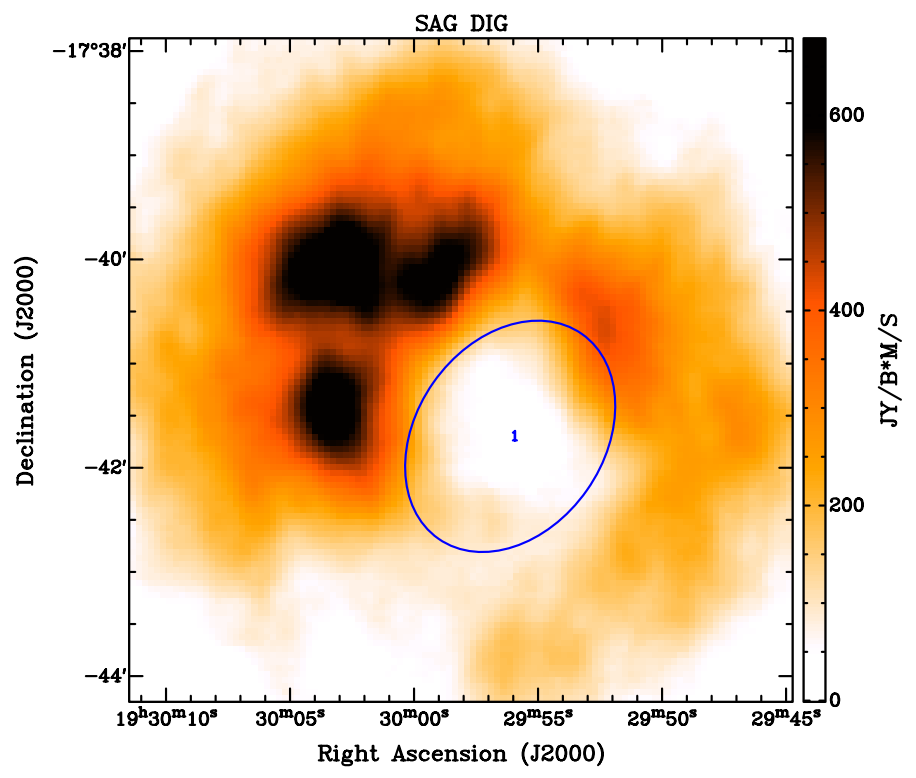

Figure 3.36: H I hole in the ISM of SagDIG (marked with ellipse). Number inside the ellipse indicates the hole number from the catalog. Blue ellipse is for Type 1 hole.

The Sagittarius dwarf irregular galaxy (SagDIG), also known as Lowal's Object or PGC 63287, is an isolated satellite of the Milky Way about 1.1 Mpc away. The H I disk of SagDIG is dominated by a large Type 1 Hole of diameter $\approx 666 \mathrm{pc}$. The scale height of the galaxy is about $653 \mathrm{pc}$ which is the highest in LITTLE THINGS sample according to our estimation. 


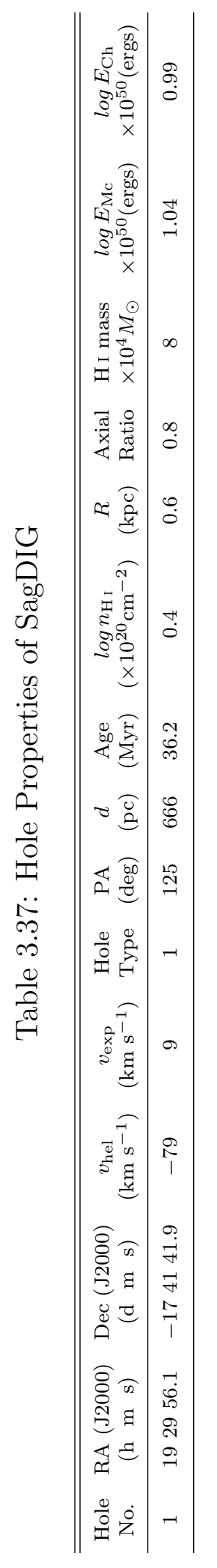




\subsubsection{UGC 8508}

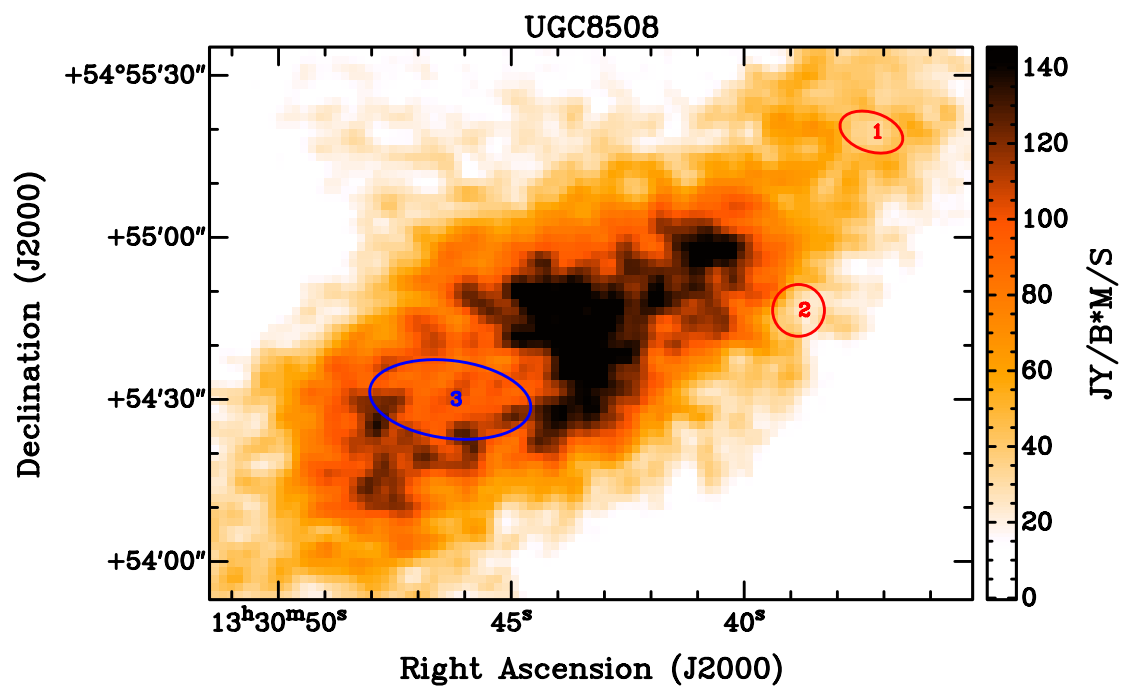

Figure 3.37: H I holes in the ISM of UGC 8508 (marked with ellipses). Numbers inside the ellipse indicate the hole number from the catalog. Colors show the types of the holes: Blue ellipses are for Type 1, and red are for Type 3 holes.

UGC 8508 (PGC 47495, IZw 60) is 2.6 Mpc away from the Milky Way in the M101 Group with magnitude -13.6. The galaxy has a large Type 1 hole near its center and two intact holes are in the periphery of the H I disk. The estimated H I scale height and the $\mathrm{H}$ I disk radius of the galaxy are $\approx 144 \mathrm{pc}$ and $\approx 1.4 \mathrm{kpc}$ respectively. 


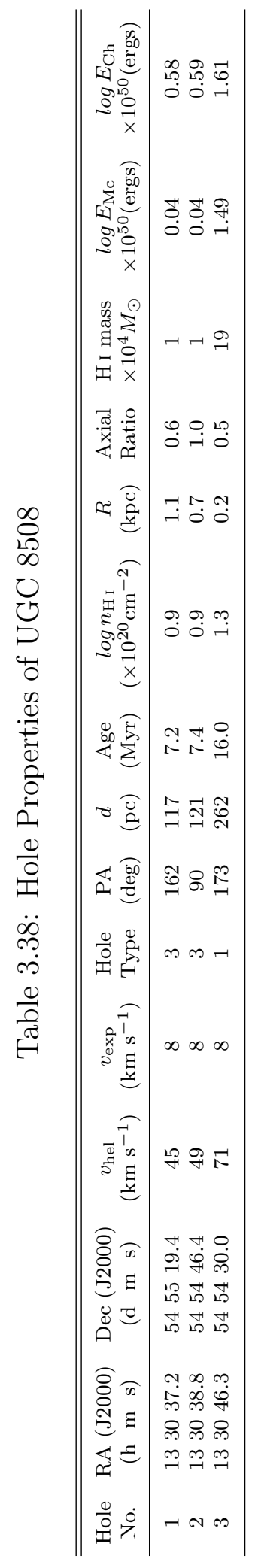




\subsubsection{WLM}

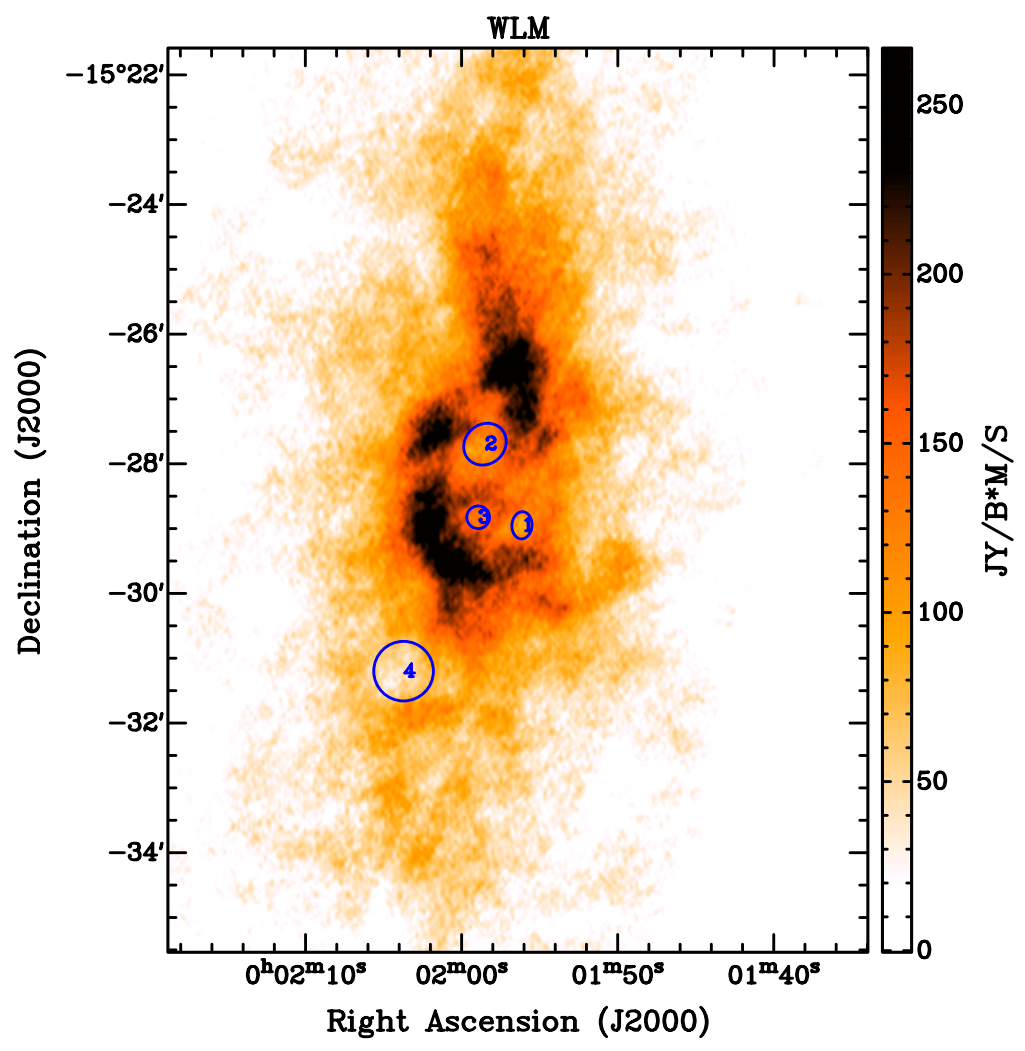

Figure 3.38: H I holes in the ISM of WLM (marked with ellipses). Numbers inside the ellipse indicate the hole number from the catalog. Blue ellipses are for Type 1 holes.

Wolf-Lundmark-Melott (WLM), also known as UGCA 444 or DDO 221, is an isolated dwarf which lies at the outer edges of the Local Group. It is located about a distance of $1 \mathrm{Mpc}$ from the Milky Way with a V-band magnitude of -14.4. All four holes of this galaxy are Type 1 . The absence of Type 2 and Type 3 holes might be due to its small H I scale height, which is only about $96 \mathrm{pc}$. 


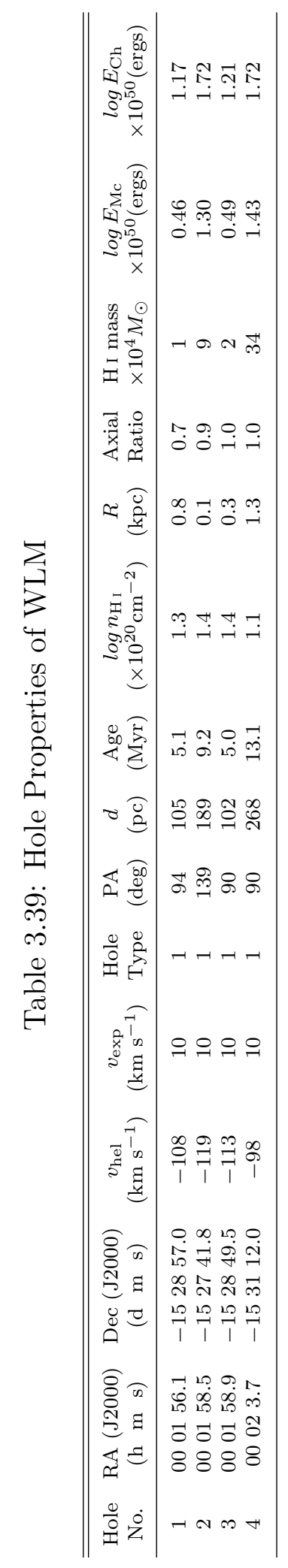




\subsubsection{VIIZw403}

VIIZw 403, also known as UGC 6456 and PGC 5286, is an isolated dwarf at a distance

of about 4.4 Mpc, and is considered as a member of the M81 Group. Simpson et al. [107] found an H I cavity coincident with the center of the H I velocity field but our criteria didn't define that cavity as a hole. We didn't detect any holes in this galaxy according to our selection criteria.

\subsection{Analysis of the Properties}

Figure 3.39 shows the histogram of the radial distribution of the H I holes in each galaxy as a function of relative frequency in percentage. Only a few galaxies, DDO 43, DDO 46, DDO 47, DDO 50, DDO 53, DDO 70, DDO 87, WLM, NGC 3738 and IC 10 have holes at all radial distances. DDO 52, DDO 75, DDO126, DDO 133, DDO 187, F564-V3 and LGS 3 have more holes in their outskirts, while DDO 63, DDO154, NGC 2366, NGC 4214 and IC 1613 have more holes in their inner regions. In Figure 3.40, a histogram of the radial distribution of all the $\mathrm{H}$ I holes is plotted. About $85 \%$ of the holes are found within $\approx 15 \%$ to $80 \%$ radial distance from the center of the galaxies. 


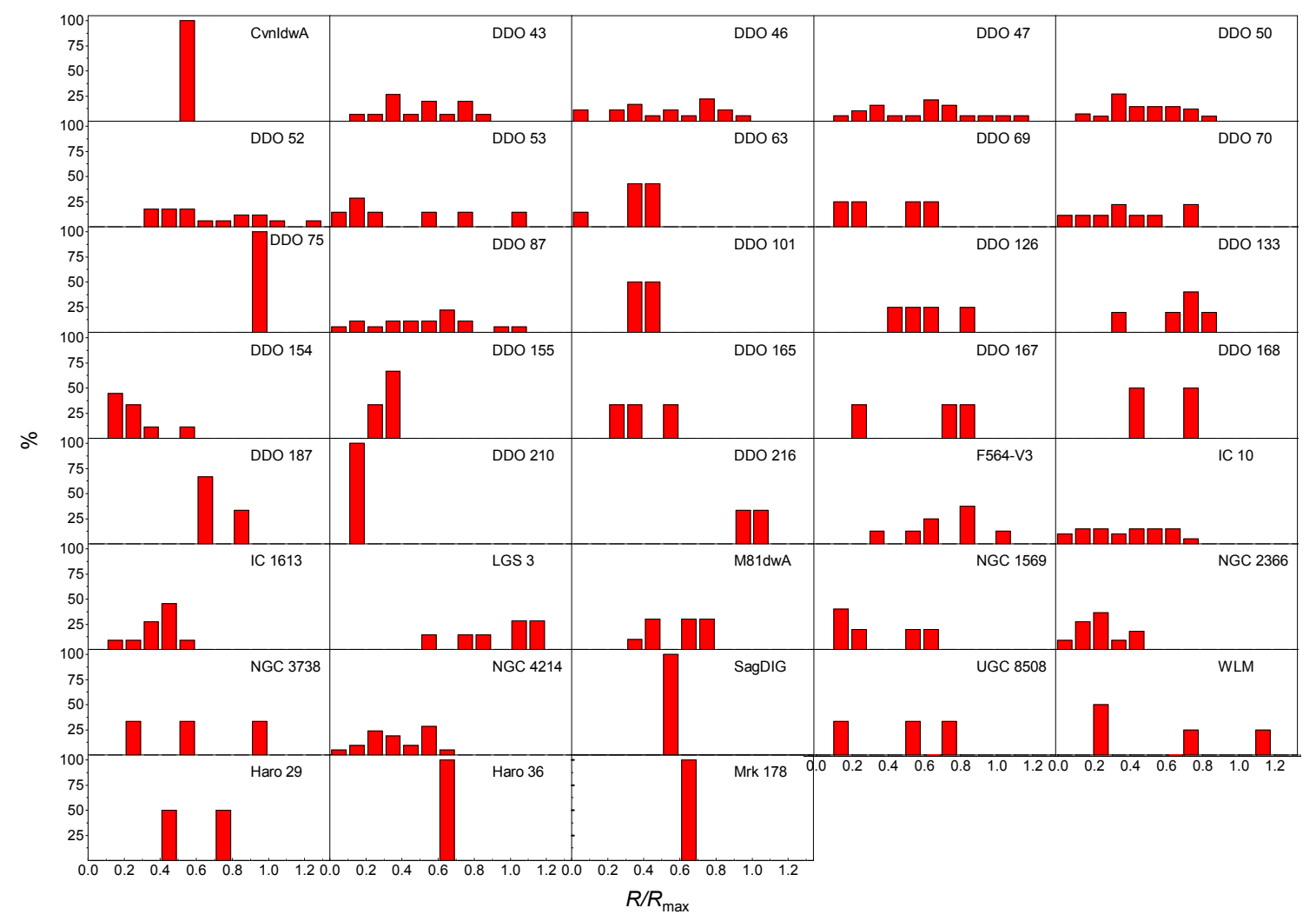

Figure 3.39: Relative radial distribution of $\mathrm{H}$ I holes.

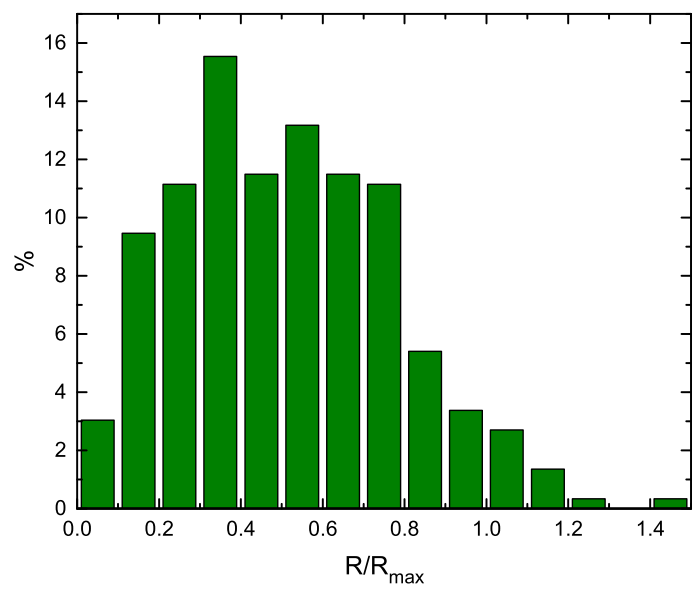

Figure 3.40: Relative radial number distribution of $\mathrm{H}$ I holes for the entire sample. 
The number distribution of the diameter of the holes of each galaxy is shown in Figure 3.41. Each panel shows the histogram of the diameter $(\mathrm{kpc})$ of the holes versus its relative frequency in percentage. DDO 47, DDO 50, DDO 87 and NGC 4214 have a wide range of hole sizes. Figure 3.42, which plots the hole diameters for the entire galaxy sample, shows that approximately $75 \%$ of the holes have diameters less than 500 pc. We have only 22 holes larger than a kiloparsec.

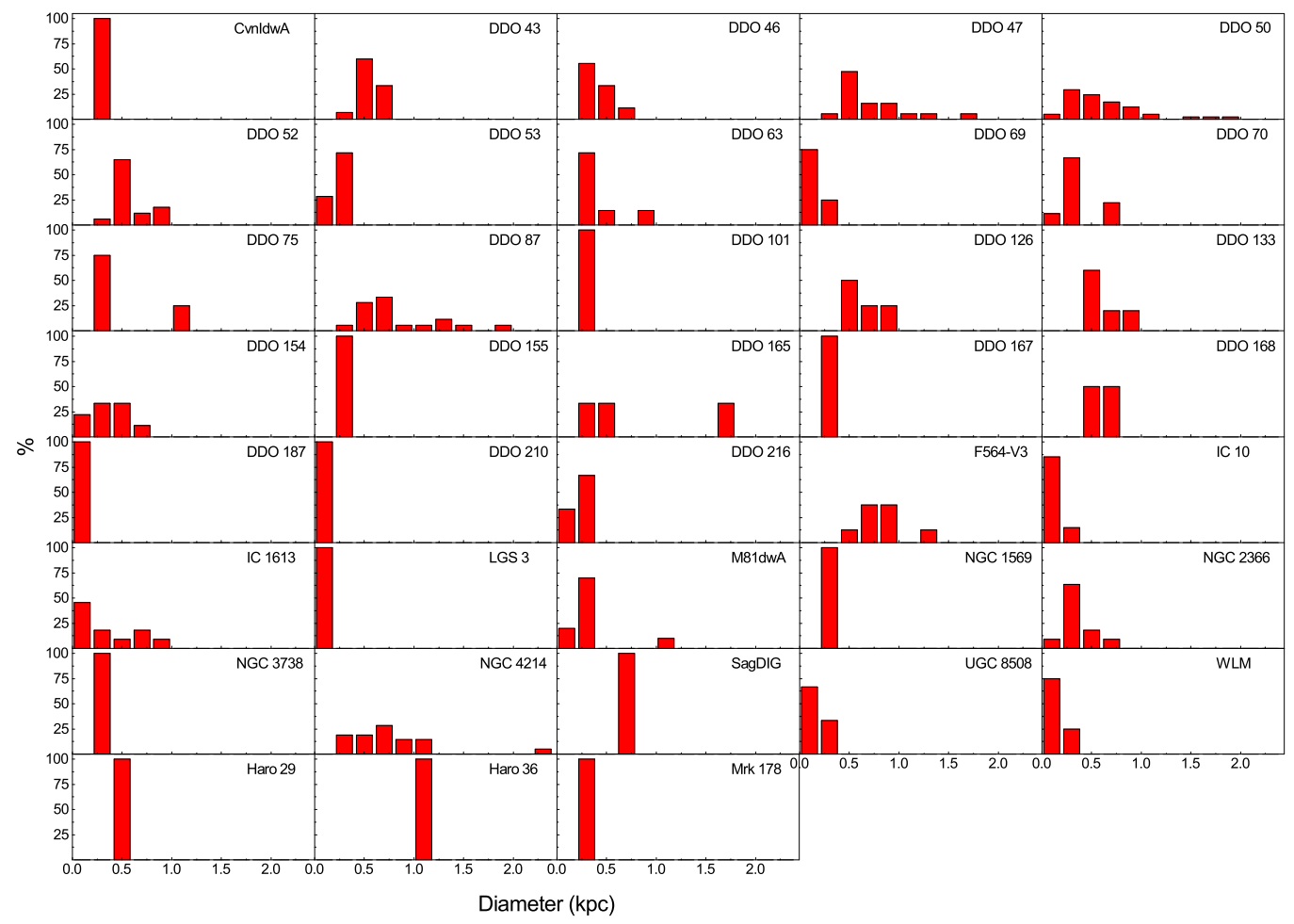

Figure 3.41: Relative number distribution of the size of the $\mathrm{H}$ I holes. 


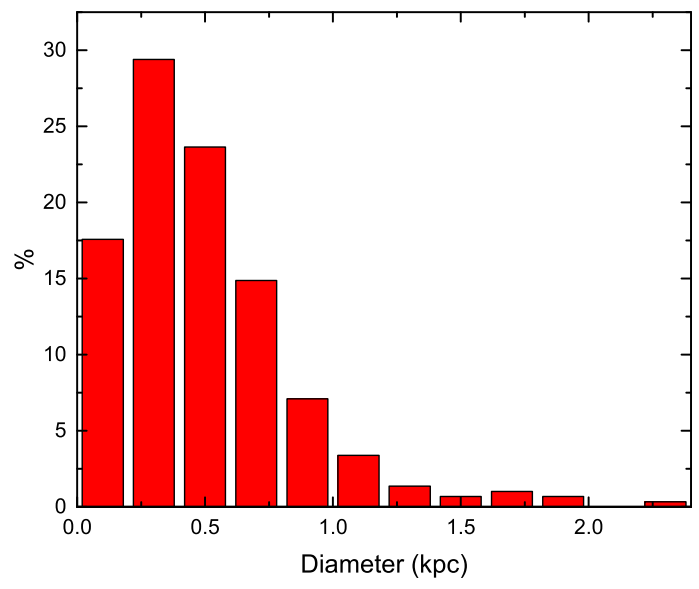

Figure 3.42: Relative number distribution of the size for the $\mathrm{H}$ I holes of entire sample.

Figure 3.43 shows the location and size of all the holes in the sample relative to the normalized V-band scale length of the galaxies. The plot shows that the concentration of the holes is in between one and two V-band scale lengths. 


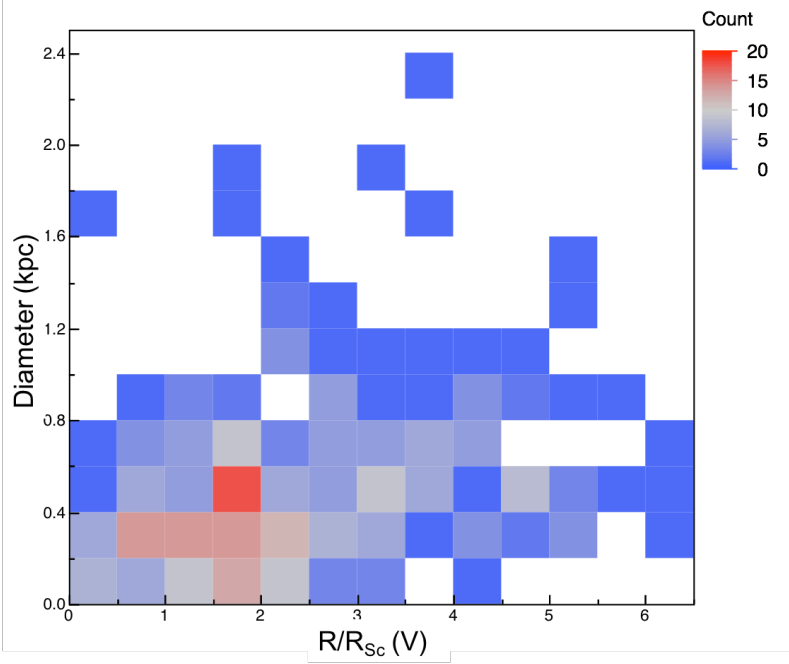

Figure 3.43: Distribution of H I holes: diameter vs. normalized V-band scale length.

The distribution of the expansion velocities of the Type 2 and Type 3 holes is given in Figure 3.44. For some galaxies like DDO 43, DDO 47, DDO 50, DDO 52, DDO 53, F564-V3, LGS 3 and M81dwA, we see a decreasing percentage of holes with higher expansion velocities. Figure 3.45 shows the cumulative distribution of expansion velocities with respect to the location of the holes which shows that most of the holes $(\approx 70 \%)$ are expanding at velocities between 6 to $16 \mathrm{~km} \mathrm{~s}^{-1}$. The plot of the expansion velocities of all the holes against their normalized radial positions is in Figure 3.46. We see that the fastest expanding holes are located more towards the inner/middle parts of the galaxies. 


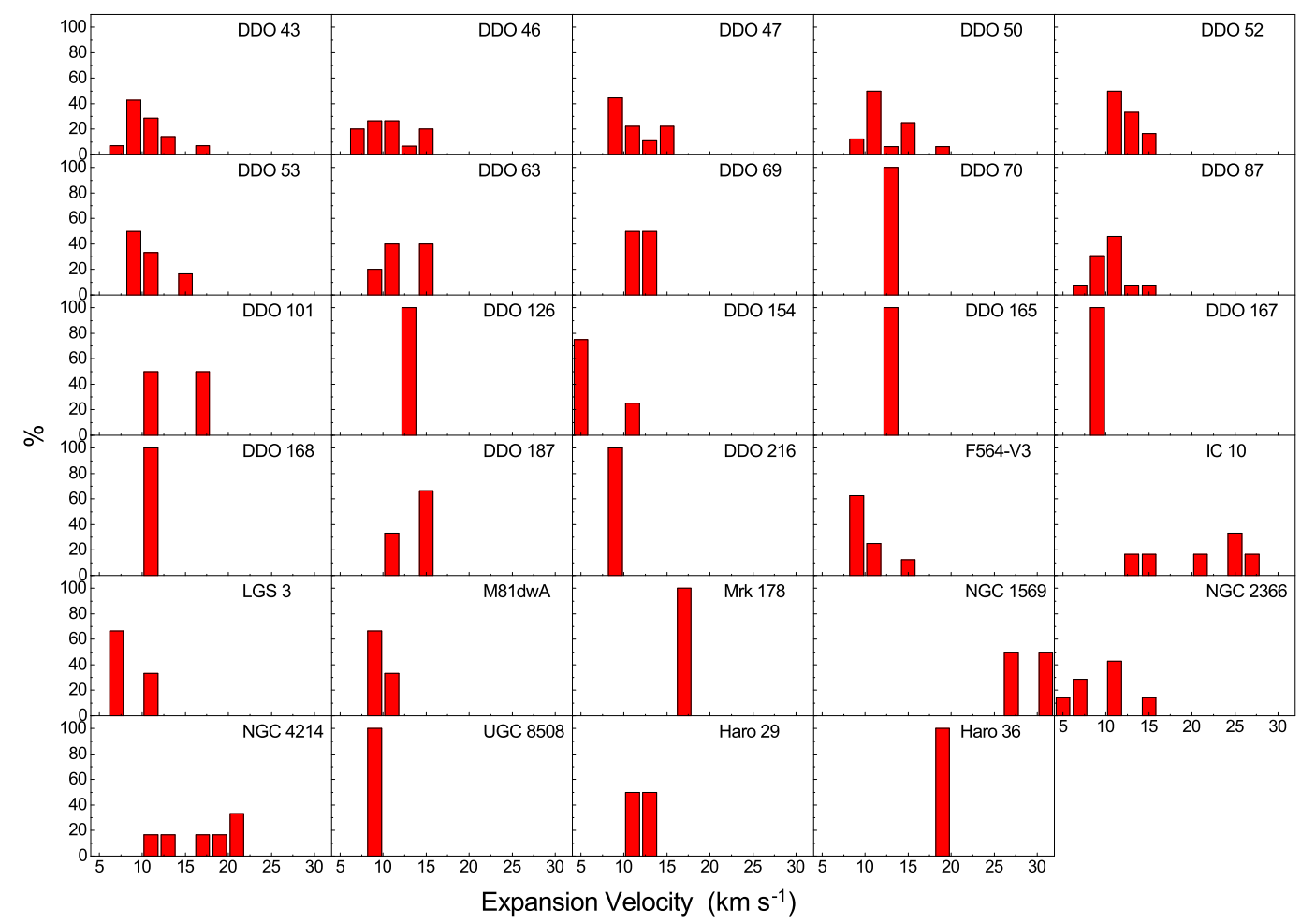

Figure 3.44: Relative number distribution of the expansion velocity of Type 2 and Type 3 holes. 


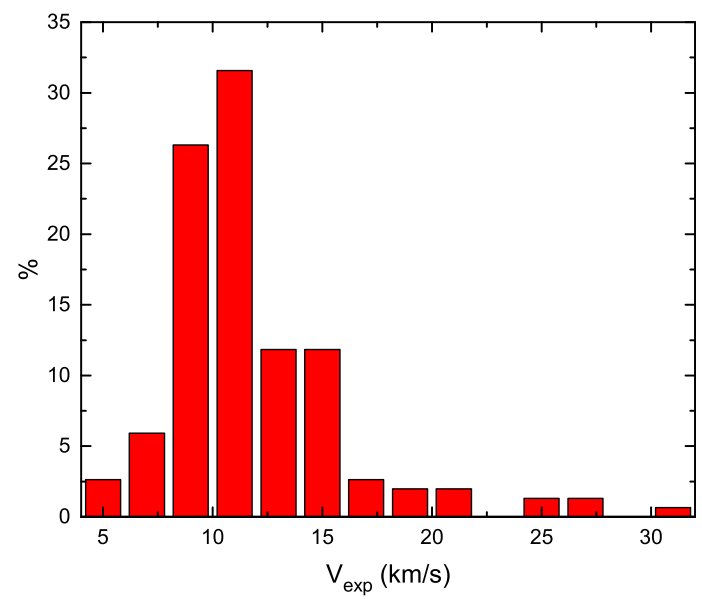

Figure 3.45: Relative number distribution of the expansion velocity of Type 2 and Type 3 holes for the entire sample.

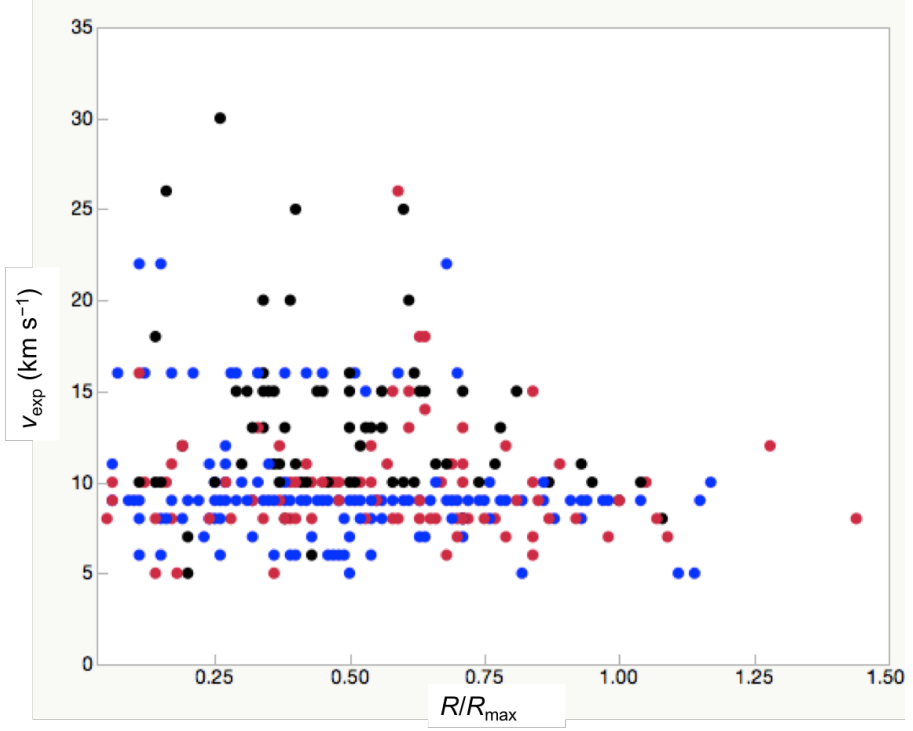

Figure 3.46: Distribution of H I holes: Expansion velocities with respect to the location. Blue colored points are the upper limits of expansion velocities for Type 1 holes, black and red are the expansion velocities of Type 2 and Type 3 holes respectively.

Figure 3.47 shows the histogram of the distribution of the ages of different types of holes. It shows that most of the Type 2 and Type 3 holes have kinetic ages less 
than 40 Myr. Ages for Type 1 holes are estimated as an upper limit because the expansion velocity for Type 1 hole is taken as the velocity dispersion of the quiescent area of that galaxy, which is a lower limit.

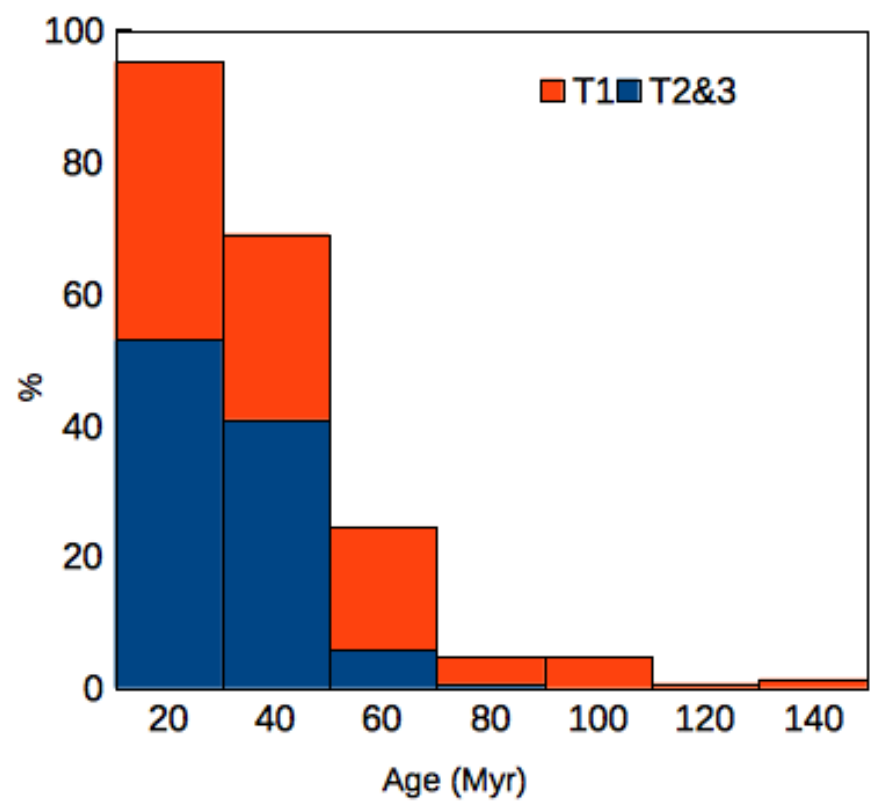

Figure 3.47: Distribution of the kinetic ages of H I holes.

In Figure 3.48, we show the percentage of holes in the inner vs. the outer regions of the galaxies. The dividing line is set by the 'break radius,' which is the distance from the center of the galaxy to the point at which the stellar surface brightness profile changes sharply, indicating a change in the stellar distribution. An example is shown in Figure 3.49 for DDO 75 from Herrmann et al. 2013 [108]; the break radius is at about 1.9 arcmin here. But interestingly, the percentage of holes inside and outside the V-band break radius is nearly constant. 


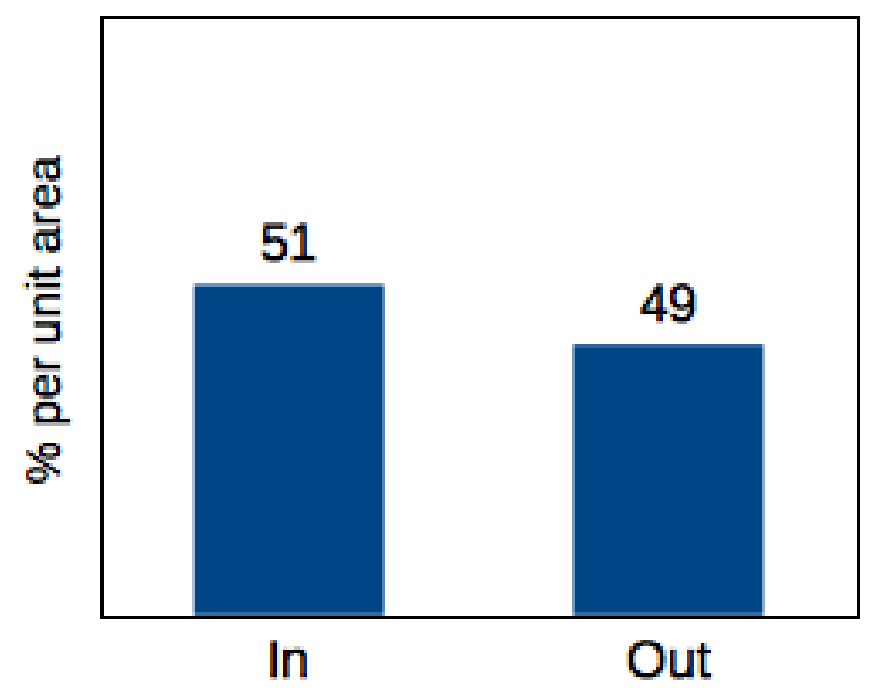

Figure 3.48: Distribution of H I holes per unit area inside and outside of the break radius.
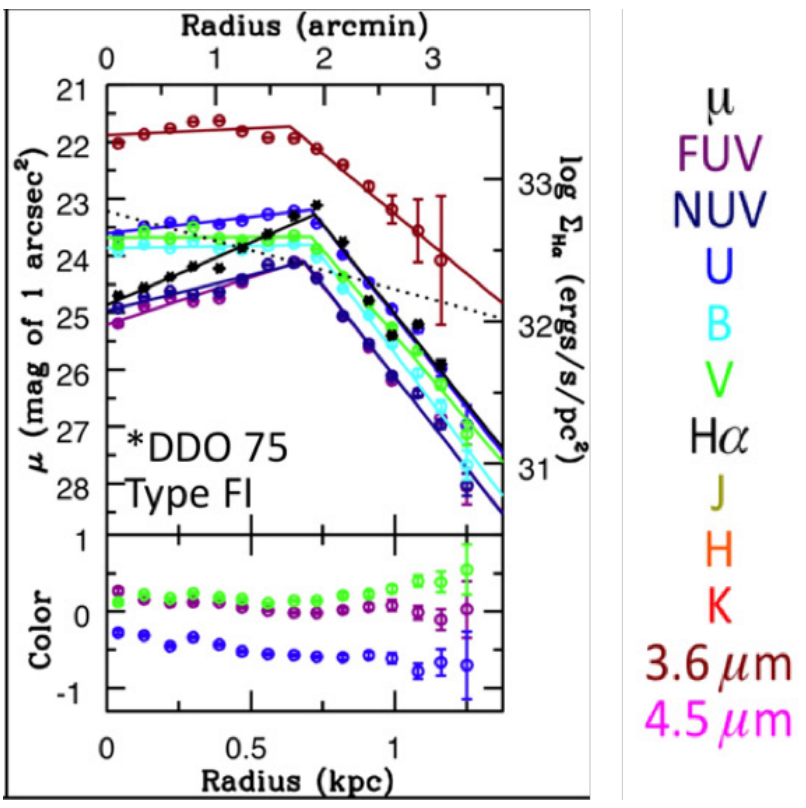

Figure 3.49: Surface brightness profiles and radial color distributions for DDO 75. The dotted black line is the best exponential fit to the V-band data (Herrmann et al. 2013).

The percentage distribution of the logarithm of the energy required to form holes (calculated using McCray and Kafatos method [57]) is given in Figure 3.50. 
From the histograms, we see that there are only a few galaxies having holes which require more than $4 \times 10^{54}$ ergs of energy (in the entire sample, there are 10 holes which require that energy).

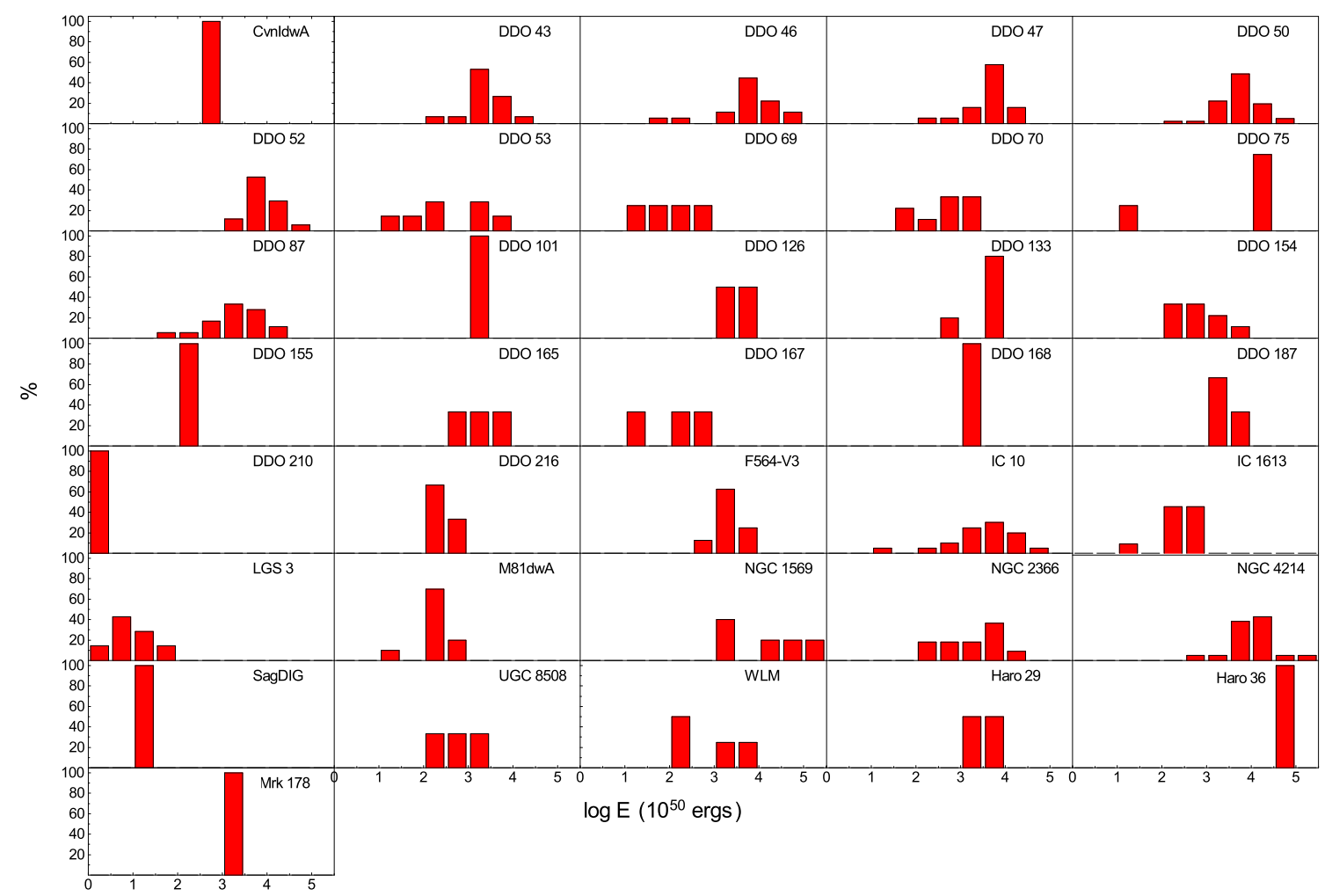

Figure 3.50: Relative number distribution of the estimated energy required to form H I holes.

The amount of energy required for the hole formation is plotted with the normalized radial distribution of all the holes in Figure 3.51. The holes requiring the least amounts of energy are located in the outer regions of the galaxies. This is more easily seen when we observe the plots of individual galaxies (Figure 3.52), we see the energy required is higher in the inner disk of the galaxies than the outer parts. This might be due to higher gas density and higher star formation rate (SFR) near the center than the peripheral area. We find a rough consistency of this observation in 
DDO 43, DDO 46, DDO 52, DDO 53, DDO 87, DDO 154, DDO 155, F564-V3, IC 10, M81dwA etc.

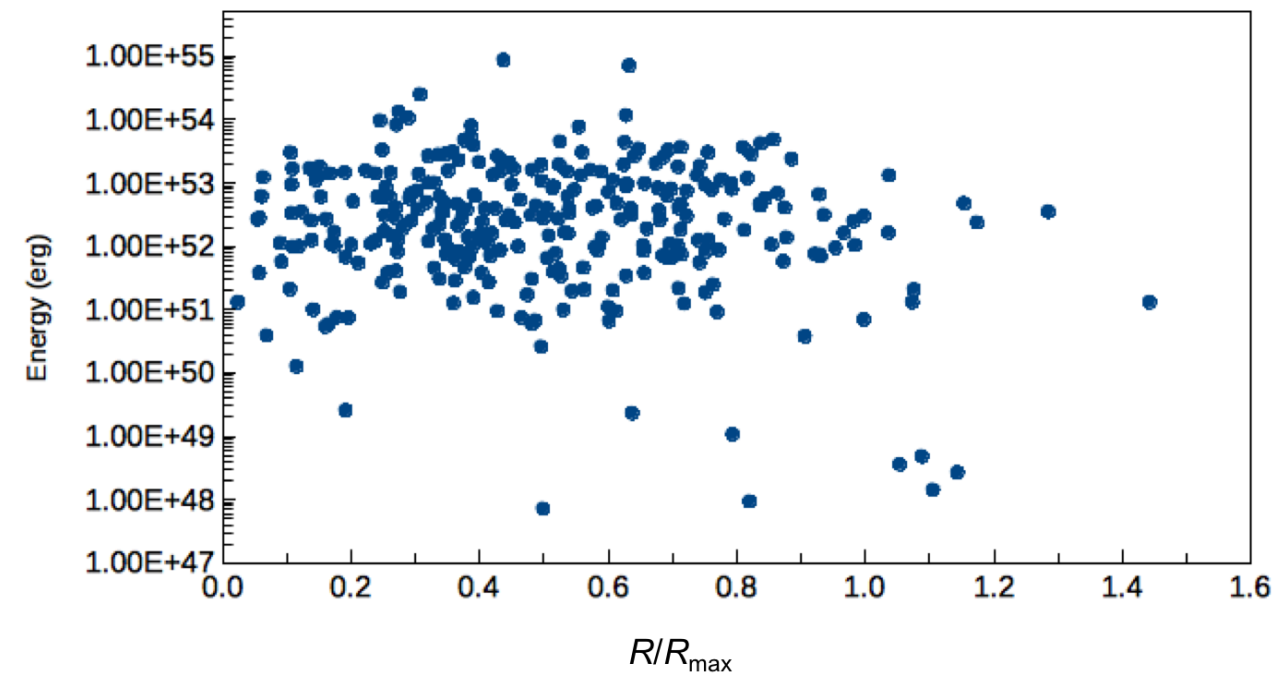

Figure 3.51: Estimated energy required to form $\mathrm{H}$ I holes of the entire sample with respect to their radial distributions. 


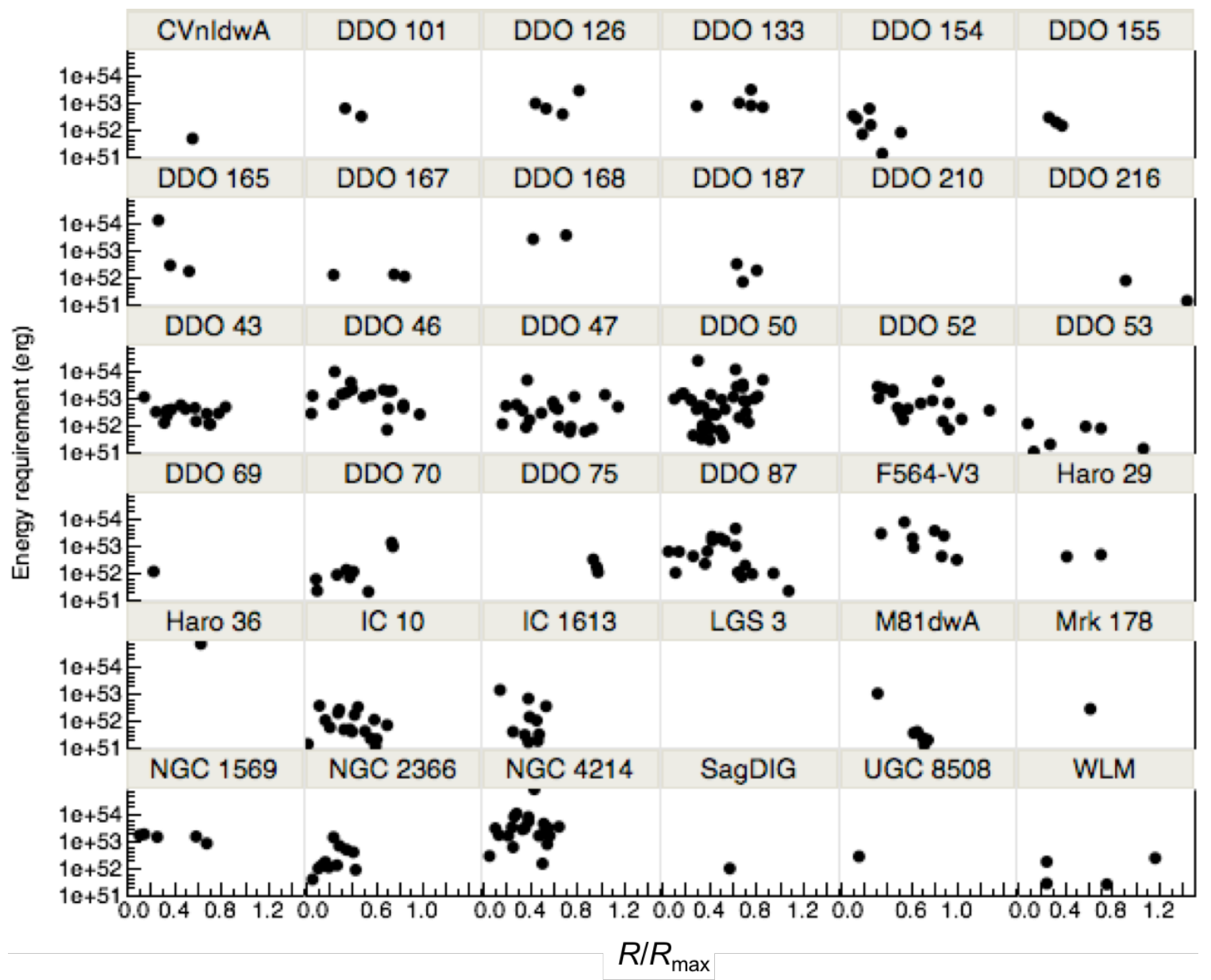

Figure 3.52: Estimated energy required to form H I holes vs. their normalized radial locations. 


\section{CHAPTER 4}

\section{Analysis}

\subsection{Porosity}

Porosity is defined as the ratio of the total area or volume covered by holes to the total area or volume covered by the H I associated with the galaxy [44]. Joseph Silk in 1997 first proposed from theory that there should be an anti-correlation between $\mathrm{H}$ I porosity and $\mathrm{H} \alpha$ surface brightness (star formation) [109]. Our expectation is that porosity might enhance star formation up to a certain limit. Assuming the holes are formed from stellar feedback, and they are continuously expanding, shells around the holes get thicker and thicker with time, resulting in an increase in temperature and pressure, which ultimately promotes the birth of the next generation of stars. Stellar feedback in the form of ionizing radiation, winds, and supernova explosions from these new born stars again creates holes and hence helps to form another generation of stars. The process goes on until the gas becomes too porous to get collected such that the star formation activity declines from that point i.e., the ISM becomes too porous for star formation to occur. Porosity is calculated in both two and three dimensions for our analysis, and the results are discussed below. The star formation activity is calculated using $\mathrm{H} \alpha$ emission and also from $\mathrm{FUV}$ emission. $\mathrm{H} \alpha$ is from stars formed in the past Myr; FUV is from stars formed in the past 100-200 Myr.

\subsubsection{Surface Porosity}

We calculated the ratio of the total area covered by cataloged holes to the total area

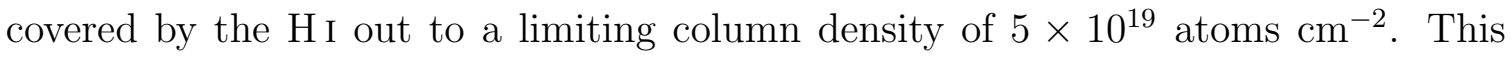
measures the surface porosity $\left(Q_{2 \mathrm{D}}\right)$ of the galaxy. We used AIPS tasks BLANK and 
ISPEC to estimate the area covered by H I to our column density limit. Calculation shows that most of the LITTLE THINGS galaxies have a surface porosity $\leq 15 \%$ (Table 4.1, Figure 4.1). The exceptions are M81DwA and F564-V3, with $Q_{2 \mathrm{D}}$ about $25 \%$ and $20 \%$ respectively. There are four galaxies in our sample with no $\mathrm{H} \alpha$ emission indicating little/no star formation within the past 10 Myr. We found highly porous M81DwA and F564-V3 are two of them. These two galaxies have very low FUV emission as well. Conversely, DDO 210 has very low porosity (0.8\%) with no star formation rate calculated from $\mathrm{H} \alpha$ emission and the second lowest FUV emission in the sample. LGS 3 also has no $\mathrm{H} \alpha$ emission and the lowest FUV emission but its surface porosity is about 7\%. Galaxies NGC 1569, NGC 2366, Haro 29, NGC 3738 and and IC 10 have very low surface porosity $(\leq 1.6 \%)$, but have high star formation rates whereas the galaxies NGC 4214 and DDO 50 have high SFR as well as high surface porosity ( $9 \%$ and $11 \%$ respectively). 
Table 4.1: Porosity and Star Formation Rate

\begin{tabular}{|c|c|c|c|c|c|c|c|c|}
\hline Galaxies & $\begin{array}{l}Q_{2 \mathrm{D}} \\
(\%)\end{array}$ & $\begin{array}{l}Q_{3 \mathrm{D}} \\
(\%)\end{array}$ & $\begin{array}{l}\log \mathrm{SFR}_{\mathrm{H} \alpha} \\
\left(M_{\odot} \mathrm{yr}^{-1}\right)\end{array}$ & $\begin{array}{c}\log \mathrm{SFR}_{\mathrm{FUV}} \\
\left(M_{\odot} \mathrm{yr}^{-1}\right)\end{array}$ & $\begin{array}{c}\log \mathrm{SFR}_{\text {Hole }} \\
\left(M_{\odot} \mathrm{yr}^{-1}\right)\end{array}$ & $\begin{array}{l}\text { SN per } \\
\text { Galaxy }\end{array}$ & $\begin{array}{l}\% \text { of Holes per } \\
\text { unit area in } R_{\mathrm{br}}\end{array}$ & $\begin{array}{l}\% \text { of } \mathrm{H} \alpha \text { in } \\
\text { diffused gas }\end{array}$ \\
\hline CVnIdwA & 0.50 & 0.03 & -2.64 & -2.47 & -4.11 & 35 & 0 & $\ldots$ \\
\hline DDO 43 & 6.54 & 0.53 & -2.12 & -1.83 & -1.95 & 4990 & 59 & 44 \\
\hline DDO 46 & 5.69 & 2.57 & -2.35 & -1.85 & -1.34 & 20620 & 79 & 56 \\
\hline DDO 47 & 7.59 & 1.32 & -1.98 & -1.63 & -1.64 & 10237 & 78 & 59 \\
\hline DDO 50 & 10.89 & 2.48 & -1.25 & -0.97 & -1.04 & 40779 & $\ldots$ & 37 \\
\hline DDO 52 & 5.60 & 2.44 & -2.53 & -1.83 & -1.37 & 19017 & 65 & $\ldots$ \\
\hline DDO 53 & 3.44 & 0.12 & -2.28 & -2.12 & -2.70 & 895 & 91 & 29 \\
\hline DDO 63 & 3.33 & $\ldots$ & -2.23 & -1.89 & $\ldots$ & $\ldots$ & 77 & $\ldots$ \\
\hline DDO 69 & 4.49 & 0.38 & -3.95 & -3.17 & -3.86 & 62 & 93 & 53 \\
\hline DDO 70 & 9.39 & 3.20 & -3.01 & -2.39 & -2.81 & 689 & 0 & 47 \\
\hline DDO 75 & 7.71 & 3.11 & -2.21 & -1.98 & -1.90 & 5686 & 0 & 51 \\
\hline DDO 87 & 12.35 & 4.25 & -2.35 & -1.95 & -1.73 & 8360 & 86 & 45 \\
\hline DDO 101 & 1.92 & 0.90 & -2.55 & -2.37 & -3.03 & 416 & 100 & 77 \\
\hline DDO 126 & 5.58 & 2.11 & -2.07 & -1.83 & -2.56 & 1227 & 0 & 37 \\
\hline DDO 133 & 5.74 & 2.67 & -2.26 & -1.93 & -2.48 & 1490 & 100 & 43 \\
\hline DDO 154 & 1.32 & 0.08 & -2.56 & -1.91 & -2.71 & 866 & 0 & 45 \\
\hline DDO 155 & 5.63 & 2.13 & -2.67 & $\ldots$ & -4.03 & 42 & 85 & 45 \\
\hline DDO 165 & 8.51 & 3.04 & -2.48 & $\ldots$ & -2.86 & 612 & 87 & 73 \\
\hline DDO 167 & 7.53 & 0.72 & -2.88 & -2.41 & -3.60 & 112 & 52 & 42 \\
\hline DDO 168 & 1.06 & 0.49 & -2.02 & -1.72 & -2.92 & 535 & 0 & 52 \\
\hline DDO 187 & 1.98 & 0.78 & -3.64 & -2.97 & -2.79 & 729 & 0 & 66 \\
\hline DDO 210 & 0.81 & 0.04 & -7.00 & -3.75 & -6.60 & 0 & $\ldots$ & $\cdots$ \\
\hline DDO 216 & 5.26 & 1.71 & -4.19 & -3.25 & -3.76 & 78 & 100 & 80 \\
\hline F564-V3 & 19.72 & 9.18 & -7.00 & -2.85 & -2.38 & 1855 & 0 & $\ldots$ \\
\hline Haro 29 & 1.30 & 0.15 & -1.41 & -1.68 & -2.72 & 861 & 75 & 6 \\
\hline Haro 36 & 3.96 & 1.85 & -1.80 & -1.37 & -1.91 & 5553 & 0 & $\cdots$ \\
\hline IC 10 & 1.54 & 0.00 & -1.62 & $\ldots$ & -1.40 & 17761 & 89 & 45 \\
\hline IC 1613 & 13.05 & 5.02 & -2.61 & -2.02 & -3.11 & 346 & 68 & $\ldots$ \\
\hline LGS 3 & 7.14 & 0.33 & -7.00 & -4.85 & -4.65 & 10 & 17 & $\cdots$ \\
\hline M81dwA & 24.56 & 8.41 & -7.00 & -2.94 & -3.19 & 287 & 0 & $\ldots$ \\
\hline Mrk 178 & 3.85 & 0.34 & -2.14 & -2.12 & -3.63 & 105 & 0 & 25 \\
\hline NGC 1569 & 0.48 & 0.04 & -0.22 & -0.49 & -1.32 & 21600 & 84 & 51 \\
\hline NGC 2366 & 1.04 & 0.07 & -0.97 & -0.98 & -1.95 & 5079 & 98 & 12 \\
\hline NGC 3738 & 1.26 & $\ldots$ & -1.45 & -1.25 & $\ldots$ & $\ldots$ & 48 & 30 \\
\hline NGC 4214 & 8.55 & 3.66 & -0.85 & -0.86 & -1.06 & 39125 & 86 & 44 \\
\hline SagDIG & 8.60 & 0.54 & -3.8 & -2.89 & -5.18 & 3 & 0 & $\ldots$ \\
\hline UGC 8508 & 1.81 & 0.17 & -2.78 & $\ldots$ & -3.35 & 198 & 84 & 46 \\
\hline WLM & 0.58 & 0.18 & -2.84 & -2.14 & -2.84 & 644 & 35 & 43 \\
\hline
\end{tabular}

\subsubsection{Volume Porosity}

For the volume porosity $\left(Q_{3 \mathrm{D}}\right)$, we calculated the ratio of the total volume of holes to that of the galaxy volume. We estimated the volume of the holes assuming they are spherical. For the estimation of the volume occupied by neutral hydrogen, we used the area from the surface porosity calculation and used the scale height as the third dimension. We assumed the scale height is constant throughout the H I disk. The volume porosity of most of the sample galaxies lies within the range $\leq 6 \%$ except for 
F564-V3 $(\approx 9 \%)$ and M81dwA $(\approx 8 \%)$ which have no recent star formation (no $\mathrm{H} \alpha$ emission). IC 10 has the lowest volume porosity (nearly equal to 0\%) among all the sample galaxies followed by CVnIdwA and NGC 1569. Values are given in Table 4.1 and plotted in Figure 4.1.

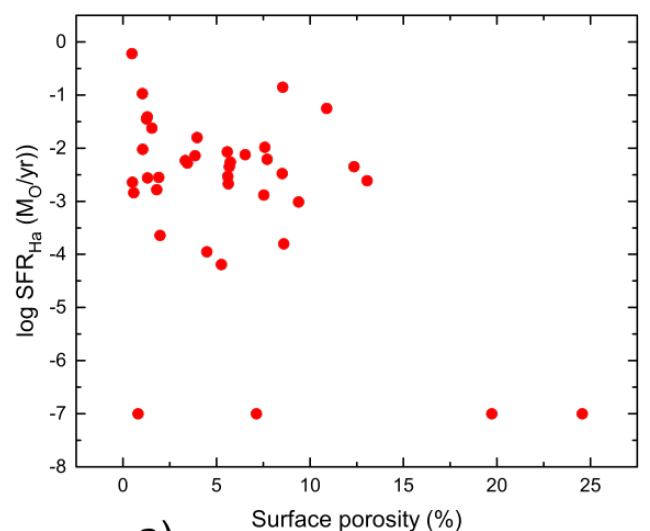

a)

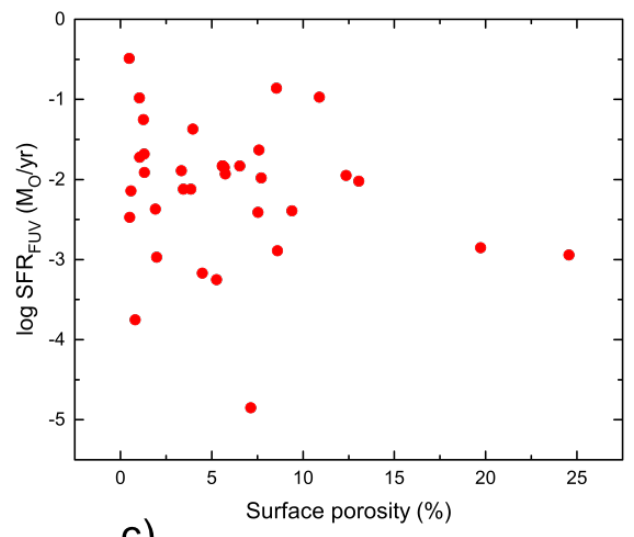

c)
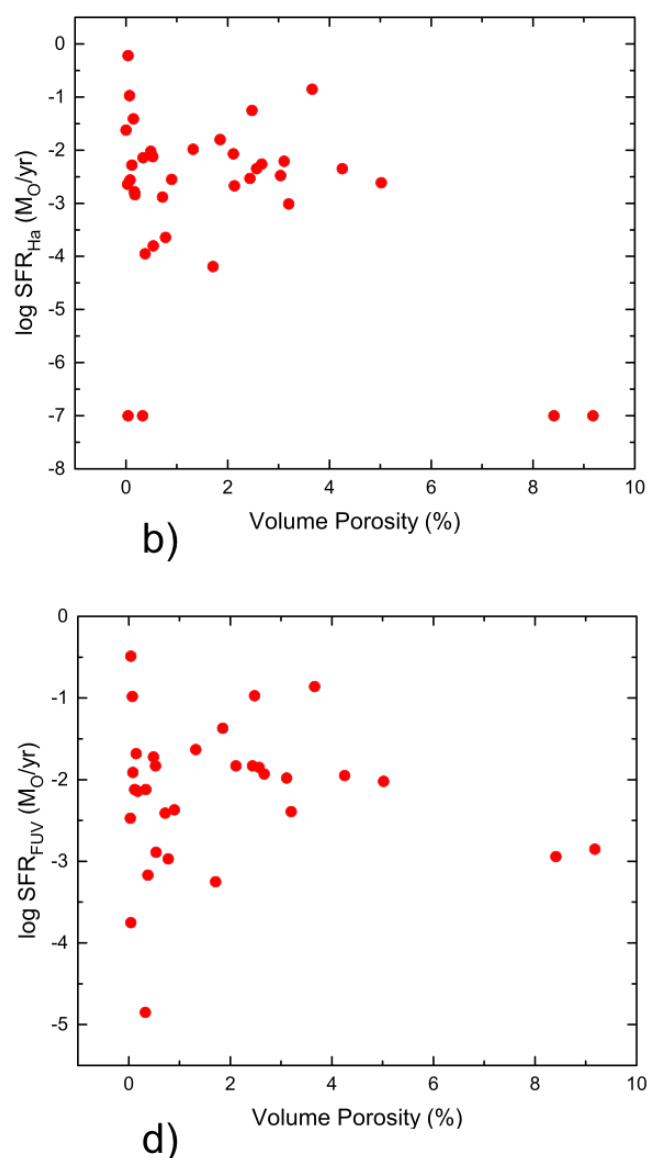

Figure 4.1: Porosity vs. star formation rate: a) surface porosity vs. the SFR calculated from $\mathrm{H} \alpha$ emission, b) volume porosity vs. the SFR calculated from $\mathrm{H} \alpha$ emission, c) surface porosity vs. the SFR calculated from FUV emission, and d) volume porosity vs. the SFR calculated from FUV emission. In Figures a and b, there is no star formation in the bottom four galaxies; $\mathrm{SFR}=-7$ is assigned just for plotting purposes.

From the results of the relation between the surface and volume porosity with star formation rates as shown in Figure 4.1, it is clearly seen that there is wide variation in star formation rate at low porosity. However, it is not clear whether high 
porosity is not favorable for star formation. According to the $\mathrm{H} \alpha$ tracer, two highly porous galaxies have no recent star formation at present; but some star formation is seen in these galaxies using the FUV tracer. Recall that $\mathrm{H} \alpha$ and FUV indicate star formation at different ages. H $\alpha$ shows SF in the past $10 \mathrm{Myr}$, FUV the past 100 to 200 Myr. So perhaps these galaxies had SF $\approx 100$ Myr ago, but are so porous that it shut off, hence no $\mathrm{H} \alpha$. This is speculative, but could be possible.

We don't see any obvious relation between the porosity and star formation, which might have two possible reasons. Either there is no specific correlation between H I porosity and star formation rate or our sample is not large enough to see the correlation.

\subsection{Star Formation Rate and Star Formation History}

Stellar feedback (supernova explosions and stellar radiation) is considered one of the most probable mechanisms for the origination of $\mathrm{H}$ I holes. The idea is that star clusters (such as OB associations) provide sufficient energy from stellar feedback to push out the gases around them which eventually creates a hole and a denser shell around the hole. Continued expansion increases the density and temperature of the shell originating another generation of stars [110]. This explanation is supported by Gil de Paz et al. [111] and Thilker et al. [112,113] using the GALEX Nearby Galaxies Survey. Based on this understanding, we expect the amount of energy required to form a hole to correlate with the amount of stars that produced it. To test this, we estimate the star formation rate needed to form a hole and look for an observational correlation with a star formation tracer such as $\mathrm{H} \alpha$.

We estimated the number of total supernovae required to create each hole using the average energy produced during a supernova explosion $\left(\approx 10^{51}\right)$ erg as in McCray and Kafatos (1987) [57]. We also assumed the average lifetime of a hole to be $\approx 60$ 
Myr, and that all the holes are formed by Type II supernovae of stars with masses $\gtrsim 8 M_{\odot}[33]$.

The plot of the number of supernovae required to form the holes versus the kinetic age of the holes for 36 LITTLE THINGS galaxies is shown in Figure 4.2 which shows the kinetic age of the holes versus the number of supernovae (SNe) required to form each hole. To calculate the number of SNe, we have used $10^{51}$ erg [57] as the average energy released by one supernova. There is no observed correlation between age and number of SNe, but this plot also provides a rough estimate of the star formation history of each galaxy. As we see, DDO 69, DDO 187, IC 10, NGC 1569 and WLM have very recent star formation activities, $\approx 10 \mathrm{Myr}$ or less. For some of the galaxies like DDO 46, DDO 50, NGC 2366, and NGC 4214, the SFH is more or less constant throughout the period.

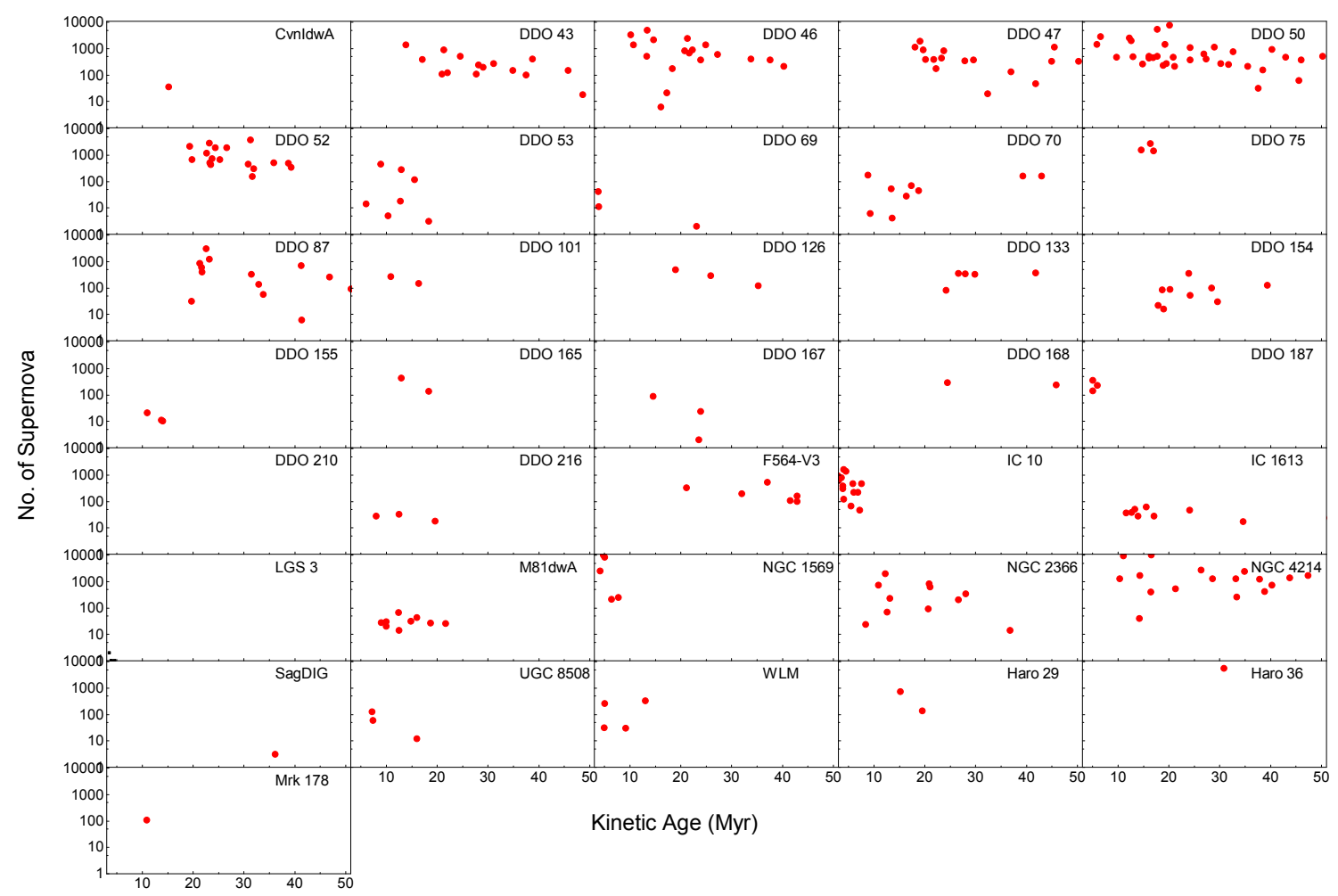

Figure 4.2: Kinetic age of the $\mathrm{H}$ I holes vs. the number of supernovae required to form them. 
Using the supernovae history estimated from the holes, we can estimate the supernovae rate (SNR). We calculated the SNR for each hole using the current expansion velocity, the radius of the hole, and the number of supernovae required to form the holes. The supernovae rate is proportional to the star formation rate (SFR) for stars with masses $\gtrsim 8 M_{\odot}$. The constant of proportionality is calculated by integrating the Salpeter initial mass function

$$
N=\int_{0.1}^{120} A M^{-2.35} d M
$$

where $A$ is the constant of proportionality, $M$ is the mass of the star, and $N$ is the number of stars formed. The stellar mass limits $\left(0.1\right.$ to $\left.120 M_{\odot}\right)$ are taken as in Bagetakos et al. [33]. From calculation we obtain the relation between the total SFR (including stars with masses less than $8 M_{\odot}$ ) and the SFR as estimated from the number of SNe required to create the holes as

$$
\operatorname{SFR}_{\text {Holes }} \approx 134 \operatorname{SFR}\left(M_{\odot} \gtrsim 8\right)
$$

Since the hole formation process is ongoing and we are calculating the star formation rate from the holes using their properties observed present, the calculated value of $\mathrm{SFR}_{\text {Holes }}$ gives the lower limit of the star formation rate. Note that the upper limit of the age of the holes used in the calculation underestimates the $\mathrm{SFR}_{\text {Holes }}$. The calculated values of SFR from the holes are in Table 4.1.

We can now test whether the star formation activity required to the form the holes is consistent with the amount of recent star formation as indicated by $\mathrm{H} \alpha$ and FUV emission. In Figure 4.3, the star formation rate estimated from the holes is plotted with the star formation rates measured from $\mathrm{H} \alpha$ and FUV data. In general, the relation shows that the holes are consistent with being from star formation. 

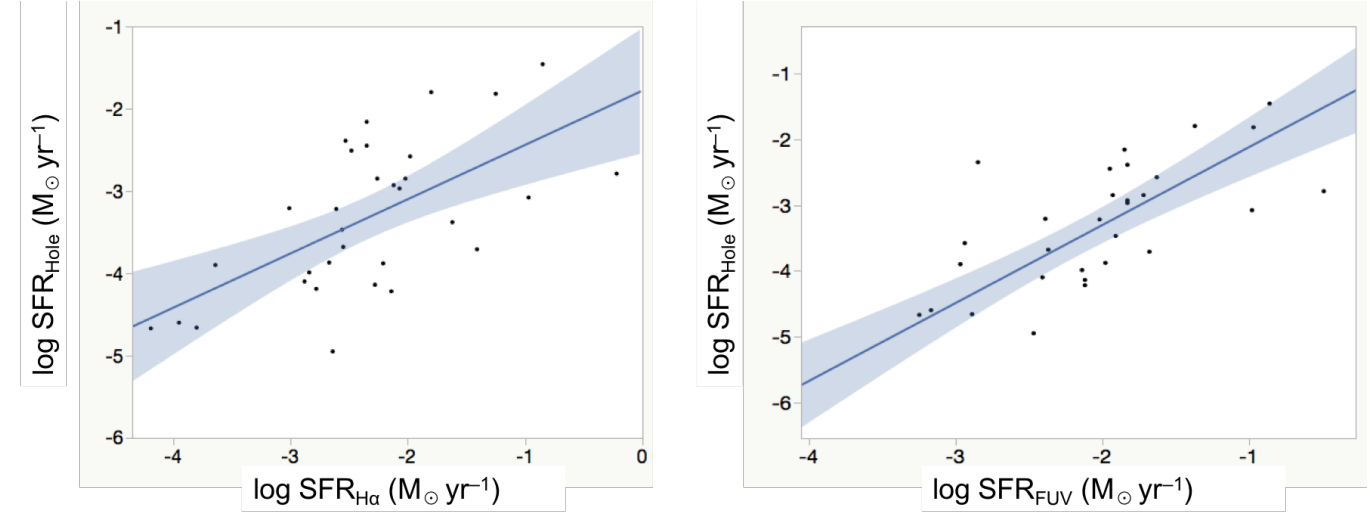

Figure 4.3: The star formation rate estimated from the energy required to form the $\mathrm{H}$ I holes vs. the stellar star formation rates $\mathrm{H} \alpha$ (left) and FUV (right). The solid line is the best fit linear regression line. The shaded region shows the confidence limits $(95 \%)$ for the expected value. 


\section{CHAPTER 5}

\section{Conclusions and Future Work}

\subsection{Conclusions}

In this research, we followed a systematic and consistent procedure to identify and catalog $\mathrm{H}$ I holes in dwarf irregular galaxies, and analyzed their properties. This is part of the work of the LITTLE THINGS project which studies 41 nearby dwarf galaxies including 4 blue compact dwarfs.

We searched for holes in the integrated natural-weighted and robust-weighted H I flux density maps and data cubes using the KARMA visualization software and performed a visual inspection following specific criteria. Our initial catalog contains more than 1000 candidate holes. From those structures we selected 306 high quality H I holes. We measured their observed properties and calculated various others, such as the kinetic ages of the holes, the mass required to fill the holes, the energy required to form those structures, and other properties.

We could measure the expansion velocity of the Type 2 and Type 3 holes, which ranges from 5 to $30 \mathrm{~km} \mathrm{~s}^{-1}$. The expansion rates of Type 1 holes are not measurable because they are completely broken in position-velocity diagrams. The rotation velocities, range from $6 \mathrm{~km} \mathrm{~s}^{-1}$ for SagDIG to $77 \mathrm{~km} \mathrm{~s}^{-1}$ for NGC 4214 . The extent of the gas disk is defined as the radius at which the rotation velocity is a maximum in the pv diagram. The smallest gas disk measured from the pv diagrams along the major axis, is for DDO 187 with $R_{\max }=0.5 \mathrm{kpc}$; the largest is for DDO 50 with $R_{\max }=6.7 \mathrm{kpc}$. The H I scale heights of the galaxies range from $64 \mathrm{pc}$ to 653 pc. Most of the holes are midway in the disks of the galaxies, with diameters $\lesssim 0.5$ kpc. The upper limits of the kinetic ages of the holes vary from 1 Myr to $127 \mathrm{Myr}$. 
Using information from the catalog, we calculated the surface $\left(Q_{2 \mathrm{D}}\right)$ and volume $\left(Q_{3 \mathrm{D}}\right)$ porosities of the $\mathrm{H}$ I gas, as defined by the percentage of the surface area (or volume) of the H I content of the galaxy containing holes. From the study of porosity we found that most of the galaxies have $Q_{2 \mathrm{D}} \leq 15 \%$ and $Q_{3 \mathrm{D}} \leq 6 \%$. The four galaxies with no recent $\mathrm{SF}$ as measured by $\mathrm{H} \alpha$ have either very low, or very high porosities. Galaxies with low-to-intermediate porosities exhibit a wide range of star formation activity, however.

We do not find a distinct level of porosity that corresponds to a cut-off in star formation as predicted in Silk [109]. It may be that our sample is too small to definitively detect any SF cutoff at high porosities. The lack of recent SF in the four galaxies with either the highest or lowest porosities is suggestive, however. The two galaxies with the highest porosities and no recent SF (but low levels of intermediateage SF) are consistent with the idea that at some point, the $\mathrm{H}$ I becomes too full of holes to support new regions of star formation. The other two galaxies with no star formation and extremely low (volume) porosities may reflect the need for recent star formation to form structure in the H I.

These results are also consistent with the relation we find between the star formation rate estimated from the energy required to create a hole (from $\mathrm{SNe}$, and therefore related to $\mathrm{SF}$ ) and the star formation rates measured from $\mathrm{H} \alpha$ and FUV. This indicates that the holes are consistent with a star formation origin. From this result, we conclude that stellar feedback can be considered as an important factor in creating $\mathrm{H}$ I holes, as postulated by Weaver et al., Cash et al., Ott et al., Weisz et al., and Cannon et al. $[18,55,56,59,61]$. However, the uncertainty seen in the plots shown in our work support a more complex idea of the relation between star formation and the atomic gas in small galaxies. It may be possible that these structures formed because of the combined result of two or more phenomena such as stellar feedback, 
turbulence, thermal and gravitational instabilities, gamma ray bursts and/or high velocity cloud impacts, as suggested by the numerical simulations of Dib and Burkert, and Vorobyov and Basu $[69,71]$.

\subsection{Future Work}

The catalog will be used in several upcoming projects, both by us and in collaboration with other members of the LITTLE THINGS team. Our immediate future work is to study the H II regions associated with $\mathrm{H}$ I holes and their relation with the diffuse ionized gas (DIG) as suggested by Martin (1997) [114]. We are using the values for

the H II regions in the galaxies as in Youngblood and Hunter (1999) [115]. We will estimate the H I mass fraction that is in the shells and study its correlation with the star formation rate.

Another project will be to study the density gradient along the edges of the holes to determine the hole formation mechanism. If the holes are formed by stellar feedback in the form of radiation pressure they will have a gradual change in density at the hole edges. The holes formed by mechanical energy from supernova explosion should have steeper changes in density [116]. We will also study the hole formation mechanism by calculating the stellar age gradient in the holes as in Dopita et al. (1985) [117]. When the formation mechanism is known, we can calculate the energy required to form the hole more precisely.

The study of the relation between porosity and the $\mathrm{H}$ I column density and $\mathrm{H}$ I velocity dispersion will be another future work project. 


\section{Bibliography}

[1] E. P. Hubble. Extragalactic nebulae. The Astrophysical Journal, 64, December 1926.

[2] I. Bagetakos. Shells, Bubbles and Holes: The Porosity of the Interstellar Medium in Galaxies. PhD thesis, University of Hertfordshire, 2012.

[3] I. D. Karachentsev, V. E. Karachentseva, W. K. Huchtmeier, and D. I. Makarov. A Catalog of Neighboring Galaxies. The Astronomical Journal, 127:2031-2068, April 2004.

[4] D. R. Weisz. The history of star formation in nearby dwarf galaxies. PhD thesis, University of Minnesota, 2010.

[5] H. Mo, F. Van den Bosch, and S. White. Galaxy formation and evolution. Cambridge University Press, 2010.

[6] T. Sawala, C. Scannapieco, and S. White. Local Group dwarf galaxies: nature and nurture. Monthly Notices of the Royal Astronomical Society, 420:1714-1730, February 2012.

[7] G. Kauffmann, S. D. M. White, and B. Guiderdoni. The Formation and Evolution of Galaxies Within Merging Dark Matter Haloes. Monthly Notices of the Royal Astronomical Society, 264:201, September 1993.

[8] G. Kauffmann, S. Charlot, and S. D. M. White. Detection of strong evolution in the population of early-type galaxies. Monthly Notices of the Royal Astronomical Society, 283:L117-L122, December 1996.

[9] E. Tolstoy, V. Hill, and M. Tosi. Star-Formation Histories, Abundances, and Kinematics of Dwarf Galaxies in the Local Group. Annual Review of Astronomy E Astrophysics, 47:371-425, September 2009.

[10] D. A. Hunter and J. D. Plummer. Sextans A: A Case Study of Star Formation and Gas Densities in Irregular Galaxies. The Astrophysical Journal, 462:732, May 1996.

[11] D. A. Hunter, B. G. Elmegreen, and A. L. Baker. The Relationship between Gas, Stars, and Star Formation in Irregular Galaxies: A Test of Simple Models. The Astrophysical Journal, 493:595-612, January 1998.

[12] L. L. Cowie, A. Songaila, E. M. Hu, and J. G. Cohen. New Insight on Galaxy Formation and Evolution From Keck Spectroscopy of the Hawaii Deep Fields. The Astronomical Journal, 112:839, September 1996. 
[13] E. D. Skillman. Neutral Hydrogen in Dwarf Galaxies. In E. D. Skillman, editor, The Minnesota Lectures on Extragalactic Neutral Hydrogen, volume 106 of Astronomical Society of the Pacific Conference Series, page 208, 1996.

[14] R. C. Dohm-Palmer, E. D. Skillman, A. Saha, E. Tolstoy, M. Mateo, J. Gallagher, J. Hoessel, C. Chiosi, and R. J. Dufour. The Dwarf Irregular Galaxy Sextans A. II. Recent Star Formation History. The Astronomical Journal, 114:2527, December 1997.

[15] M. L. Mateo. Dwarf Galaxies of the Local Group. Annual Review of Astronomy E Astrophysics, 36:435-506, 1998.

[16] S. van den Bergh. The Galaxies of the Local Group. Cambridge, May 2000.

[17] D. Thomas, C. Maraston, R. Bender, and C. Mendes de Oliveira. The Epochs of Early-Type Galaxy Formation as a Function of Environment. The Astrophysical Journal, 621:673-694, March 2005.

[18] D. R. Weisz, E. D. Skillman, J. M. Cannon, A. E. Dolphin, R. C. Kennicutt, Jr., J. Lee, and F. Walter. Does Stellar Feedback Create H i Holes? A Hubble Space Telescope/Very Large Array Study of Holmberg II. The Astrophysical Journal, 704:1538-1569, October 2009.

[19] R. C. Kennicutt, Jr. The star formation law in galactic disks. The Astrophysical Journal, 344:685-703, September 1989.

[20] D. A. Hunter. Local properties of star-forming regions in irregular galaxies. The Astrophysical Journal, 260:81-103, September 1982.

[21] R. Sancisi, F. Fraternali, T. Oosterloo, and T. van der Hulst. Cold gas accretion in galaxies. Astronomy \& Astrophysics Reviews, 15:189-223, June 2008.

[22] G. Kauffmann, C. Li, and T. M. Heckman. The accretion of gas on to galaxies as traced by their satellites. Monthly Notices of the Royal Astronomical Society, 409:491-499, December 2010.

[23] G. Efstathiou. Suppressing the formation of dwarf galaxies via photoionization. Monthly Notices of the Royal Astronomical Society, 256:43P-47P, May 1992.

[24] J. F. Navarro and M. Steinmetz. The Effects of a Photoionizing Ultraviolet Background on the Formation of Disk Galaxies. The Astrophysical Journal, 478:13-28, March 1997.

[25] M. Dijkstra, Z. Haiman, M. J. Rees, and D. H. Weinberg. Photoionization Feedback in Low-Mass Galaxies at High Redshift. The Astrophysical Journal, 601:666-675, February 2004. 
[26] N. Y. Gnedin. Effect of Reionization on Structure Formation in the Universe. The Astrophysical Journal, 542:535-541, October 2000.

[27] A. A. Cole, E. D. Skillman, E. Tolstoy, J. S. Gallagher, III, A. Aparicio, A. E. Dolphin, C. Gallart, S. L. Hidalgo, A. Saha, P. B. Stetson, and D. R. Weisz. Leo A: A Late-blooming Survivor of the Epoch of Reionization in the Local Group. The Astrophysical Journal, Letters, 659:L17-L20, April 2007.

[28] B. T. Draine. Interstellar Dust Grains. Annual Review of Astronomy \& Astrophysics, 41:241-289, 2003.

[29] J. Hartmann. Investigations on the spectrum and orbit of delta Orionis. The Astrophysical Journal, 19, May 1904.

[30] S. R. Kulkarni and C. Heiles. Neutral hydrogen and the diffuse interstellar medium, pages 95-153. Springer Science \& Business Media, 1988.

[31] A. G. G. M. Tielens. The Physics and Chemistry of the Interstellar Medium. Cambridge University Press, August 2005.

[32] S. Kwok. Physics and Chemistry of the Interstellar Medium. University Science Books, 2007.

[33] I. Bagetakos, E. Brinks, F. Walter, W. J. G. de Blok, A. Usero, A. K. Leroy, J. W. Rich, and R. C. Kennicutt, Jr. The Fine-scale Structure of the Neutral Interstellar Medium in Nearby Galaxies. The Astronomical Journal, 141:23, January 2011.

[34] H. I. Ewen and E. M. Purcell. Observation of a Line in the Galactic Radio Spectrum: Radiation from Galactic Hydrogen at 1,420 Mc./sec. Nature, 168:356, September 1951.

[35] C. A. Muller and J. H. Oort. Observation of a Line in the Galactic Radio Spectrum: The Interstellar Hydrogen Line at 1,420 Mc./sec., and an Estimate of Galactic Rotation. Nature, 168:357-358, September 1951.

[36] K. G. Jansky. Electrical phenomena that apparently are of interstellar origin. Popular Astronomy, 41:548, December 1933.

[37] K. G. Jansky. Radio Waves from Outside the Solar System. Nature, 132:66, July 1933.

[38] G. Reber. Notes: Cosmic Static. The Astrophysical Journal, 91:621-624, June 1940 .

[39] A. Singhal. The connection between galaxy kinematics and $H_{\mathrm{I}}$ line widths as applied to the distance scale. PhD thesis, University of Virginia, 2008. 
[40] B. E. Westerlund and D. S. Mathewson. Supernova remnants in the large Magellanic Cloud. Monthly Notices of the Royal Astronomical Society, 131:371, 1966.

[41] J. V. Hindman. A high resolution study of the distribution and motions of neutral hydrogen in the Small Cloud of Magellan. Australian Journal of Physics, 20:147, 1967.

[42] H. Weaver and D. R. W. Williams. The Berkeley low-latitude survey of neutral hydrogen Part I. Profiles. Astronomy \&3 Astrophysics, Supplement, 8:1, 1973.

[43] C. Heiles. An almost complete survey of 21-centimeter line radiation for galactic latitudes of at least $10 \mathrm{deg}$. VI - Energetic expanding H i shells. The Astrophysical Journal, Letters, 208:L137-L139, September 1976.

[44] D. P. Cox and B. W. Smith. Large-Scale Effects of Supernova Remnants on the Galaxy: Generation and Maintenance of a Hot Network of Tunnels. The Astrophysical Journal, Letters, 189:L105, May 1974.

[45] C. F. McKee and J. P. Ostriker. A theory of the interstellar medium - Three components regulated by supernova explosions in an inhomogeneous substrate. The Astrophysical Journal, 218:148-169, November 1977.

[46] C. Heiles. H I shells and supershells. The Astrophysical Journal, 229:533-537, April 1979.

[47] C. Heiles. H I shells, supershells, shell-like objects, and 'worms'. The Astrophysical Journal, Supplement, 55:585-595, August 1984.

[48] E. M. Hu. High latitude H i shells in the Galaxy. I. The Astrophysical Journal, 248:119-127, August 1981.

[49] E. Brinks and E. Bajaja. A high resolution hydrogen-line survey of Messier 31. III - H I holes in the interstellar medium. Astronomy \& Astrophysics, 169:14-42, November 1986.

[50] D. Puche, D. Westpfahl, E. Brinks, and J.-R. Roy. Holmberg II - A laboratory for studying the violent interstellar medium. The Astronomical Journal, 103:1841-1858, June 1992.

[51] S. Kim, M. A. Dopita, L. Staveley-Smith, and M. S. Bessell. H i Shells in the Large Magellanic Cloud. The Astronomical Journal, 118:2797-2823, December 1999.

[52] F. Walter and E. Brinks. Holes and Shells in the Interstellar Medium of the Nearby Dwarf Galaxy IC 2574. The Astronomical Journal, 118:273-301, July 1999. 
[53] Y. H. Chu. Massive stars as cosmic engines (iau symp. 250), ed, 2008.

[54] S. R. Warren, D. R. Weisz, E. D. Skillman, J. M. Cannon, J. J. Dalcanton, A. E. Dolphin, R. C. Kennicutt, Jr., B. Koribalski, J. Ott, A. M. Stilp, S. D. Van Dyk, F. Walter, and A. A. West. The Formation of Kiloparsec-scale H I Holes in Dwarf Galaxies. The Astrophysical Journal, 738:10, September 2011.

[55] R. Weaver, R. McCray, J. Castor, P. Shapiro, and R. Moore. Interstellar bubbles. II - Structure and evolution. The Astrophysical Journal, 218:377-395, December 1977.

[56] W. Cash, P. Charles, S. Bowyer, F. Walter, G. Garmire, and G. Riegler. The Xray superbubble in Cygnus. The Astrophysical Journal, Letters, 238:L71-L76, June 1980 .

[57] R. McCray and M. Kafatos. Supershells and propagating star formation. The Astrophysical Journal, 317:190-196, June 1987.

[58] G. Tenorio-Tagle and P. Bodenheimer. Large-scale expanding superstructures in galaxies. Annual Review of Astronomy \& Astrophysics, 26:145-197, 1988.

[59] J. Ott, F. Walter, E. Brinks, S. D. Van Dyk, B. Dirsch, and U. Klein. Evidence for BlowOut in the Low-Mass Dwarf Galaxy Holmberg I. The Astronomical Journal, 122:3070-3091, December 2001.

[60] C. E. Simpson, D. A. Hunter, and P. M. Knezek. DDO 88: A Galaxy-sized Hole in the Interstellar Medium. The Astronomical Journal, 129:160-177, January 2005 .

[61] J. M. Cannon, H. P. Most, E. D. Skillman, D. R. Weisz, D. Cook, A. E. Dolphin, R. C. Kennicutt, Jr., J. Lee, A. Seth, F. Walter, and S. R. Warren. The M81 Group Dwarf Irregular Galaxy DDO 165. I. High-velocity Neutral Gas in a Post-starburst System. The Astrophysical Journal, 735:35, July 2011.

[62] G. Tenorio-Tagle. The collision of clouds with the galactic disk. Astronomy 86 Astrophysics, 94:338, February 1981.

[63] R. J. Rand and J. M. Stone. Modeling the H I Supershell in the Edge-on-Galaxy NGC 4631 as an Energetic HVC Impact. The Astronomical Journal, 111:190, January 1996.

[64] A. Santillán, J. Franco, M. Martos, and J. Kim. The Collisions of High-Velocity Clouds with a Magnetized Gaseous Galactic Disk. The Astrophysical Journal, 515:657-668, April 1999.

[65] S. D. Murray and D. N. C. Lin. Energy Dissipation in Multiphase Infalling Clouds in Galaxy Halos. The Astrophysical Journal, 615:586-594, November 2004 . 
[66] Y. N. Efremov, B. G. Elmegreen, and P. W. Hodge. Giant Shells and Stellar Arcs as Relics of Gamma-Ray Burst Explosions. The Astrophysical Journal, Letters, 501:L163-L165, July 1998.

[67] A. Loeb and R. Perna. Are Hi Supershells the Remnants of Gamma-Ray Bursts? The Astrophysical Journal, Letters, 503:L35-L37, August 1998.

[68] R. Perna and J. Raymond. Testing the Multiple Supernovae versus Gamma-Ray Burst Scenarios for Giant H I Supershells. The Astrophysical Journal, 539:706711, August 2000.

[69] S. Dib and A. Burkert. On the Origin of the H I Holes in the Interstellar Medium of Dwarf Irregular Galaxies. The Astrophysical Journal, 630:238-249, September 2005.

[70] M. Relaño, J. E. Beckman, O. Daigle, and C. Carignan. An evolutionary sequence of expanding hydrogen shells in galaxy discs. Astronomy $\& 3$ Astrophysics, 467:1117-1123, June 2007.

[71] E. I. Vorobyov and S. Basu. Numerical simulations of expanding supershells in dwarf irregular galaxies. II. Formation of giant H I rings. Astronomy \& Astrophysics, 431:451-464, February 2005.

[72] J. Castor, R. McCray, and R. Weaver. Interstellar bubbles. The Astrophysical Journal, Letters, 200:L107-L110, September 1975.

[73] J. E. Dyson. Stellar wind bubbles in H II regions. Astronomy \& Astrophysics, 59:161-165, July 1977.

[74] M. S. Oey. Towards resolving the evolution of multi-supernova superbubbles. In B. G. Elmegreen and J. Palous, editors, Triggered Star Formation in a Turbulent ISM, volume 237 of IAU Symposium, pages 106-113, 2007.

[75] R. C. Kennicutt, Jr., F. Bresolin, D. J. Bomans, G. D. Bothun, and I. B. Thompson. Large scale structure of the ionized gas in the magellanic clouds. The Astronomical Journal, 109:594-604, February 1995.

[76] C. L. Martin. The Impact of Star Formation on the Interstellar Medium in Dwarf Galaxies. II. The Formation of Galactic Winds. The Astrophysical Journal, 506:222-252, October 1998.

[77] F. Walter, A. Weiss, C. Martin, and N. Scoville. The Interacting Dwarf Galaxy NGC 3077: The Interplay of Atomic and Molecular Gas with Violent Star Formation. The Astronomical Journal, 123:225-237, January 2002.

[78] Y. Pidopryhora, F. J. Lockman, and J. C. Shields. The Ophiuchus Superbubble: A Gigantic Eruption from the Inner Disk of the Milky Way. The Astrophysical Journal, 656:928-942, February 2007. 
[79] P. Frick, R. Beck, E. M. Berkhuijsen, and I. Patrickeyev. Scaling and correlation analysis of galactic images. Monthly Notices of the Royal Astronomical Society, 327:1145-1157, November 2001.

[80] A. Hughes, T. Wong, R. Ekers, L. Staveley-Smith, M. Filipovic, S. Maddison, Y. Fukui, and N. Mizuno. A multiresolution analysis of the radio-FIR correlation in the Large Magellanic Cloud. Monthly Notices of the Royal Astronomical Society, 370:363-379, July 2006.

[81] F. S. Tabatabaei, R. Beck, M. Krause, E. M. Berkhuijsen, R. Gehrz, K. D. Gordon, J. L. Hinz, R. Humphreys, K. McQuinn, E. Polomski, G. H. Rieke, and C. E. Woodward. A multi-scale study of infrared and radio emission from Scd galaxy M 33. Astronomy \& Astrophysics, 466:509-519, May 2007.

[82] G. Dumas, E. Schinnerer, F. S. Tabatabaei, R. Beck, T. Velusamy, and E. Murphy. The Local Radio-IR Relation in M51. The Astronomical Journal, 141:41, February 2011.

[83] L. Deharveng, A. Zavagno, F. Schuller, J. Caplan, M. Pomarès, and C. De Breuck. Star formation around RCW 120, the perfect bubble. Astronomy \& Astrophysics, 496:177-190, March 2009.

[84] D. A. Hunter, D. Ficut-Vicas, T. Ashley, E. Brinks, P. Cigan, B. G. Elmegreen, V. Heesen, K. A. Herrmann, M. Johnson, S.-H. Oh, M. P. Rupen, A. Schruba, C. E. Simpson, F. Walter, D. J. Westpfahl, L. M. Young, and H.-X. Zhang. Little Things. The Astronomical Journal, 144:134, November 2012.

[85] D. A. Hunter and B. G. Elmegreen. Star Formation Properties of a Large Sample of Irregular Galaxies. The Astronomical Journal, 128:2170-2205, November 2004 .

[86] D. A. Hunter and B. G. Elmegreen. Broadband Imaging of a Large Sample of Irregular Galaxies. The Astrophysical Journal, Supplement, 162:49-79, January 2006 .

[87] D. A. Thilker, R. Braun, and R. M. Walterbos. Expanding H I shells in NGC 2403. First results from an automated object recognition package. Astronomy E Astrophysics, 332:429-448, April 1998.

[88] S. Mashchenko and N. St-Louis. Automatic Shell Detection in CGPS Data. In A. F. J. Moffat and N. St-Louis, editors, Interacting Winds from Massive Stars, volume 260 of Astronomical Society of the Pacific Conference Series, page 65, 2002 .

[89] A. Daigle, G. Joncas, M. Parizeau, and M.-A. Miville-Deschênes. Automatic Detection of Expanding H I Shells Using Artificial Neural Networks. Publications of the ASP, 115:662-674, June 2003. 
[90] S. Ehlerová and J. Palouš. H I shells in the outer Milky Way. Astronomy $\&$ Astrophysics, 437:101-112, July 2005.

[91] S. Ehlerová and J. Palouš. H I shells in the Leiden/Argentina/Bonn H I survey. Astronomy \& Astrophysics, 550:A23, February 2013.

[92] R. Gooch. Karma: a Visualization Test-Bed. In G. H. Jacoby and J. Barnes, editors, Astronomical Data Analysis Software and Systems V, volume 101 of Astronomical Society of the Pacific Conference Series, page 80, 1996.

[93] R. A. Chevalier. The Evolution of Supernova Remnants. Spherically Symmetric Models. The Astrophysical Journal, 188:501-516, March 1974.

[94] L. van Zee. Discovery of an Extremely Metal-Poor Galaxy: Optical Spectroscopy of UGCA 292. The Astrophysical Journal, Letters, 543:L31-L34, November 2000.

[95] C. E. Simpson, D. A. Hunter, and T. E. Nordgren. DDO 43: A Prototypical Dwarf Irregular Galaxy? The Astronomical Journal, 130:1049-1064, September 2005 .

[96] F. Walter and E. Brinks. The Neutral Interstellar Medium of the Dwarf Irregular Galaxy DDO 47 and Its Companion. The Astronomical Journal, 121:3026-3040, June 2001.

[97] S. S. Kaisin and I. D. Karachentsev. Canes Venatici I cloud of galaxies seen in the $\mathrm{H} \alpha$ line. Astronomy $\&$ Astrophysics, 479:603-624, February 2008.

[98] G. L. Hoffman, E. E. Salpeter, and N. J. Carle. The Fine Structure and Outskirts of DDO 154. The Astronomical Journal, 122:2428-2443, November 2001.

[99] E. M. Wilcots and B. W. Miller. The Kinematics and Distribution of H I in IC 10. The Astronomical Journal, 116:2363-2394, November 1998.

[100] T. A. Lozinskaya, A. V. Moiseev, and N. Y. Podorvanyuk. Detailed Kinematic Study of the Ionized and Neutral Gas in the Complex of Star Formation in the Galaxy IC 1613. Astronomy Letters, 29:77-90, February 2003.

[101] S. Silich, T. Lozinskaya, A. Moiseev, N. Podorvanuk, M. Rosado, J. Borissova, and M. Valdez-Gutierrez. On the neutral gas distribution and kinematics in the dwarf irregular galaxy IC 1613. Astronomy \&6 Astrophysics, 448:123-131, March 2006.

[102] J. M. Stil and F. P. Israel. Neutral hydrogen in dwarf galaxies. I. The spatial distribution of H I. Astronomy \& Astrophysics, 389:29-41, July 2002. 
[103] K. E. Dellenbusch, J. S. Gallagher, III, P. M. Knezek, and A. G. Noble. Deep Optical Imaging of Starbursting "Transition" Dwarf Galaxies. The Astronomical Journal, 135:326-332, January 2008.

[104] L. Angeretti, M. Tosi, L. Greggio, E. Sabbi, A. Aloisi, and C. Leitherer. The Complex Star Formation History of NGC 1569. The Astronomical Journal, 129:2203-2216, May 2005.

[105] S. S. Larsen, L. Origlia, J. Brodie, and J. S. Gallagher. Anatomy of a young massive star cluster: NGC 1569-B. Monthly Notices of the Royal Astronomical Society, 383:263-276, January 2008.

[106] G. de Vaucouleurs, A. de Vaucouleurs, H. G. Corwin, Jr., R. J. Buta, G. Paturel, and P. Fouque. Book-Review - Third Reference Catalogue of Bright Galaxies. Sky $\&$ Telescope, 82:621, December 1991.

[107] C. E. Simpson, D. A. Hunter, T. E. Nordgren, E. Brinks, B. G. Elmegreen, T. Ashley, R. Lynds, V. J. McIntyre, E. J. O'Neil, G. Östlin, D. J. Westpfahl, and E. M. Wilcots. VII Zw 403: H i Structure in a Blue Compact Dwarf Galaxy. The Astronomical Journal, 142:82, September 2011.

[108] K. A. Herrmann, D. A. Hunter, and B. G. Elmegreen. Surface Brightness Profiles of Dwarf Galaxies. I. Profiles and Statistics. The Astronomical Journal, 146:104, November 2013.

[109] J. Silk. Feedback, Disk Self-Regulation, and Galaxy Formation. The Astrophysical Journal, 481:703-709, May 1997.

[110] M. S. Oey and C. J. Clarke. The superbubble size distribution in the interstellar medium of galaxies. Monthly Notices of the Royal Astronomical Society, 289, August 1997.

[111] A. Gil de Paz, S. Boissier, B. F. Madore, M. Seibert, Y. H. Joe, A. Boselli, T. K. Wyder, D. Thilker, L. Bianchi, S.-C. Rey, R. M. Rich, T. A. Barlow, T. Conrow, K. Forster, P. G. Friedman, D. C. Martin, P. Morrissey, S. G. Neff, D. Schiminovich, T. Small, J. Donas, T. M. Heckman, Y.-W. Lee, B. Milliard, A. S. Szalay, and S. Yi. The GALEX Ultraviolet Atlas of Nearby Galaxies. The Astrophysical Journal, Supplement, 173:185-255, December 2007.

[112] D. A. Thilker, L. Bianchi, G. Meurer, A. Gil de Paz, S. Boissier, B. F. Madore, A. Boselli, A. M. N. Ferguson, J. C. Muñoz-Mateos, G. J. Madsen, S. Hameed, R. A. Overzier, K. Forster, P. G. Friedman, D. C. Martin, P. Morrissey, S. G. Neff, D. Schiminovich, M. Seibert, T. Small, T. K. Wyder, J. Donas, T. M. Heckman, Y.-W. Lee, B. Milliard, R. M. Rich, A. S. Szalay, B. Y. Welsh, and S. K. Yi. A Search for Extended Ultraviolet Disk (XUV-Disk) Galaxies in the Local Universe. The Astrophysical Journal, Supplement, 173:538-571, December 2007. 
[113] J. G. Funes and E. M. Corsini, editors. Formation and Evolution of Galaxy Disks, volume 396 of Astronomical Society of the Pacific Conference Series, October 2008.

[114] C. L. Martin. Properties of outflows from dwarf galaxies: insights into the evolution of the star formation rate. In M. F. Bietenholz, N. Bartel, M. P. Rupen, A. J. Beasley, D. A. Graham, V. I. Altunin, T. Venturi, G. Umana, and J. E. Conway, editors, Bulletin of the American Astronomical Society, volume 29 of Bulletin of the AAS, page 1405, December 1997.

[115] A. J. Youngblood and D. A. Hunter. The Luminosity Functions and Size Distributions of Hir Regions in Irregular Galaxies. The Astrophysical Journal, 519:55-68, July 1999.

[116] B. G. Elmegreen and W.-H. Chiang. Runaway expansion of giant shells driven by radiation pressure from field stars. The Astrophysical Journal, 253:666-678, February 1982.

[117] M. A. Dopita, D. S. Mathewson, and V. L. Ford. Shapley constellation III - A region of self-propagating star formation. The Astrophysical Journal, 297:599606, October 1985. 
VITA

\section{NAU RAJ POKHREL}

2002

B.Sc., Physics

Tribhuvan University

Kathmandu, Nepal

2005

M.Sc., Physics

Tribhuvan University

Kathmandu, Nepal

2010-2016

Ph.D. Candidate, Physics

Florida International University

Miami, Florida

\section{SELECTED PUBLICATIONS AND PRESENTATIONS}

Ashley, T., Elmegreen, B., Johnson, M., Nidever, D., Simpson C., Pokhrel, N., "The H i Chronicles of LITTLE THINGS BCDs II: The Origin of IC 10's H i Structure," The Astronomical Journal, Volume 148, Issue 6, article id. 130, 16 pp. (2014)

Janzen, A., Klopf, E., Lockman, F., Montez Jr, R., Plarre, K., Pokhrel, N., Prestage, R., Selina, R., Togi, A., Zomederis, M., "Limits on the H i Content of the Dwarf Galaxy Hydra II," Astronomy \& Astrophysics, 584, A101 (2015)

Pokhrel, N., Simpson, C., "H I Structure and Kinematics in the LITTLE THINGS Dwarf Galaxies," American Astronomical Society, 227th AAS Meeting, id.111.05, Kissimmee, FL (January 4-8, 2016)

Pokhrel, N., Simpson, C., Bagetakos, I., "H I Porosity and Star Formation in LITTLE THINGS Dwarf Galaxies," Superbubbles, H i holes and Supershells, Munich, Germany (November 10-12, 2014) 